\title{
Designing of metallic photonic structures and applications
}

by

\author{
Yong-Sung Kim
}

A dissertation submitted to the graduate faculty

in partial fulfillment of the requirements for the degree of

DOCTOR OF PHILOSOPHY

Major: Condensed Matter Physics

Program of Study Committee:

Kai-Ming Ho, Major Professor

Rana Biswas

Michael Tringides

H. Bert Crawley

Kristen Constant

Iowa State University

Ames, Iowa

2006 
Graduate College

Iowa State University

This is to certify that the doctoral dissertation of

$$
\text { Yong-Sung Kim }
$$

has met the dissertation requirements of Iowa State University

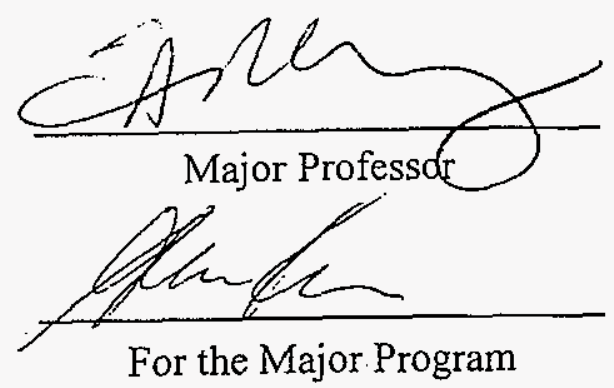




\section{TABLE OF CONTENTS}

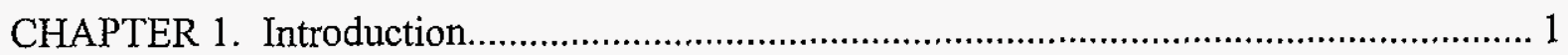

CHAPTER 2. Plane Wave Based Transfer Matrix Method and Modal Expansion Method... 4

2.1 Plane Wave Based Transfer Matrix Method.................................................................... 4

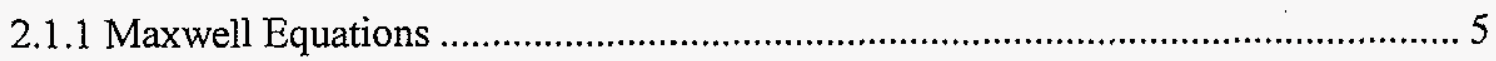

2.1.2 Electro-magnetic Field inside a Grating Slice …….............................................. 6

2.1.3 Electro-magnetic Field around a Grating Slice..................................................... 9

2.1.4 Matching Boundary Conditions ........................................................................... 11

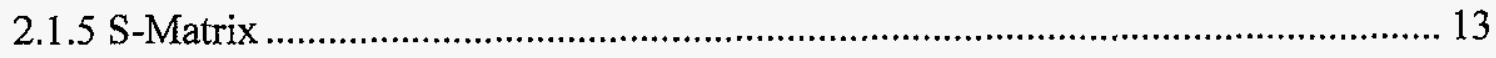

2.1.6 The Total S-Matrix ..................................................................................... 14

2.1.7 Transmission and Reflection Spectra.............................................................. 15

2.1.8 From Wave Scattering to Photonic Band Diagram............................................... 16

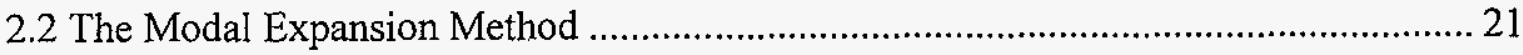

2.2.1 In-Plane Incidence of Plane Waves ................................................................. 22

2.2.2 Off-Plane Conical Incidence of Plane Waves........................................................ 27

2.2.3 Metallic Layer-by-Layer Photonic Crystal Gratings in Mid-Infrared Regimes ... 39

CHAPTER 3. Photonic Band Gaps of Conformally Coated Structures .................................. 53

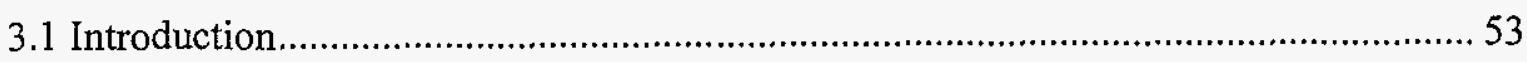

3.2 Coated Structures.................................................................................................... 55

3.3 Metallic Coating - Theoretical and Experimental Results .......................................... 56 
3.4 Dielectric Coating - Theoretical and Experimental Results.................................... 58

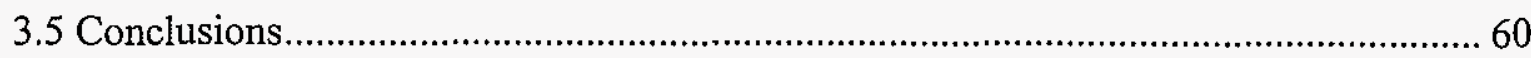

CHAPTER 4. Thermal Emission from Metallic Photonic Crystals .................................... 68

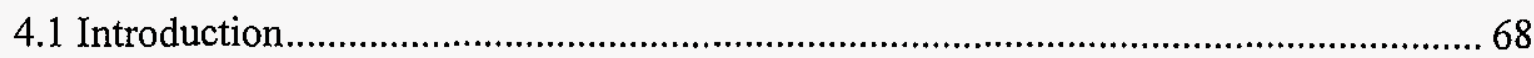

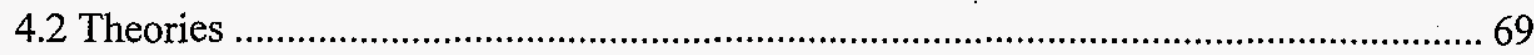

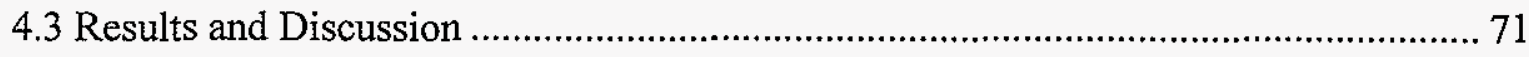

4.4 Summary and Conclusion ........................................................................... 74

CHAPTER 5. Analysis of Bulb System Enclosed by Photonic Structures ......................... 83

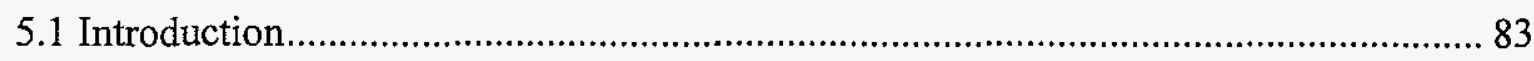

5.2 Theories of a blackbody and the role of photonic structures ................................... 84

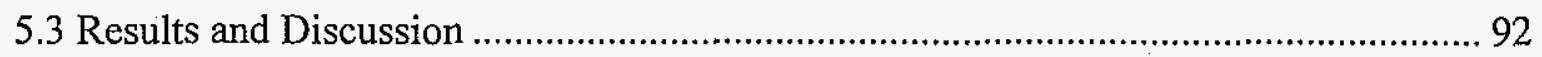

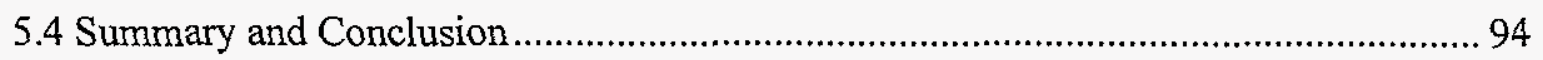

Appendix 1. Reflected or Absorbed Beam by a Photonic Structure ............................... 95

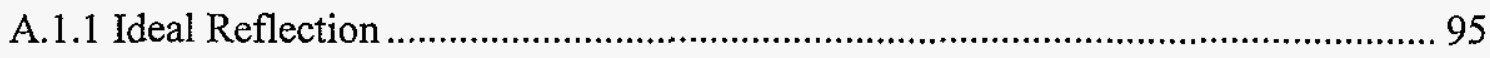

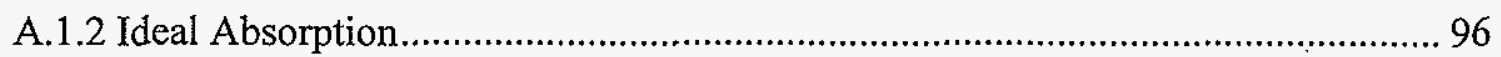

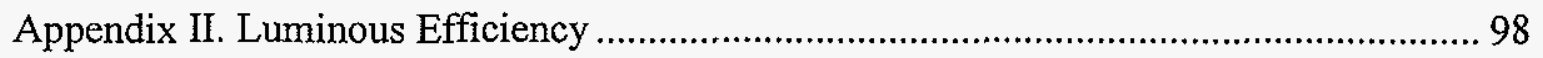

CHAPTER 6. General Conclusion ........................................................................... 106

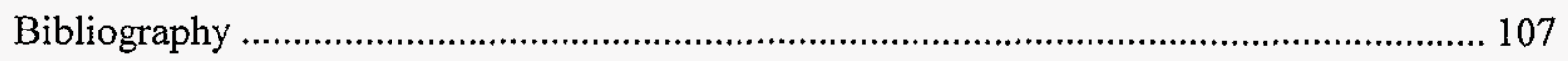




\section{CHAPTER 1. Introduction}

Photonic crystals or photonic band gap materials are periodic dielectric structures in one, two or three dimensions, and they have many interesting effects on the propagation of light inside them. Typically photonic crystals are composed of two different dielectric materials, for example air and silicon, arranged in a periodic lattice structure. An important figure of merit for such a construction is the index contrast of these two dielectric materials, which is the difference of their refractive indexes. The refractive index of a dielectric material is the square root of its relative permeability.

This has been an active research area since around 1987. Yablonovitch [1], John [2], and Ho [3] produced some of the first publications concerning photonic crystal. These people are considered to have been the first to suggest the idea of a photonic crystal.

In one dimension, however, periodic dielectric structures were being investigated long before these periodic structures were named photonic crystal. The conventional dielectric multilayer or Bragg reflector consists of alternating layers of two different dielectric materials. The interfaces between layers act as scatterers for the incident beam on the stack. As is well known from any standard optics text certain frequencies incident normal to the layers are transmitted through the stack while others are forbidden to propagate and are completely reflected. The band structure for a typical Bragg reflector divides into allowed bands where at a given frequency there are real wavevectors (k) corresponding to modes 
which propagate through the system and band gaps where no real k's exist. In the band gap all k's are complex and correspond to evanescent waves which decay into the crystal. These modes cannot be excited in an infinite photonic crystal, but if the translational symmetry is broken by a surface or a defect, then it may be possible to create a localized surface or defect mode within the band gap.

The Bragg reflector is a very useful object and has found many applications; as a stop-band filter, as a virtually loss-free mirror or, by introducing a defect layer, as a cavity for the distributed feedback laser.

However, the Bragg reflector has one major limitation - band gaps only appear for waves traveling at normal incidence. As we move away from normal incidence the gaps quickly disappear. Ideally, a photonic crystal has a complete band gap for which propagation is forbidden in all directions. $\mathrm{T}$ his is the optical analog of a semiconductor that has a complete band gap between the valence and conduction bands. A photonic crystal with a complete band gap would find use in all the applications of the Bragg reflector. Moreover, such a crystal would radically alter the spontaneous emission properties of an excited atom placed within it. The atom would be unable to emit a photon into the band gap and so the atom and photon must form a new kind of bound state. Making a defect in the photonic lattice could create a single defect mode in the gap. The atom could emit into that mode and no other so, if we arrange things properly, we could make a highly efficient laser cavity by completely inhibiting radiation into any mode other than the lasing one.

Photonic band gaps can be used in many ways. For example a line defect in a two- or three-dimensional photonic crystal can act as a waveguide, forcing certain frequencies to 
propagate only along the line. The surface of a photonic crystal can act as perfect mirror for the frequencies within the gap.

It has also been suggested that a metallic photonic crystal(MPC) structure may modify a thermal emission spectrum[4,5]. The experiment was carried out by Lin $e t$ al. using a three dimensional woodpile structure made of tungsten[6]. The resulting thermal emission was shown to be suppressed in the photonic band gap and enhanced near the band edge. Promising experimental data have demonstrated that these metallic microstructures can be useful for incandescent lamp applications and for thermal photovoltaic power generation. 


\section{CHAPTER 2. Plane Wave Based Transfer Matrix Method and Modal Expansion}

\section{Method}

The photonic band diagram, a plot of wave vector versus mode frequency of the mode is a basic tool to understand the characteristic of a photonic crystal. A frequency range in the photonic band diagram in which no photons exist in any direction and polarization is called a complete photonic band gap. In this chapter we discuss the basic concept of planewave transfer matrix method which is effectively applicable to dielectric structures and modal expansion method which is effectively applicable to metallic structures.

For the discussion in this chapter we are referring to Lan-Lan Lin, Zhi-Yuan Li, and Kai-Ming Ho [7] and Zhi-Yuan Li and Kai-Ming Ho [8].

\subsection{Plane Wave Based Transfer Matrix Method}

Many theoretical approaches have been developed to calculate the photonic band diagram for two-dimensional (2D) and three-dimensional (3D) photonic crystals. These include conventional plane-wave expansion method (PWM) $[3,10,11]$, real-space transfermatrix method (TMM)[11,12], finite-difference time-domain method [13], and KorringaKohn-Rostoker (KKR) method $[14,15]$. In the conventional PWM, the photonic crystal is considered as an infinite lattice along all directions and Maxwell's equations are solved in a 
way that EM fields are represented by functions quasi-periodic in the whole space. In the real-space TMM and the KKR method, the photonic crystal is regarded as an infinite stack of identical periodic crystal layers along a certain direction. Maxwell's equations are solved within each layer, and the electro-magnetic fields of different layers are connected to each other through a transfer-matrix formulation. In addition to solutions of photonic band structures, the TMM can also calculate the wave scattering by a photonic crystal slab, leading to the transmission and reflection spectra that can be directly observed experimentally.

In this chapter, we will introduce in detail the plane-wave based TMM which is applied to calculate the photonic band diagram and the spectra of photonic crystal structures. The scattering-matrix (S-matrix) algorithm will be also used to avoid numerical instability.

\subsubsection{Maxwell Equations}

We start from Maxwell's equations:

$$
\nabla \times E(r)=i k_{0} H(r), \quad \nabla \times H(r)=-i k_{0} \varepsilon(r) E(r)
$$

Here $\varepsilon(r)$ is the periodic dielectric function of the grating slice, it is homogeneous along the $z$-axis direction. We can rewrite Eq. (2.1) into six partial-differential equations satisfied by $\left(E_{x}, E_{y}, E_{z}\right)$ and $\left(H_{x}, H_{y}, H_{z}\right)$. The $z$-components of EM fields $E_{z}$ and $H_{z}$ can be deleted from these six equations, leading to the following four coupled equations: 


$$
\begin{gathered}
\frac{\partial}{\partial z} E_{x}=\frac{1}{-i k_{0}} \frac{\partial}{\partial x}\left[\frac{1}{\varepsilon}\left(\frac{\partial}{\partial x} H_{y}-\frac{\partial}{\partial y} H_{x}\right)\right]+i k_{0} H_{y} \\
\frac{\partial}{\partial z} E_{y}=\frac{1}{-i k_{0}} \frac{\partial}{\partial y}\left[\frac{1}{\varepsilon}\left(\frac{\partial}{\partial x} H_{y}-\frac{\partial}{\partial y} H_{x}\right)\right]-i k_{0} H_{x} \\
\frac{\partial}{\partial z} H_{x}=\frac{1}{-i k_{0}} \frac{\partial}{\partial x}\left(\frac{\partial}{\partial x} E_{y}-\frac{\partial}{\partial y} E_{x}\right)-i k_{0} \varepsilon E_{y} \\
\frac{\partial}{\partial z} H_{y}=\frac{1}{-i k_{0}} \frac{\partial}{\partial y}\left(\frac{\partial}{\partial x} E_{y}-\frac{\partial}{\partial y} E_{x}\right)+i k_{0} \varepsilon E_{x}
\end{gathered}
$$

\subsubsection{Electro-magnetic Field inside a Grating Slice}

We can write down the plane-wave expansion expressions of the EM fields and the dielectric function,

$$
\begin{gathered}
E(r)=\sum_{i, j} E_{i j}(z) e^{i\left(k_{i, x} x+k_{i, y}, y\right)} \\
H(r)=\sum_{i, j} H_{i j}(z) e^{i\left(k_{y, x} x+k_{i, y} y\right)} \\
\varepsilon(r)=\sum_{i j} \varepsilon_{i j} e^{i \bar{G}_{i j} \bullet \bar{r}} \\
\frac{1}{\varepsilon(r)}=\sum_{i j} \varepsilon_{i j}^{-1} e^{i \bar{G}_{i,} \bullet \vec{r}}
\end{gathered}
$$

Substituting them into Eqs. (2.2)-(2.5) yields 


$$
\begin{gathered}
\frac{\partial E_{i j, x}}{\partial z}=-\frac{i k_{i j, x}}{k_{0}} \sum_{m n} \varepsilon_{i-m, j-n}^{-1}\left(H_{m n, y} k_{m n \cdot x}-H_{m n, x} k_{m n, y}\right)+i k_{0} H_{i j, y} \\
\frac{\partial E_{i j, y}}{\partial z}=-\frac{i k_{i j, y}}{k_{0}} \sum_{m n} \varepsilon_{i-n, j-n}^{-1}\left(H_{m n, y} k_{m n . y}-H_{m n, x} k_{m n, x}\right)-i k_{0} H_{i j, x} \\
\frac{\partial H_{i j, x}}{\partial z}=\frac{i k_{i j, x}}{k_{0}} \sum_{m n} \delta_{i m, j n}\left(E_{m n . y} k_{m n, x}-E_{m n, x} k_{m n, y}\right)-i k_{0} \sum_{m n} \varepsilon_{i-m, j-n} E_{m n \cdot y} \\
\frac{\partial H_{i j, y}}{\partial z}=\frac{i k_{i j, y}}{k_{0}} \sum_{m n} \delta_{i m, j n}\left(E_{m n ., y} k_{m n, x}-E_{m n, x} k_{m n, y}\right)+i k_{0} \sum_{m n} \varepsilon_{i-m, j-n} E_{m n . x}
\end{gathered}
$$

Now define column vectors

$$
E=\left(\begin{array}{c}
\vdots \\
E_{i j, x} \\
E_{i j, y} \\
\vdots
\end{array}\right), H=\left(\begin{array}{c}
\vdots \\
H_{i j, x} \\
H_{i j, y} \\
\vdots
\end{array}\right)
$$

Eqs. (2.10)-(2.13) can be written in a concise matrix form

$$
\frac{\partial}{\partial z} E=T_{1} H, \frac{\partial}{\partial z} H=T_{2} E
$$

where the matrices $T_{1}$ and $T_{2}$ are defined as 


$$
\begin{array}{r}
T_{1}^{i j ; m n}=\frac{i}{k_{0}}\left(\begin{array}{lr}
k_{i j, x} \varepsilon_{i j ; m n}^{-1} k_{n m, y} & -k_{i j, x} \varepsilon_{i j ; m n}^{-1} k_{n m, x}+k_{0}^{2} \delta_{i j ; m n} \\
k_{i j, y} \varepsilon_{i j ; m n}^{-1} k_{n m, y}-k_{0}^{2} \delta_{i j ; m n} & -k_{i j, y} \varepsilon_{i j ; m n}^{-1} k_{m n, x}
\end{array}\right) \\
T_{2}^{i j ; m n}=\frac{i}{k_{0}}\left(\begin{array}{lr}
-k_{i j, x} \delta_{i j ; m n} k_{n m, y} & k_{i j, x} \delta_{i j ; m n} k_{n m, x}-k_{0}^{2} \varepsilon_{i j ; m n} \\
-k_{i j, y} \delta_{i j ; m n} k_{n m, y}+k_{0}^{2} \varepsilon_{i j ; m n} & k_{i j, y} \delta_{i j ; m n} k_{m n, x}
\end{array}\right)
\end{array}
$$

From Eq. (2.14) we finally obtain an Eigenvalue problem for the electric field,

$$
\frac{\partial^{2}}{\partial z^{2}} E=\left(T_{1} T_{2}\right) E=P E
$$

Now suppose we have used $N_{0}$ plane waves in the expansion, then $T_{1}, T_{2}$ and $P$ are all $\left(2 N_{0}\right) \times\left(2 N_{0}\right)$ matrices. Solution of Eq. (2.15) will give us $2 N_{0}$ eigenvalues (denoted as $\beta_{i}{ }^{2}$, $i=1,2, \cdots, 2 N_{0}$, with $\left.\operatorname{Im}\left(\beta_{i}\right) \geq 0\right)$ of the matrix $-P=-T_{1} \cdot T_{2}$. In addition, the $\left(2 N_{0}\right) \times\left(2 N_{0}\right)$ matrix $S_{a}$, whose $j_{t h}$ column is the eigenvector corresponding to the eigenvalue $\beta_{i}{ }^{2}$, can also be obtained simultaneously. The eigenmode corresponding to $\beta_{i}{ }^{2}$ is ${ }^{\prime} E_{i}(z)=E_{a, i}^{+}(z)+\dot{E}_{a, i}^{-}(z)$, $E_{i}^{+}(z)=E_{a, i}^{+} e^{i \dot{\beta}_{i}\left(z-z_{i-1}\right)}, E_{i}^{-}(z)=E_{a, i}^{-} e^{-i \beta_{i}\left(z-z_{i-1}\right)}$, where $E_{i}^{+}$and $E_{i}^{-}$are both unknown variables. Further define column vector $\beta=\left(\cdots, \beta_{i}, \cdots\right)^{T} \quad, \quad E_{a}^{+}=\left(\cdots, E_{a, i}^{+}(z), \cdots\right)^{T} \quad$ and $E_{a}^{-}=\left(\cdots, E_{a, i}^{-}(z), \cdots\right)^{T}$. The electric field column vector $\mathrm{E}$ is now expressed into the superposition of all the eigenmodes, $E=S_{a}\left(E_{a}^{+}+E_{a}^{-}\right)$. The corresponding magnetic field column vector are obtained from Eq. (2.14) and we get $H=T_{1}^{-1} \frac{\partial}{\partial z} E=T_{1}^{-1} S_{a} \frac{\partial}{\partial z}\left(E_{a}^{+}+E_{a}^{-}\right)=i T_{1}^{-1} S_{a} \beta\left(E_{a}^{+}-E_{a}^{-}\right)=T_{a}\left(E_{a}^{+}-E_{a}^{-}\right)$, where $T_{a}=i T_{1}^{-1} S_{a} \beta$ 
It is convenient to express the electric and magnetic fields at an arbitrary point inside the grating slice into a concise form:

$$
\left(\begin{array}{l}
E(z) \\
H(z)
\end{array}\right)=\left(\begin{array}{cc}
S_{a} & S_{a} \\
T_{a} & -T_{a}
\end{array}\right)\left(\begin{array}{c}
E_{a}^{+} \\
E_{a}^{-}
\end{array}\right)
$$

\subsubsection{Electro-magnetic Field around a Grating Slice}

Having expressed the EM fields inside the slice, we need to further solve the EM fields around the slice. The EM fields in the two air films around the grating slice can also be solved in the same way, The EM fields in the two air thin films around the $i_{t h}$ slice is both consisting of forwards and backwards propagating plane waves. The tangential components of the electric field in the left hand-side air film can be written into

$$
E_{x(y)}(r)=\sum_{i j} E_{i j, x(y)}(z) e^{i k_{j, x} x+i k_{y, y} y}=\sum_{i j}\left[E_{i j, x(y)}^{+}(z)+E_{i j, x(y)}^{-}(z)\right] e^{i k_{i, x} x+i k_{i, y} y}
$$

where $E_{i j, x(y)}^{+}(z)=E_{i j, x(y)}^{+} e^{i \beta_{y}\left(z-z_{i-1}\right)}=E_{i j, x(y)}^{+}, E_{i j, x(y)}^{-}(z)=E_{i j, x(y)}^{-} e^{-i \beta_{y}\left(z-z_{i-1}\right)}=E_{i j, x(y)}^{-}$, since $z=z_{i-1}$.

$\beta_{i j}$ is given by $\beta_{i j}=\left(k_{0}^{2}-k_{i j, x}^{2}-k_{i, y}^{2}\right)$ for $k_{0}^{2}-k_{i j, x}^{2}-k_{i j, y}^{2} \geq 0$, and $\beta_{i j}=i\left(k_{i j, x}^{2}+k_{i j, y}^{2}-k_{0}^{2}\right)$ for $k_{0}^{2}-k_{i j, x}^{2}-k_{i j, y}^{2}<0$.

The tangential components of the magnetic field are

$$
H_{x(y)}(r)=\sum_{i j} H_{i j, x(y)}(z) e^{i k_{y, x} x+i k_{y, y} y}=\sum_{i j}\left[H_{i j, x(y)}^{+}(z)+H_{i j, x(y)}^{-}(z)\right] e^{i k_{j, x} x+i k_{j, y} y}
$$


where $H_{i j, x(y)}^{+}(z)=H_{i j, x(y)}^{+} e^{i \beta_{i j}\left(z-z_{i-1}\right)}=H_{i j, x(y)}^{+}, H_{i j, x(y)}^{-}(z)=H_{i j, x(y)}^{-} e^{-i \beta_{y}\left(z-z_{i-1}\right)}=H_{i j, x(y)}^{-}$. For each Bragg wave vector we have the following relation between the $\mathrm{H}$ and $\mathrm{E}$ fields: $\left(H_{i j, x}^{+}, H_{i j, y}^{+}\right)^{T}=T_{0, i j}\left(E_{i j, x}^{+}, E_{i j, y}^{+}\right)^{T}$ and $\left(H_{i j, x}^{-}, H_{i j, y}^{-}\right)^{T}=-T_{0, i j}\left(E_{i j, x}^{-}, E_{i j, y}^{-}\right)^{T}$, where the superscript " $T$ " denotes matrix transposition. The $2 \times 2$ matrix $T_{0, i j}$ has matrix elements $T_{0, i j}^{11}=-k_{i j, x} k_{i j, y} /\left(k_{0} \beta_{i j}\right) \quad, \quad T_{0, i j}^{12}=\left(k_{i j, x}^{2}-k_{0}^{2}\right) /\left(k_{0} \beta_{i j}\right) \quad, \quad T_{0, j j}^{21}=\left(k_{0}^{2}-k_{i j, x}^{2}\right) /\left(k_{0} \beta_{i j}\right) \quad, \quad$ and $T_{0, i j}^{22}=k_{i j, x} k_{i j, y} /\left(k_{0} \beta_{i j}\right)$. The EM fields in the right hand-side air film have the same form of expansion. In Eqqs. (2.17) and (2.18) we simply replace $E_{i j, x(y)}^{ \pm}$and $H_{i j, x(y)}^{ \pm}$with $U_{i j, x(y)}^{ \pm}$and $V_{i j, x(y)}^{ \pm}$, respectively. $V_{i j, x(y)}^{ \pm}$and $U_{i j, x(y)}^{ \pm}$are also connected each other through the $2 \times 2$ matrix $T_{0, i j}$

By defining column vectors $\Omega_{i-1}^{ \pm}=\left(\cdots, E_{i j, x}^{ \pm}, E_{i j, y}^{ \pm}, \cdots\right)^{T}, \Omega_{i}^{ \pm}=\left(\cdots, U_{i j, x}^{ \pm}, U_{i j, y}^{ \pm}, \cdots\right)^{T}$, where $-N \leq i \leq N,-M \leq j \leq M$, with $N$ and $M$ being the truncation orders, we have

$$
\left(\begin{array}{l}
\Omega_{i}^{+} \\
\Omega_{i}^{-}
\end{array}\right)=T_{i}\left(\begin{array}{l}
\dot{\Omega}_{i-1}^{+} \\
\Omega_{i-1}^{-}
\end{array}\right)=\left(\begin{array}{cc}
t_{11}^{i} & t_{12}^{i} \\
t_{21}^{i} & t_{22}^{i}
\end{array}\right)\left(\begin{array}{l}
\Omega_{i-1}^{+} \\
\Omega_{i-1}^{-}
\end{array}\right)
$$

$T_{i}$ is called the transfer matrix for the $i_{t h}$ slice.

It is easy to find that the fields in the transmission region $\Omega_{n}^{ \pm}$are related to the fields in the incident region $\Omega_{0}^{ \pm}$by the total transfer matrix of the slab,

$$
\left(\begin{array}{l}
\Omega_{n}^{+} \\
\Omega_{n}^{-}
\end{array}\right)=T\left(\begin{array}{l}
\Omega_{0}^{+} \\
\Omega_{0}^{-}
\end{array}\right)
$$


where $T=T_{n} T_{n-1} \cdots T_{2} T_{1}$. Notice that $\Omega_{0}^{+}=E_{0}=\left(\cdots, E_{0, x}, E_{0, y}, \cdots\right)^{T} \quad, \quad$ and $\Omega_{0}^{-}=E_{r}=\left(\cdots, E_{i j, x}^{r}, E_{i j, y}^{r}, \cdots\right)^{T}$ are column vectors for the incident and reflection waves, $\Omega_{n}^{+}=E_{t}=\left(\cdots, E_{i j, x}^{t}, E_{i j, y}^{t}, \cdots\right)^{T}$ is for the transmission waves, while $\Omega_{n}^{-}=0$ since no backwards propagating waves exist in the transmission region.

\subsubsection{Matching Boundary Conditions}

We can rewrite electro-magnetic fields around the grating slice in a way similar to Eq. (2.16). Match of boundary conditions requests all tangential field components of each plane wave be continuous at the two interfaces between the air films and the grating slice. At the left interface $z=z_{i-1}$,

$$
\left(\begin{array}{cc}
S_{0} & S_{0} \\
T_{0} & -T_{0}
\end{array}\right)\left(\begin{array}{c}
\Omega_{i-1}^{+} \\
\Omega_{i-1}^{-}
\end{array}\right)=\left(\begin{array}{cc}
S_{a} & S_{a} \\
T_{a} & -T_{a}
\end{array}\right)\left(\begin{array}{c}
E_{a}^{+}\left(z_{i-1}\right) \\
E_{a}^{-}\left(z_{i-1}\right)
\end{array}\right)
$$

while at the right interface $z=z_{i}$,

$$
\left(\begin{array}{cc}
S_{0} & S_{0} \\
T_{0} & -T_{0}
\end{array}\right)\left(\begin{array}{l}
\Omega_{i}^{+} \\
\Omega_{i}^{-}
\end{array}\right)=\left(\begin{array}{cc}
S_{a} & S_{a} \\
T_{a} & -T_{a}
\end{array}\right)\left(\begin{array}{l}
E_{a}^{+}\left(z_{i}\right) \\
E_{a}^{-}\left(z_{i}\right)
\end{array}\right)
$$

where $S_{0}$ and $T_{0}$ are counterparts of $S_{a}$ and $T_{a}$ in an air film. They can be analytically solved. Actually $S_{0}=I$, a unit matrix, $T_{0}$ is a block-diagonal matrix each block of which is a $2 \times 2$ matrix already given by $T_{0, i j}$ in Eq. (2.4). In the grating slice, 


$$
\left(\begin{array}{l}
E_{a}^{+}\left(z_{i}\right) \\
E_{a}^{-}\left(z_{i}\right)
\end{array}\right)=\left(\begin{array}{cc}
e^{i \beta h} & 0 \\
0 & e^{-i \beta h}
\end{array}\right)\left(\begin{array}{l}
E_{a}^{+}\left(z_{i-1}\right) \\
E_{a}^{-}\left(z_{i-1}\right)
\end{array}\right)
$$

where $h=z_{i}-z_{i-1}$ is the thickness of the slice, $e^{i \beta h}$ denotes a $\left(2 N_{0}\right) \times\left(2 N_{0}\right)$ diagonal matrix whose element is $e^{i \beta_{i} h}, i=1,2, \cdots, 2 N_{0}$. Deleting $\left[E_{a}^{+}\left(z_{i-1}\right), E_{a}^{-}\left(z_{i-1}\right)\right]^{T}$ and $\left[E_{a}^{+}\left(z_{i}\right), E_{a}^{-}\left(z_{i}\right)\right]^{T}$ from Eqs. (2.21)-(2.23) and making some analytical derivations yields

$$
\left(\begin{array}{l}
\Omega_{i}^{+} \\
\Omega_{i}^{-}
\end{array}\right)=\left(\begin{array}{ll}
a_{11} & a_{12} \\
a_{21} & a_{22}
\end{array}\right)^{-1}\left(\begin{array}{cc}
e^{i \beta h} & 0 \\
0 & e^{-i \beta h}
\end{array}\right)\left(\begin{array}{ll}
a_{11} & a_{12} \\
a_{21} & a_{22}
\end{array}\right)\left(\begin{array}{l}
\Omega_{i-1}^{+} \\
\Omega_{i-1}^{-}
\end{array}\right)
$$

where $a_{11}=\frac{1}{2}\left(S_{a}^{-1} S_{0}+T_{a}^{-1} T_{0}\right), a_{12}=\frac{1}{2}\left(S_{a}^{-1} S_{0}-T_{a}^{-1} T_{0}\right)$, and $a_{21}=a_{12}, a_{22}=a_{11}$. Eq. (2.22) has already present us the $T$-matrix for slice $i$, namely,

$$
T_{i}=\left(\begin{array}{ll}
t_{11}^{i} & t_{12}^{i} \\
t_{21}^{i} & t_{22}^{i}
\end{array}\right)=\left(\begin{array}{ll}
a_{11} & a_{12} \\
a_{21} & a_{22}
\end{array}\right)^{-1}\left(\begin{array}{cc}
e^{i \beta h} & 0 \\
0 & e^{-i \beta h}
\end{array}\right)\left(\begin{array}{ll}
a_{11} & a_{12} \\
a_{21} & a_{22}
\end{array}\right)
$$

Now it becomes clear why the $T$-matrix formalism is numerically unstable for thick gratings. Look at the two phase factors $e^{i \beta h}$ and $e^{-i \beta h}$. For those Bragg wave components where $\beta_{i}$ has a significant imaginary part, one of them must be exponentially increasing through the whole slab of gratings, rendering numerical instability such as overflow. 


\subsubsection{S-Matrix}

One method to overcome this numerical instability is the so-called S-matrix method [16]. In this method, the transfer matrix for the $i_{t h}$ slice in Eq. (2.19) is redefined in another way as follows:

$$
\left(\begin{array}{c}
\Omega_{i}^{+} \\
\Omega_{i-1}^{-}
\end{array}\right)=s_{i}\left(\begin{array}{c}
\Omega_{i-1}^{+} \\
\Omega_{i}^{-}
\end{array}\right)=\left(\begin{array}{cc}
s_{11}^{i} & s_{12}^{i} \\
s_{21}^{i} & s_{22}^{i}
\end{array}\right)\left(\begin{array}{c}
\Omega_{i-1}^{+} \\
\Omega_{i}^{-}
\end{array}\right)
$$

$s^{i}$ is called the $S$-matrix for slice $i . s^{i}$ is related with $T_{i}$ as follows: $s_{11}^{i}=t_{11}^{i}-t_{12}^{i}\left[t_{22}^{i}\right]^{-1} t_{21}^{i}$, $s_{12}^{i}=t_{12}^{i}\left[t_{22}^{i}\right]^{-1}, s_{21}^{i}=-\left[t_{22}^{i}\right]^{-1} t_{21}^{i}, s_{22}^{i}=\left[t_{22}^{i}\right]^{-1}$. In principle, the $S$-matrix can be calculated from the $T$-matrix through a transformation. However, because the $T$-matrix itself is consisting of very large numbers $e^{-i \beta h}$ and very small numbers $e^{i \beta h}$, any transformation through numerical manipulations [such as a matrix inverse] on this $T$-matrix will generate problems of numerical errors, especially when the plane wave number is large. Therefore, we prefer to derive the $S$-matrix for slice $i$ directly from Eq. (2.24) without the aid of the $T$-matrix, largely depending on analytical derivation. This proves to be more stable and reliable numerically. After some algebraic manipulations, we finally arrive at

$$
s^{i}=\left(\begin{array}{ll}
s_{11}^{i} & s_{12}^{i} \\
s_{21}^{i} & s_{22}^{i}
\end{array}\right)=\left(\begin{array}{cc}
p_{1} t_{1}+p_{2} t_{2} & p_{1} t_{2}+p_{2} t_{1} \\
p_{1} t_{2}+p_{2} t_{1} & p_{1} t_{1}+p_{2} t_{2}
\end{array}\right)
$$


where $p_{1}=\left[a_{11}-e^{i \beta h} a_{12} a_{11}^{-1} e^{i \beta h} a_{12}\right]^{-1}, p_{2}=a_{11}^{-1} e^{i \beta h} a_{12}\left[a_{11}-e^{i \beta h} a_{12} a_{11}^{-1} e^{i \beta h} a_{12}\right]^{-1}, t_{1}=e^{i \beta h} a_{11}$, and $t_{2}=-a_{12}$. We now see that in the inverse of a matrix, where a numerical calculation is necessary, no very large or very small matrix elements are involved. From Eq. (2.27), it is obvious that $s^{i}$ is a block-symmetric matrix, reflecting the fact that the grating slice is symmetric with respect to its middle plane at $z=\left(z_{i-1}+z_{i}\right) / 2$.

\subsubsection{The Total S-Matrix}

The total $S$-matrix of the whole slab is connected to individual $s_{i}$ through an iteration algorithm. Suppose the total $S$-matrix for the first $n-1$ slices and the $S$-matrix for slice $n$ have been calculated to be $S^{n-1}$ and $s^{n}$, respectively, it can be shown that the total $S$-matrix for the first $n$ slices $S^{n}$ is given by

$$
\begin{gathered}
S_{11}^{n}=s_{11}^{n}\left[I-S_{12}^{n-1} S_{21}^{n}\right]^{-1} S_{11}^{n-1} \\
S_{12}^{n}=s_{12}^{n}+s_{11}^{n} S_{12}^{n-1}\left[I-S_{21}^{n} S_{12}^{n-1}\right]^{-1} s_{22}^{n-1} \\
S_{21}^{n}=S_{21}^{n}+S_{22}^{n-1} s_{21}^{n}\left[I-S_{12}^{n-1} s_{21}^{n}\right]^{-1} S_{11}^{n-1} \\
S_{22}^{n}=S_{22}^{n-1}\left[I-s_{21}^{n} S_{12}^{n-1}\right]^{-1} s_{22}^{n}
\end{gathered}
$$

where $I$ is an unit matrix. Then fields in the transmission and reflection waves are connected to the incident waves through

$$
\left(\begin{array}{l}
\Omega_{n}^{+} \\
\Omega_{0}^{-}
\end{array}\right)=S^{n}\left(\begin{array}{l}
\Omega_{0}^{+} \\
\Omega_{n}^{-}
\end{array}\right)
$$


from which we get

$$
E_{t}=S_{11}^{n} E_{0}, \quad E_{r}=S_{21}^{n} E_{0}
$$

In the calculation of the total $S$-matrix for a slab, we can first set $S^{0}=I$, which corresponds to the $S$-matrix for an air slice. We then solve the $S$-matrix for slice $1 s^{1}$, and use the iteration algorithm Eqs. (2.28)-(2.31) to obtain the total $S$-matrix $S^{1}$ for the first one slice. Repeat the procedure, we can finally obtain the total $S$-matrix for the whole slab.

\subsubsection{Transmission and Reflection Spectra}

After we have obtained coefficients for the reflection and transmission waves, the transmission and reflection spectra are calculated by

$$
T=\sum_{i j} T_{i j}=\sum_{i j} \frac{\left|\mathbf{E}_{i j}^{t}\right|^{2}\left|\beta_{i j}\right|}{\left|\mathbf{E}_{0}\right|^{2}\left|k_{0 z}\right|}
$$

and

$$
R=\sum_{i j} R_{i j}=\sum_{i j} \frac{\left|\mathbf{E}_{i j}^{r}\right|^{2}\left|\beta_{i j}\right|}{\left|\mathbf{E}_{0}\right|^{2}\left|k_{0 z}\right|}
$$

where the summation is run over those homogeneous Bragg waves with lateral wave vectors $k_{i j, x}^{2}+k_{i j, y}^{2} \leq k_{0}^{2} . \mathbf{E}_{i j}^{t}$ and $\mathbf{E}_{i j}^{r}$ are the amplitudes of the transmission and reflection Bragg wave in the $(i j)_{t h}$ order. 


\subsubsection{From Wave Scattering to Photonic Band Diagram}

With the layer transfer-matrix at hand, we can directly solve the photonic band structure. Assume the primitive lattice vectors of a 3D photonic crystal are $\mathbf{a}_{1}, \mathbf{a}_{2}$, and $\mathbf{a}_{3}$. Note that these three vectors are not necessarily orthogonal to each other for a general lattice, say, a face-centered cubic (FCC) or face-centered tetragonal (FCT) lattice. We can always set the plane formed by $\mathbf{a}_{1}$ and $\mathbf{a}_{2}$ as the $X O Y$ plane, which means that in this plane we have a 2D lattice with primitive lattice vectors $\mathbf{a}_{1}$ and $\mathbf{a}_{2}$. The corresponding reciprocal lattice vectors are $\mathbf{b}_{1}=2 \pi\left(\mathbf{a}_{2} \times \hat{z}\right) /\left[\left(\mathbf{a}_{1} \times \mathbf{a}_{2}\right) \cdot \hat{z}\right], \quad \mathbf{b}_{2}=2 \pi\left(\hat{z} \times \mathbf{a}_{1}\right) /\left[\left(\mathbf{a}_{1} \times \mathbf{a}_{2}\right) \cdot \hat{z}\right]$, and a general reciprocal vector is given by $\mathbf{G}_{i j}=i \mathbf{b}_{1}+j \mathbf{b}_{2}$. The whole infinite photonic crystal is looked upon as an infinite number of layers stacking along the $z$-axis direction, every layer having the same primitive lattice vectors $\left(\mathbf{a}_{1}\right.$ and $\left.\mathbf{a}_{2}\right)$ and reciprocal lattice vectors $\left(\mathbf{b}_{1}\right.$ and $\left.\mathbf{b}_{2}\right)$. The key from a general wave scattering problem to a photonic band structure problem is to impose a periodic boundary condition along the stacking direction of the infinite grating. According to Bloch's theorem, the field at $\mathbf{r}$ is connected to the field at $\mathbf{r}+\mathbf{R}$ through $u(\mathbf{r}+\mathbf{R})=e^{i k \cdot \mathbf{R}} u(\mathbf{r})$, where $u$ is one of the components of either E-field or H-field, $\mathbf{k}=\left(k_{x}, k_{y}, k_{z}\right)$ is the Bloch's wave vector, and $\mathbf{R}$ is a lattice vector of the $3 \mathrm{D}$ lattice. The periodic boundary condition along the $z$-axis direction leads to

$$
u\left(\mathbf{r}+\mathbf{a}_{3}\right)=e^{i \mathbf{k} \cdot \mathbf{a}_{3}} u(\mathbf{r})=\exp \left[i\left(k_{x} a_{3, x}+k_{y} a_{3, y}+k_{z} a_{3, z}\right)\right] u(\mathbf{r})
$$


We need to work out the transfer matrix connecting the field at $\mathbf{r}$ and $\mathbf{r}+\mathbf{a}_{3}$. The transfer-matrix is strictly propagating along the stacking direction, namely, the $z$-axis direction. Therefore, after we get the transfer-matrix connecting $u\left(x, y, z_{0}\right)$ and $u\left(x, y, z_{0}+a_{3, z}\right)$, we should further phase shift $u\left(x, y, z_{0}+a_{3, z}\right)$ to $u\left(x+a_{3, x}, y+a_{3, y}, z_{0}+a_{3, z}\right)$.

Observing that $u\left(x, y, z_{0}+a_{3, z}\right)=\sum u_{i j}\left(z_{0}+a_{3, z}\right) \exp \left[i k_{i j, x} x+i k_{i, y} y\right] \quad, \quad$ and $u\left(x+a_{3, x}, y+a_{3, y}, z_{0}+a_{3, z}\right)=\sum u_{i j}\left(z_{0}+a_{3, z}\right) \exp \left[i k_{i j, x} a_{3, x}+i k_{i j, y} a_{3, y}\right] \exp \left[i k_{i j, x} x+i k_{i j, y} y\right]$, we can easily find out the transformation rule of both the $T$-matrix and $S$-matrix under this phase shift. Another important point is that in application of the transfer-matrix technique, the Bragg wave vectors for the 2D lattice in the $X O Y$ plane should be $\left(k_{i j, x}, k_{i j, y}\right)=\left(k_{x}+G_{i j, x}, k_{y}+G_{i j, y}\right)$.

After the transfer-matrix connecting $u\left(\mathbf{r}+\mathbf{a}_{3}\right)$ and $u(\mathbf{r})$ is finally stabilized, we are ready to move forward to solve the photonic band structure. Denoting the column vector of the fields in the both hand sides of the primitive unit cell as $\left(\Omega_{1}^{+}, \Omega_{1}^{-}\right)$and $\left(\Omega_{0}^{+}, \Omega_{0}^{-}\right)$, in the $T$-matrix algorithm we write

$$
\left(\begin{array}{l}
\Omega_{1}^{+} \\
\Omega_{1}^{-}
\end{array}\right)=T\left(\begin{array}{l}
\Omega_{0}^{+} \\
\Omega_{0}^{-}
\end{array}\right)=\left(\begin{array}{ll}
t_{11} & t_{12} \\
t_{21} & t_{22}
\end{array}\right)\left(\begin{array}{l}
\Omega_{0}^{+} \\
\Omega_{0}^{-}
\end{array}\right)
$$

From Bloch's theorem Eq. (2.35), we have $\Omega_{1}^{+}=e^{i k \cdot a_{3}} \Omega_{0}^{+}$, and $\Omega_{1}^{-}=e^{i k \cdot a_{3}} \Omega_{0}^{-}$. Under this condition, Eq. (2.36) becomes 


$$
T\left(\begin{array}{l}
\Omega_{0}^{+} \\
\Omega_{0}^{-}
\end{array}\right)=e^{i \mathbf{k} \cdot \mathbf{a}_{3}}\left(\begin{array}{c}
\Omega_{0}^{+} \\
\Omega_{0}^{-}
\end{array}\right)
$$

which means that the Bloch's phase factor is the eigenvalue of the $T$-matrix for a unit cell of the photonic crystal layer. However, as we have mentioned in the previous section, the $T$ matrix itself is not stable for thick grating, therefore, the solution of eigen-problem Eq. (2.36) is also not reliable. So we turn to the numerically stable $S$-matrix algorithm. From equation

$$
\left(\begin{array}{l}
\Omega_{1}^{+} \\
\Omega_{0}^{-}
\end{array}\right)=S\left(\begin{array}{l}
\Omega_{0}^{+} \\
\Omega_{1}^{-}
\end{array}\right)=\left(\begin{array}{ll}
S_{11} & S_{12} \\
S_{21} & S_{22}
\end{array}\right)\left(\begin{array}{l}
\Omega_{0}^{+} \\
\Omega_{1}^{-}
\end{array}\right)
$$

and using Bloch's theorem, we can derive

$$
\left(\begin{array}{cc}
S_{11} & 0 \\
S_{21} & -I
\end{array}\right)\left(\begin{array}{l}
\Omega_{0}^{+} \\
\Omega_{0}^{-}
\end{array}\right)=e^{i k \cdot \mathrm{a}_{3}}\left(\begin{array}{cc}
I & -S_{12} \\
0 & -S_{22}
\end{array}\right)\left(\begin{array}{l}
\Omega_{0}^{+} \\
\Omega_{0}^{-}
\end{array}\right)
$$

Eq. (2.39) is a generalized eigen-problem $A x=\lambda B x$, where $A$ and $B$ are both square matrices, $\lambda$ is the eigenvalue, and $x$ is the eigenvector. Eq. (2.39) can be written into another form:

$$
\left[\left(\begin{array}{cc}
S_{11} & 0 \\
S_{21} & -I
\end{array}\right)-e^{i \mathbf{k} \cdot \mathbf{a}_{3}}\left(\begin{array}{cc}
I & -S_{12} \\
0 & -S_{22}
\end{array}\right)\right]\left(\begin{array}{l}
\Omega_{0}^{+} \\
\Omega_{0}^{-}
\end{array}\right)=P\left(\begin{array}{l}
\Omega_{0}^{+} \\
\Omega_{0}^{-}
\end{array}\right)=0
$$

Then the eigen-problem is solved by setting $\operatorname{det}(P)=0$, or by finding the zero eigenvalue of the matrix $P$. 
In the second scheme Eq. (2.40), all components of the Bloch's wave vector $k_{x}, k_{y}$, and $k_{z}$ are given explicitly as input, the unknown variable is $\omega$. Therefore, the solution of the photonic band structure in this scheme can be classified as $\omega=f\left(k_{x}, k_{y}, k_{z}\right)$. This scheme is similar to the conventional Plane Wave Method in that given one Bloch's wave vector, the corresponding eigen-frequency are solved. However, there is a big difference. The eigenmatrix itself involves the unknown eigenvalue $\omega$. Therefore, the standard eigen-problem solution algorithm is not applicable, instead, one should use other root-searching algorithms of nonlinear equations to find the eigenvalues. There is a benefit as compensation for this numerical inconvenience: the current scheme can effectively deal with dispersive materials where $\varepsilon$ is dispersive with respect to the frequency. Obviously, using this scheme, we can account for the photonic band structure along any prefixed line in the first Brillouin zone (BZ) by only carrying out the transfer-matrix calculation along a single stacking direction. For example, we can generate the conventional 3D photonic band structure (namely, diagrams plotted along all high-symmetry lines in the first BZ) of a FCC lattice by only considering the (001) stacking direction of the crystal layers.

In the first scheme Eq. (2.39), the frequency $\omega$ and lateral components of the Block's wave vector $k_{x}$ and $k_{y}$ are explicitly given as an input, and the unknown variable is the Bloch wave vector component $k_{z}$. So the solution of the photonic band structure can be categorized as $k_{z}=f\left(\omega, k_{x}, k_{y}\right)$, where $k_{z}$ should be a real number, implying that the eigenvalue $e^{i \mathbf{k} \cdot \mathbf{a}_{3}}$ of Eq. (2.39) must be a complex number of unity modulus. In our numerical experiences, the calculated eigenvalue corresponding to a Bloch's mode is always of a 
modulus different from unity within $10^{-10}$, verifying the numerical stability of the planewave based $S$-matrix algorithm in application to the photonic band structure solution. This scheme can also apply to dispersive materials. Compared with the second scheme, this scheme is most numerically economic for the photonic band structure along a prefixed line in the first $\mathrm{BZ}$ parallel to the layer stacking direction. In this case, $k_{x}=c o n s t, k_{y}=c o n s t$, and $k_{z}=f(\omega)$. For example, for a FCC lattice, the $\Gamma-X$ band is solved by considering the (100) stacking direction of the crystal layers, while the $\Gamma-L$ band should be solved by using the (111) stacking direction. In the first scheme $k_{z}=f\left(k_{x}, k_{y}, \omega\right)$, the transfer-matrix is calculated exactly along the crystal stacking direction parallel to the high-symmetry lines in the standard diagram of band structures. In principle, several stacking directions are needed to account for the whole diagram. In the second scheme, $\omega=f\left(k_{x}, k_{y}, k_{z}\right)$, one can calculate the whole diagram of photonic band structures by looking at only one single stacking direction. In the former scheme, very reliable standard eigen-problem solution tools are ready for use, while in the latter scheme, consideration of only one stacking direction can reduce the numerical burden. More importantly, it is more flexible to select a stacking direction along which fast converged results can be achieved by using optimal Fourier expansion rules. It may be valuable if we can combine the advantages of these two schemes. Let us take the layer-by-layer photonic crystal as an example. To guarantee fast numerical convergence, we should consider the (001) stacking direction. Now for each Bloch's wave vector in the first $\mathrm{BZ}, \mathbf{k}=\left(k_{x}, k_{y}, k_{z}\right)$, which is usually lying at an arbitrary high-symmetry line, we project it onto the stacking direction, and obtain the lateral wave vector in this plane, which is $k_{x}$ and 
$k_{y}$. Then we use the eigen-problem solution scheme: $k_{z}^{\prime}=f\left(k_{x}, k_{y}, \omega\right)$ to calculate the dispersion along the (001) direction. Matching $k_{z}^{\prime}$ to the prefixed value of $k_{z}$ either through simple sorting or through interpolation technique, we can pick up the eigen-frequency corresponding to the wave vector $\mathbf{k}$, namely, we have arrived at the scheme $\omega=f\left(k_{x}, k_{y}, k_{z}\right)$. To speed up the numerical solution, each time we do not necessarily need to work in a very wide frequency range, such as $\left(0, \omega_{m}\right)$, where $\omega_{m}$ is the maximum frequency considered in the band structure calculations. Suppose we have obtained the eigenfrequency at one wave vector. Then we can start from this point, and carry out the above numerical procedure in the adjacent small frequency range to find out the eigen-frequency of the adjacent wave vector to the $Z$ point for each photonic band. Repeating this procedure, we can efficiently solve the whole diagram of photonic band structures.

\subsection{The Modal Expansion Method}

In this section we will introduce an analytic modal expansion method combined with a transfer-matrix technique to investigate the reflection, transmission, and absorption spectra of three-dimensional layer-by-layer metallic photonic crystals working in a infrared wavelengths regime. For a metallic photonic crystal structure, the usual plane-wave expansion method[10] becomes ineffective. Severe convergence difficulty is found for a highly-conducting $2 \mathrm{D}$ grating due to a skin depth two orders of magnitude smaller than the incident wavelength. To attack the theoretical challenge for a 2D metallic layer-by-layer grating scaled in mid-IR wavelengths, we introduce an EM approach which combines a 
series of techniques ranging from analytic modal expansion method, to the transfer-matrix method, and to the application of structural symmetry.

\subsubsection{In-Plane Incidence of Plane Waves}

To study the general scattering of EM waves by a 1D lamellar perfect-conducting grating under arbitrary incidence condition, it is helpful to look at the simplest case of the inplane incidence, where the incident wave vector lies in a plane perpendicular to the grating axis. The grating is extending along the $y$-axis direction, and repeats its unit cell along the $x$-axis direction every distance of $d$. The air-metal interface is located at $x=0$ and $x=a$, respectively. A plane wave is incident on the grating from up to down along the $-z$ direction with a wave vector $\mathbf{k}_{0}=\left(k_{0 x}, k_{0 z}\right)=k_{0}(\sin \theta,-\cos \theta)$, where $k_{0}=(\omega / c)$ is the wave number, $\omega$ is the angular frequency, $c$ is the light speed in vacuum, and $\theta$ is the incident angle, $0 \leq \theta<\pi / 2$. In this situation we have two eigenmodes for the scattering problem, the $\mathrm{TE}$ and TM modes, in which either the electric or the magnetic field is parallel to the grating axis. For the TE mode, the electric field in the incident region $(z>h)$, in the grating region ( $h \geq z \geq 0)$, and in the transmission region $(z<0)$, can be written as

$$
\begin{aligned}
& E_{r}(x, z)=\sum_{n=-\infty}^{\infty}\left[\delta_{n, 0} e^{-i \beta_{n}(z-h)}+r_{n} e^{i \beta_{n}(z-h)}\right] \psi_{n}(x), \\
& E_{m}(x, z)=\sum_{m=1}^{\infty}\left(A_{m} \sin \mu_{m} z+B_{m} \cos \mu_{m} z\right) X_{m}(x),
\end{aligned}
$$




$$
E_{t}(x, z)=\sum_{n=-\infty}^{\infty} t_{n} e^{i \beta_{n}(z-h)} \psi_{n}(x)
$$

Here $\psi_{n}(x)=d^{-1 / 2} e^{i\left(k_{0 x}+n 2 \pi d\right) x}$, and $X_{m}(x)=(2 / a)^{1 / 2} \sin \left(s_{m} x\right)$ with $s_{m}=m \pi / a, a$ being the width of the air domain. $\mu_{m}=\left(k_{0}^{2}-s_{m}^{2}\right)^{1 / 2}$ if $s_{m} \leq k_{0}$, and $\mu_{m}=i\left(s_{m}^{2}-k_{0}^{2}\right)^{1 / 2}$ if $s_{m}>k_{0}$. It can be directly checked that $E_{m}$ is the solution of the Helmholtz equation in each air domain and satisfies the boundary condition that at $x=0$ and $x=a, E_{m}=0$. To determine the unknown variables $r_{n}$ and $t_{n}$, the boundary conditions at the interfaces $z=h$ and $z=0$ are used, where the tangential electric field $E$ is continuous everywhere in the whole unit cell of the grating $0<x<d$, while the tangential magnetic field $H_{x}$ is only continuous in the air domain $0<x<a$. This means

$$
\begin{gathered}
E_{r}(x, h)=E_{m}(x, h), \quad 0<x<d, \\
\frac{\partial}{\partial z} E_{r}(x, h)=\frac{\partial}{\partial z} E_{m}(x, h), \quad 0<x<a, \\
E_{t}(x, 0)=E_{m}(x, 0), \quad 0<x<d, \\
\frac{\partial}{\partial z} E_{t}(x, 0)=\frac{\partial}{\partial z} E_{m}(x, 0), \quad 0<x<a,
\end{gathered}
$$

The so-called method of moment[17,18] is employed to solve these equations. In Eqs. (2.44a) and (2.44c) we project both hand sides onto the basis function $\psi_{n}(x)$ of the whole 
unit cell, while in Eqs. (2.44b) and (2.44d) projections onto the basis function $X_{m}(x)$ are used. This leads to equations

$$
\begin{gathered}
r_{q}+\delta_{q, 0}=\sum_{m=1}^{\infty}\left(A_{m} \sin \mu_{m} h+B_{m} \cos \mu_{m} h\right) J_{q m}^{*}, \\
\sum_{n=-\infty}^{\infty} i \beta_{n}\left(r_{n}-\delta_{n, 0}\right) J_{n p}=A_{p} \mu_{p} \cos \mu_{p} h-B_{p} \mu_{p} \sin \mu_{p} h, \\
t_{q}=\sum_{m=1}^{\infty} B_{m} J_{q m}^{*}, \\
\sum_{n=-\infty}^{\infty}-i \beta_{n} t_{n} J_{n p}=A_{p} \mu_{p} .
\end{gathered}
$$

Here $J_{n n}=\int_{0}^{a} \psi_{m}(x) X_{n}(x) d x$, and the superscript "**" denotes the complex conjugate. Deleting $A_{m}$ and $B_{m}$ from Eqs. (2.45a)-(2.45d) finally yields the following coupled linear equations

$$
\begin{gathered}
\sum_{n=-\infty}^{\infty}\left[\left(A_{q n}-\delta_{q, n}\right) r_{n}+B_{q n} t_{n}\right]=A_{q 0}+\delta_{q, 0}, \\
\sum_{n=-\infty}^{\infty}\left[B_{q n} r_{n}+\left(A_{q n}-\delta_{q, n}\right) t_{n}\right]=B_{q 0}
\end{gathered}
$$

where the matrix elements $A_{q n}$ and $B_{q n}$ are defined as 


$$
\begin{aligned}
& A_{q n}=-i \beta_{n} \sum_{m=1}^{\infty} \mu_{m}^{-1} \operatorname{ctan}\left(\mu_{m} h\right) J_{q m}^{*} J_{n m}, \\
& B_{q n}=-i \beta_{n} \sum_{m=1}^{\infty} \mu_{m}^{-1} \csc \left(\mu_{m} h\right) J_{q m}^{*} J_{n m} .
\end{aligned}
$$

The solution for the TM mode follows the similar procedure. The magnetic field $H_{y}$ is written into the plane-wave and modal expansions

$$
\begin{gathered}
H_{r}(x, z)=\sum_{n=-\infty}^{\infty}\left[\delta_{n, 0} e^{-i \beta_{n}(z-h)}+r_{n} e^{i \beta_{n}(z-h)}\right] \psi_{n}(x), \\
H_{m}(x, z)=\sum_{m=0}^{\infty}\left(A_{m} \sin \mu_{m} z+B_{m} \cos \mu_{m} z\right) Y_{m}(x), \\
H_{t}(x, z)=\sum_{n=-\infty}^{\infty} t_{n} e^{i \beta_{n}(z-h)} \psi_{n}(x),
\end{gathered}
$$

where $Y_{m}(x)=(2 / a)^{1 / 2} \cos \left(s_{m} x\right)$ for $m>0$, and $Y_{0}(x)=(1 / a)^{1 / 2}$. The expansion form of $H_{m}$ guarantees that the tangential electric field $E_{z}\left[\propto \frac{\partial}{\partial x} H_{m}\right]$ is zero at the wall of each air domain $x=0$ and $x=a$. The boundary conditions at interfaces $z=h$ and $z=0$ require

$$
\begin{gathered}
H_{r}(x, h)=H_{m}(x, h), \quad 0<x<a, \\
\frac{\partial}{\partial z} H_{r}(x, h)=\frac{\partial}{\partial z} H_{m}(x, h), \quad 0<x<d,
\end{gathered}
$$




$$
\begin{gathered}
H_{t}(x, 0)=H_{m}(x, 0), \quad 0<x<a, \\
\frac{\partial}{\partial z} H_{t}(x, 0)=\frac{\partial}{\partial z} H_{m}(x, 0), \quad 0<x<d .
\end{gathered}
$$

After some simple algebraic manipulations we obtain

$$
\begin{gathered}
\sum_{n=-\infty}^{\infty}\left[\left(A_{q n}-i \beta_{n} \delta_{q, n}\right) r_{n}+B_{q n} t_{n}\right]=i \beta_{q} \delta_{q, 0}-A_{q 0}, \\
\sum_{n=-\infty}^{\infty}\left[B_{q n} r_{n}+\left(A_{q n}-i \beta_{n} \delta_{q, n}\right) t_{n}\right]=-B_{q 0} .
\end{gathered}
$$

The matrix elements $A_{q n}$ and $B_{q n}$ are now defined as

$$
A_{q n}=\sum_{m=0}^{\infty} \mu_{m} \operatorname{ctan}\left(\mu_{m} h\right) J_{g m}^{*} J_{n m}
$$

and

$$
B_{q n}=-\sum_{m=0}^{\infty} \mu_{m} \csc \left(\mu_{m} h\right) J_{q m}^{*} J_{n m}
$$

where $J_{m n}$ is now defined as $J_{m n}=\int_{0}^{a} \psi_{m}(x) Y_{m}(x) d x$.

To solve numerically Eqs. (2.46) and (2.52), we have to truncate the infinite linear equations using a finite number of plane waves. It can be found from Eqs. (2.47a), (2.47b), (2.53a), and (2.53b) that $J_{n m}, \operatorname{ctan}\left(\mu_{m} h\right)$, and $\csc \left(\mu_{m} h\right)$ are all rapid decaying functions of $\mu_{m} h$. This allows one to use only moderate modal expansion number $M$ to obtain converged results of the matrix elements $A_{q n}$ and $B_{q n}$. In addition, looking into the symmetry of the 
coefficient matrix in Eqs. (2.46a) and (2.46b) for the TE mode, and Eqs. (2.52a) and (2.52b) for the TM mode, one can expect that stable and fast convergent solutions of the reflection and transmission spectra as well as EM fields can be achieved using truncation by only moderate orders of $r_{n}, t_{n}, A_{m}$ and $B_{m}$ for both the TE and TM modes.

After solution of $r_{n}$ and $t_{n}$, the modal coefficients $A_{m}$ and $B_{m}$ can be obtained directly by substituting $r_{n}$ and $t_{n}$ into Eqs. (2.45b) and (2.45d), etc. Finally, the transmission and reflection coefficients are calculated by

$$
T=\sum_{n} T_{n}=\sum_{n}\left|t_{n}\right|^{2}
$$

and

$$
R=\sum_{n} R_{n}=\sum_{n}\left|r_{n}\right|^{2}
$$

where the summation is run over those homogeneous Bragg waves with a lateral wave vector $\left|k_{0 x}+2 \pi n / d\right| \leq k_{0}$

\subsubsection{Off-Plane Conical Incidence of Plane Waves}

In the off-plane conical incidence configuration; the incident plane wave has a wave vector component along the grating axis. Suppose an EM wave with a wave vector $\mathbf{k}=\left(k_{0 x}, k_{0 y}, k_{0 z}\right)=k_{0}(\sin \theta \cos \phi, \sin \theta \sin \phi, \cos \theta)$ is incident on the perfect-conducting 1D grating, where $\theta$ and $\phi$ are the incident polar and azimuthal angles, with $\pi / 2 \leq \theta<\pi$, and 
$0 \leq \phi<2 \pi$. In the conical incidence, the TE and TM modes are no longer the eigenmodes of a 1D grating, instead, they are coupled with each other. Since the grating is homogeneous along the $y$-axis direction, the $y$-axis wave vector component is a constant $k_{0 y}$ in the process of the wave scattering.

The $\mathbf{E}$ and $\mathbf{H}$ fields in the incidence and transmission regions are now both composed of three components. We first consider EM fields in the incidence region $\mathbf{E}_{r}$ and $\mathbf{H}_{r}$. In the plane-wave basis, the tangential components of $\mathbf{E}_{r}$ are written in general forms as

$$
\begin{aligned}
& E_{x}(\mathbf{r})=\sum_{i=-\infty}^{\infty} E_{i j, x}(z) e^{i k_{k_{i x}} x+i k_{j y} y}=\sum_{i=-\infty}^{\infty}\left[E_{i j, x}^{+}(z)+E_{i j, x}^{-}(z)\right] e^{i k_{k} x+i k_{k y} y}, \\
& E_{y}(\mathbf{r})=\sum_{i=-\infty}^{\infty} E_{i j, y}(z) e^{i k_{k x} x+i k_{j y} y}=\sum_{i=-\infty}^{\infty}\left[E_{i j, y}^{+}(z)+E_{i j, y}^{-}(z)\right] e^{i k_{i x} x+i k_{j y} y} .
\end{aligned}
$$

Here $k_{i x}=k_{0 x}+i 2 \pi / d, k_{j y}=k_{0 y}, E_{i j, x(y)}^{+}(z)=E_{i j, x(y)}^{+} e^{i \beta_{y}(z-h)}, E_{i j, x(y)}^{-}(z)=E_{i j, x(y)}^{-} e^{-i \beta_{j j}(z-h)}$, where $\beta_{i j}=-\left(k_{0}^{2}-k_{i x}^{2}-k_{j y}^{2}\right)^{1 / 2} \quad$ for $\quad k_{0}^{2}-k_{i x}^{2}-k_{j y}^{2} \geq 0 \quad, \quad$ and $\quad \beta_{i j}=-i\left(k_{i x}^{2}+k_{j y}^{2}-k_{0}^{2}\right)^{1 / 2} \quad$ for $k_{0}^{2}-k_{i x}^{2}-k_{j y}^{2}<0$. The definition of $\beta_{i j}$ are in consistence with the fact that the incident wave is propagating along the $-z$ direction.

The $z$-component $E_{z}(\mathbf{r})$ can be obtained from $\nabla \cdot \mathbf{E}(\mathbf{r})=0$. The magnetic field can be derived from $\mathbf{H}=\left(1 / i k_{0}\right) \nabla \times \mathbf{E}$, and the tangential components are written as

$$
H_{x}(\mathbf{r})=\sum_{i=-\infty}^{\infty} H_{i j, x}(z) e^{i k_{x x} x+i k_{j y} y}=\sum_{i=-\infty}^{\infty}\left[H_{i j, x}^{+}(z)+H_{i j, x}^{-}(z)\right] e^{i k_{k x} x+i k_{j y} y},
$$




$$
H_{y}(\mathbf{r})=\sum_{i=-\infty}^{\infty} H_{i j, y}(z) e^{i k_{i x} x+i k_{j y} y}=\sum_{i=-\infty}^{\infty}\left[H_{i j, y}^{+}(z)+H_{i j, y}^{-}(z)\right] e^{i k_{i x} x+i k_{j y} y},
$$

where $H_{i j, x(y)}^{+}(z)=H_{i j, x(y)}^{+} e^{i \beta_{y j}(z-h)}, H_{i j, x(y)}^{-}(z)=H_{i j, x(y)}^{-} e^{-i \beta_{y}(z-h)}$. For each wave vector we have the following relation between the $\mathbf{H}$ and $\mathbf{E}$ fields: $\left(H_{i j, x}^{+}, H_{i j, y}^{+}\right)^{T}=T_{0, i j}\left(E_{i j, x}^{+}, E_{i j, y}^{+}\right)^{T}$, and $\left(H_{i j, x}^{-}, H_{i j, y}^{-}\right)^{T}=-T_{0, i j}\left(E_{i j, x}^{-}, E_{i j, y}^{-}\right)^{T}$, where the superscript ' $T^{\prime \prime}$ denotes matrix transposition. The $2 \times 2$ matrix $T_{0, i j}$ has matrix elements $T_{0, i j}^{11}=-k_{i x} k_{j y} /\left(k_{0} \beta_{i j}\right), T_{0, i j}^{12}=\left(k_{i x}^{2}-k_{0}^{2}\right) /\left(k_{0} \beta_{i j}\right)$, $T_{0, j}^{21}=\left(k_{0}^{2}-k_{j y}^{2}\right) /\left(k_{0} \beta_{i j}\right)$, and $T_{0, i j}^{22}=k_{i x} k_{j y} /\left(k_{0} \beta_{i j}\right)$. It can be seen that $E^{+}\left(H^{+}\right)$and $E^{-}\left(H^{-}\right)$ correspond to the incident and reflected waves, respectively.

The EM fields in the transmission region $\mathbf{E}_{t}$ and $\mathbf{H}_{t}$ have the same general form of expansion,

$$
\begin{aligned}
& E_{x}(\mathbf{r})=\sum_{i=-\infty}^{\infty} U_{i j, x}(z) e^{i k_{i x} x+i k_{j y} y}=\sum_{i=-\infty}^{\infty}\left[U_{i j, x}^{+}(z)+U_{i j, x}^{-}(z)\right] e^{i k_{i x} x+i k_{j y} y} \\
& E_{y}(\mathbf{r})=\sum_{i=-\infty}^{\infty} U_{i j, y}(z) e^{i k_{i x} x+i k_{j y} y}=\sum_{i=-\infty}^{\infty}\left[U_{i j, y}^{+}(z)+U_{i j, y}^{-}(z)\right] e^{i k_{i x} x+i k_{j y} y} \\
& H_{x}(\mathbf{r})=\sum_{i=-\infty}^{\infty} V_{i j, x}(z) e^{i k_{i j} x+i k_{j y} y}=\sum_{i=-\infty}^{\infty}\left[V_{i j, x}^{+}(z)+V_{i j, x}^{-}(z)\right] e^{i k_{j x} x+i k_{j y} y} \\
& H_{y}(\mathbf{r})=\sum_{i=-\infty}^{\infty} V_{i j, y}(z) e^{i k_{i x} x+i k_{j y} y}=\sum_{i=-\infty}^{\infty}\left[V_{i j, y}^{+}(z)+V_{i j, y}^{-}(z)\right] e^{i k_{i x} x+i k_{j y} y}
\end{aligned}
$$


where $U_{i j, x(y)}^{+}(z)=U_{i j, x(y)}^{+} e^{i \beta_{j, z}}, U_{i j, x(y)}^{-}(z)=U_{i j, x(y)}^{-} e^{-i \beta_{i j} z} \cdot V_{i j, x(y)}^{+}$and $V_{i j, x(y)}^{-}$are also connected to $U_{i j, x(y)}^{+}$and $U_{i j, x(y)}^{-}$through the $2 \times 2$ matrix $T_{0, i j}$. Obviously $U^{+}\left(V^{+}\right)$and $U^{-}\left(V^{-}\right)$ correspond to forward and backward propagating waves in the transmission region, respectively. Temporarily, we assume that both waves coexist in the transmission region. In reality, $U^{-}\left(V^{-}\right)$should vanish, since only transmitted waves exist in this region for our 1D lamellar grating. But we will see in later sections that the introduction of $U^{-}\left(V^{-}\right)$into a single-layer $1 \mathrm{D}$ grating will bring us great convenience and flexibility to the scattering problem of a general multilayer $2 \mathrm{D}$ grating, because they are one of the central elements in the transfer-matrix method.

As noted above, the $y$-component of the wave vector is a constant during the process of EM waves scattering by the 1D grating, therefore, the EM fields $\mathbf{E}_{m}$ and $\mathbf{H}_{m}$ inside the grating domain can be written as

$$
\begin{gathered}
E_{y}(\mathbf{r})=\sum_{m=1}^{\infty}\left(A_{m}^{j} \cos \mu_{m} z+B_{m}^{j} \sin \mu_{m} z\right) X_{m}(x) e^{i k_{j y} y}, \\
E_{x}(\mathbf{r})=\sum_{m=0}^{\infty}\left(E_{m}^{j} \cos \mu_{m} z+F_{m}^{j} \sin \mu_{m} z\right) Y_{m}(x) e^{i k_{j y} y}, \\
E_{z}(\mathbf{r})=\sum_{m=1}^{\infty}\left[\mu_{m}^{-1}\left(i k_{j y} B_{m}^{j}-F_{m}^{j} S_{m}\right) \cos \mu_{m} z+\mu_{m}^{-1}\left(-i k_{j y} A_{m}^{j}+E_{m}^{j} s_{m}\right) \sin \mu_{m} z\right] X_{m}(x) e^{i k_{j y} y},
\end{gathered}
$$

where, $\quad \mu_{m}$ is now defined as $\mu_{m}=\left(k_{0}^{2}-s_{m}^{2}-k_{j y}^{2}\right)^{1 / 2}$ if $s_{m}^{2}+k_{j y}^{2} \leq k_{0}^{2}$, and $\mu_{n}=i\left(s_{m}^{2}+k_{j y}^{2}-k_{0}^{2}\right)^{1 / 2}$ if $s_{m}^{2}+k_{j y}^{2}>k_{0}^{2}$. It can be shown that each expansion term in Eqs. 
(2.60)-(2.62) is a solution to Maxwell's equations in the rectangular air slit satisfying the boundary conditions that the tangential components $E_{y}=0$ and $E_{\bar{z}} 0$ at both $x=0$ and $x=a$. From Maxwell's equation $i k_{0} \mathbf{H}=\nabla \times \mathbf{E}$, we can derive the tangential $\mathbf{H}_{m}$ components as

$$
\begin{gathered}
i k_{0} H_{x}(\mathbf{r})=\frac{\partial}{\partial y} E_{z}-\frac{\partial}{\partial z} E_{y}=\sum_{m=1}^{\infty}\left(k_{j y}^{2} \mu_{m}^{-1}+\mu_{m}\right) \sin \mu_{m} z A_{m}^{j}-\left(k_{j y}^{2} \mu_{m}^{-1}+\mu_{m}\right) \cos \mu_{m} z B_{m}^{j} \\
+\left(i k_{j y} \mu_{m}^{-1} s_{m}\right) \sin \mu_{m} z E_{m}^{j}-\left(i k_{j y} \mu_{m}^{-1} s_{m}\right) \cos \mu_{m} z F_{m}^{j} \\
i k_{0} H_{y}(\mathbf{r})=\frac{\partial}{\partial z} E_{x}-\frac{\partial}{\partial z} E_{z}=\sum_{m=1}^{\infty}\left[\left(i k_{j y} \mu_{m}^{-1} s_{m}\right) \sin \mu_{m} z A_{m}^{j}-\left(i k_{j y} \mu_{m}^{-1} s_{m}\right) \cos \mu_{m} z B_{m}^{j}\right. \\
\left.-\left(s_{m}^{2} \mu_{m}^{-1}+\mu_{m}\right) \sin \mu_{m} z E_{m}^{j}+\left(s_{m}^{2} \mu_{m}^{-1}+\mu_{m}\right) \cos \mu_{m} z B_{m}^{j}\right] Y_{m}(x) e^{i k_{j y} y} \\
+\left(-\mu_{0} \sin \mu_{0} z E_{0}^{j}+\mu_{0} \cos \mu_{0} z F_{0}^{j}\right) Y_{0}(x) e^{i k_{j y} y} .
\end{gathered}
$$

We have finished the expansion of EM fields in the incidence, grating, and transmission regions. We can continue to find out the unknown field expansion coefficients in the three regions through match of boundary conditions at $z=h$ and $z=0$. Similar to the TE and TM modes in the case of in-plane incidence, we start from boundary conditions

$$
\mathbf{E}_{r}^{\text {tan }}(z=h)=\mathbf{E}_{m}^{\text {tan }}(z=h), \quad 0<x<d,
$$




$$
\begin{aligned}
& \mathbf{H}_{r}^{\text {tan }}(z=h)=\mathbf{H}_{m}^{\text {tan }}(z=h), \quad 0<x<a, \\
& \mathbf{E}_{t}^{\text {tan }}(z=0)=\mathbf{E}_{m}^{\text {tan }}(z=0), \quad 0<x<d, \\
& \mathbf{H}_{r}^{\text {ran }}(z=0)=\mathbf{H}_{m}^{\text {ran }}(z=0), \quad 0<x<a,
\end{aligned}
$$

where the superscript " $\tan$ " means the tangential components of fields. Using the technique of moments at $z=h$ and $z=0$ for the electric field, we arrive at the following matrix equation after truncation over the infinite linear equations,

$$
d Z_{0}^{j}=P_{1} Z_{m}^{j}
$$

$Z_{0}^{j}$ and $Z_{m}^{j}$ are column vectors composed of the field expansion coefficients, where the superscript " $j$ " refers to the $k_{j y}$ component of the incident wave vector. They are defined by

$$
\begin{gathered}
Z_{0}^{j}=\left[E_{i j, x}(h), E_{i j, y}(h), U_{i j, x}(0), U_{i j, y}(0)\right]^{T}, \\
Z_{m}^{j}=\left(A_{m}^{j}, B_{m}^{j}, E_{0}^{j}, E_{m}^{j}, F_{0}^{j}, F_{m}^{j}\right)^{T},
\end{gathered}
$$

where $E_{i j, x(y)}(h)=\left[E_{-N j, x(y)}(h), \cdots, E_{0 j, x(y)}(h), \cdots, E_{N j, x(y)}(h)\right], A_{m}^{j}=\left(A_{1}^{j}, A_{2}^{j}, \cdots, A_{M-1}^{j}\right)$, etc. The corresponding dimension is $4 N_{0}$ and $4 M-2$, where $N_{0}=2 N+1$ and $M$ is the plane wave 
and modal numbers in the incidence and grating regions. The $\left(4 N_{0}\right) \times(4 M-2)$ dimensioned matrix $P_{1}$ is

$$
P_{1}=\left(\begin{array}{cccccc}
0 & 0 & \cos \left(\mu_{0} h\right) J_{i 0}^{*} & \cos \left(\mu_{m} h\right) J_{i m} & \sin \left(\mu_{0} h\right) J_{i 0}^{*} & \sin \left(\mu_{m} h\right) J_{i m} \\
\cos \left(\mu_{m} h\right) I_{i m}^{*} & \sin \left(\mu_{m} h\right) I_{i m}^{*} & 0 & 0 & 0 & 0 \\
0 & 0 & J_{i 0}^{*} & J_{i m}^{*} & 0 & 0 \\
I_{i m}^{*} & 0 & 0 & 0 & 0 & 0
\end{array}\right)
$$

where each element (such as $J_{i m}^{*}$ ) represents a matrix, and the multiplication such as $J_{i m}^{*} E_{m}^{j}$ implies summation over the index $m$, which is just the multiplication of a matrix and a column vector. Here $I_{i m}$ and $J_{i m}$ are defined as $I_{i m}=\int_{0}^{a} e^{i k_{k x} x} X_{m}(x) d x$ and $J_{i m}=\int_{0}^{a} e^{i k_{k} x} Y_{m}(x) d x$. Matching the boundary conditions for the magnetic field at the interfaces $z=h$ and $z=0$, we obtain

$$
i k_{0} P_{2} Z_{1}^{j}=P_{3} Z_{m}^{j}
$$

where $Z_{1}^{j}=\left[H_{i j, x}(h), H_{i j, y}(h), V_{i j, x}(0), V_{i j, y}(0)\right]^{T}$.

$P_{2}$ is a $(4 M-2) \times\left(4 N_{0}\right)$ matrix defined by 


$$
P_{2}=\left(\begin{array}{cccc}
I_{i m} & 0 & 0 & 0 \\
0 & J_{i m} & 0 & 0 \\
0 & J_{i 0} & 0 & 0 \\
0 & 0 & I_{i m} & 0 \\
0 & 0 & 0 & J_{i 0} \\
0 & 0 & 0 & J_{i m}
\end{array}\right)
$$

where $I_{i m}$ denotes a $(M-1) \times N_{0}$ matrix $G$ whose matrix element is $G_{m i}=I_{i m}$, and the others have similar implications.

$P_{3}$ is a $(4 M-2) \times(4 M-2)$ matrix defined by

$$
P_{3}=\left(\begin{array}{cccccc}
Q_{11} & Q_{12} & 0 & Q_{14} & 0 & Q_{16} \\
Q_{21} & Q_{22} & 0 & Q_{24} & 0 & Q_{26} \\
0 & 0 & Q_{33} & 0 & Q_{35} & 0 \\
0 & Q_{42} & 0 & 0 & 0 & Q_{46} \\
0 & 0 & 0 & 0 & Q_{55} & 0 \\
0 & Q_{62} & 0 & 0 & 0 & Q_{66}
\end{array}\right)
$$

where each block matrix $Q_{i j}$ is diagonal. The diagonal elements are

$$
\begin{aligned}
& Q_{11, n m}=\left(k_{j y}^{2} \mu_{m}^{-1}+\mu_{m}\right) \sin \left(\mu_{m} h\right), Q_{12, n m}=-\left(k_{j y}^{2} \mu_{m}^{-1}+\mu_{m}\right) \cos \left(\mu_{m} h\right), Q_{14, m m}=i k_{j y} \mu_{m}^{-1} s_{m} \sin \left(\mu_{m} h\right), \\
& Q_{16, m m}=-i k_{j y} \mu_{m}^{-1} s_{m} \cos \left(\mu_{m} h\right), Q_{21, m m}=i k_{j y} \mu_{m}^{-1} s_{m} \sin \left(\mu_{m} h\right), Q_{22, m m}=-i k_{j y} \mu_{m}^{-1} s_{m} \cos \left(\mu_{m} h\right), \\
& Q_{24, m m m}=-\left(s_{m}^{2} m u_{m}^{-1}+\mu_{m}\right) \sin \left(\mu_{m} h\right), Q_{26, m m}=\left(s_{m}^{2} m u_{m}^{-1}+\mu_{m}\right) \cos \left(\mu_{m} h\right), Q_{33, m m}=-\mu_{0} \sin \left(\mu_{0} h\right), \\
& Q_{35, n m m}=\mu_{0} \cos \left(\mu_{0} h\right), Q_{42, m m}=-\left(k_{j y}^{2} \mu_{m}^{-1}+\mu_{m}\right), Q_{46, m m}=-i k_{j y} \mu_{m}^{-1} s_{m}, Q_{55, m m}=\mu_{0}, \\
& Q_{62, n m m}=-i k_{j y} \mu_{m}^{-1} s_{m}, \text { and } Q_{66, m m}=s_{m}^{2} \mu_{m}^{-1}+\mu_{m} .
\end{aligned}
$$


To solve the transmission and reflection spectra, one can first delete the modal variables from Eqs. (2.66) and (2.67). This can be done by first calculating the inverse of $P_{3}$ by means of analytical manipulation, then substituting $Z_{m}^{j}$ back into Eq. (2.66). We finally obtain the following linear equations satisfied by the plane-wave expansion coefficients

$$
Z_{0}^{j}=P^{j} Z_{1}^{j}
$$

where the $\left(4 N_{0}\right) \times\left(4 N_{0}\right)$ coefficient matrix reads

$$
P^{j}=\left(\begin{array}{llll}
P_{11} & P_{12} & P_{13} & P_{14} \\
P_{21} & P_{22} & P_{23} & P_{24} \\
P_{31} & P_{32} & P_{33} & P_{34} \\
P_{41} & P_{42} & P_{43} & P_{44}
\end{array}\right) .
$$

The block matrices $P_{i j}(i, j=1,4)$ each is of dimension $N_{0} \times N_{0}$. They are defined as

$$
\begin{gathered}
P_{11}^{i i^{\prime}}=-\left(i k_{0} / d\right) \sum_{m=1}^{M-1} \operatorname{ctan}\left(\mu_{m} h\right) Z_{m}^{(21)} J_{i m}^{*} I_{i^{\prime} m}, \\
P_{12}^{i^{\prime}}=-\left(i k_{0} / d\right) \mu_{0}^{-1} \operatorname{ctan}\left(\mu_{0} h\right) J_{i 0}^{*} J_{i^{\prime} 0}-\left(i k_{0} / d\right) \sum_{m=1}^{M-1} \operatorname{ctan}\left(\mu_{m} h\right) Z_{m}^{(22)} J_{i m}^{*} J_{i^{\prime} m},
\end{gathered}
$$




$$
\begin{gathered}
P_{13}^{i i^{\prime}}=\left(i k_{0} / d\right) \sum_{m=1}^{M-1} \csc \left(\mu_{m} h\right) Z_{m}^{(21)} J_{i m}^{*} I_{i^{\prime} m}, \\
P_{14}^{i i^{\prime}}=\left(i k_{0} / d\right) \mu_{0}^{-1} \csc \left(\mu_{0} h\right) J_{i 0}^{*} J_{i^{\prime} 0}-\left(i k_{0} / d\right) \sum_{m=1}^{M-1} \csc \left(\mu_{m} h\right) Z_{m}^{(22)} J_{i m}^{*} J_{i^{\prime} m}, \\
P_{21}^{i i^{\prime}}=-\left(i k_{0} / d\right) \sum_{m=1}^{M-1} \operatorname{ctan}\left(\mu_{m} h\right) Z_{m}^{(11)} I_{i m}^{*} I_{i^{\prime} m}, \\
P_{22}^{i i^{\prime}}=-\left(i k_{0} / d\right) \sum_{m=1}^{M-1} \operatorname{ctan}\left(\mu_{m} h\right) Z_{m}^{(12)} I_{i m}^{*} J_{i^{\prime} m}, \\
P_{23}^{i i^{\prime}}=\left(i k_{0} / d\right) \sum_{m=1}^{M-1} \csc \left(\mu_{m} h\right) Z_{m}^{(11)} I_{i m}^{*} I_{i^{\prime} m}, \\
P_{24}^{i i^{\prime}}=\left(i k_{0} / d\right) \sum_{m=1}^{M-1} \csc \left(\mu_{m} h\right) Z_{m}^{(12)} I_{i m}^{*} J_{i^{\prime} m},
\end{gathered}
$$

where

$$
\begin{gathered}
Z_{m}^{(11)}=\left(s_{m}^{2} \mu_{m}^{-1}+\mu_{m}\right) / k_{0}^{2}, \quad Z_{m}^{(12)}=i k_{j y} \mu_{m}^{-1} s_{m} / k_{0}^{2}, \\
Z_{m}^{(21)}=-i k_{j y} \mu_{m}^{-1} s_{m} / k_{0}^{2}, \quad Z_{m}^{(22)}=-\left(k_{j y}^{2} \mu_{m}^{-1}+\mu_{m}\right) / k_{0}^{2} .
\end{gathered}
$$

Other matrices are given according to the following symmetry relations: 


$$
\begin{aligned}
& P_{31}^{i i^{\prime}}=-P_{13}^{i i^{\prime}}, \quad P_{32}^{i i^{\prime}}=-P_{14}^{i i^{\prime}}, \\
& P_{33}^{i i^{\prime}}=-P_{11}^{i i^{\prime}}, \quad P_{34}^{i i^{\prime}}=-P_{12}^{i i^{\prime}}, \\
& P_{41}^{i i^{\prime}}=-P_{23}^{i i^{\prime}}, \quad P_{42}^{i i^{\prime}}=-P_{24}^{i i^{\prime}}, \\
& P_{43}^{i i^{\prime}}=-P_{21}^{i i^{\prime}}, \quad P_{44}^{i i^{\prime}}=-P_{22}^{i i^{\prime}} .
\end{aligned}
$$

Now inserting into Eq. (2.28) the definition of $Z_{0}^{j}$ and $Z_{1}^{j}$ with respect to $E_{i j, x}(h), E_{i j, y}(h)$, $U_{i j, x}(0), U_{i j, y}(0), H_{i j, x}(h), H_{i j, y}(h), V_{i j, x}(0)$, and $V_{i j, y}(0)$, and using the relation between $E_{i j}$ and $H_{i j}, U_{i j}$ and $V_{i j}$, we get

$$
\left(\begin{array}{c}
\psi_{j}^{+} \\
\chi_{j}^{+}
\end{array}\right)=\left(\begin{array}{ll}
P_{11}^{j} T_{0}^{j} & P_{12}^{j} T_{0}^{j} \\
P_{21}^{j} T_{0}^{j} & P_{22}^{j} T_{0}^{j}
\end{array}\right)\left(\begin{array}{c}
\psi_{j}^{-} \\
\chi_{j}^{-}
\end{array}\right),
$$

where $\psi_{j}^{+}, \chi_{j}^{+}, \psi_{j}^{-}$, and $\chi_{j}^{-}$are $2 N_{0}$-dimensioned column vectors defined by

$$
\begin{aligned}
& \psi_{j}^{+}=\left(\cdots, E_{0 j, x}^{+}, E_{0 j, y}^{+}, \cdots, E_{i j, x}^{+}, E_{i j, y}^{+}, \cdots\right)^{T}+\left(\cdots, E_{0 j, x}^{-}, E_{0 j, y}^{-}, \cdots, E_{i j, x}^{-}, E_{i j, y}^{-}, \cdots\right)^{T}, \\
& \psi_{j}^{-}=\left(\cdots, E_{0 j, x}^{+}, E_{0 j, y}^{+}, \cdots, E_{i j, x}^{+}, E_{i j, y}^{+}, \cdots\right)^{T}-\left(\cdots, E_{0 j, x}^{-}, E_{0 j, y}^{-}, \cdots, E_{i j, x}^{-}, E_{i j, y}^{-}, \cdots\right)^{T},
\end{aligned}
$$




$$
\begin{aligned}
& \chi_{j}^{+}=\left(\cdots, U_{0 j, x}^{+}, U_{0 j, y}^{+}, \cdots, U_{i j, x}^{+}, U_{i j, y}^{+}, \cdots\right)^{T}+\left(\cdots, U_{0 j, x}^{-}, U_{0 j, y}^{-}, \cdots, U_{i j, x}^{-}, U_{i j, y}^{-}, \cdots\right)^{T}, \\
& \chi_{j}^{-}=\left(\cdots, U_{0 j, x}^{+}, U_{01 j, y}^{+}, \cdots, U_{i j, x}^{+}, U_{i j, y}^{+} \cdots\right)^{T}-\left(\cdots, U_{0 j, x}^{-}, U_{0 j, y}^{-}, \cdots, U_{i j, x}^{-}, U_{i j, y}^{-}, \cdots\right)^{T} .
\end{aligned}
$$

The index $i$ ranges inside $-N \leq i \leq N . T_{0}^{j}$ is a $\left(2 N_{0} \times 2 N_{0}\right)$-dimensioned block-diagonal matrix consisting of $\left\{T_{0, i j}, i=-N, N\right\}$ at its diagonal positions,

$$
T_{0}^{j}=\left(\begin{array}{ccccc}
T_{0,-N j} & \cdots & 0 & \cdots & 0 \\
\cdots & \cdots & \cdots & \cdots & \cdots \\
\cdots & 0 & T_{0,0 j} & 0 & \cdots \\
\cdots & \cdots & \cdots & \cdots & \cdots \\
0 & \cdots & 0 & \cdots & T_{0, N j}
\end{array}\right)
$$

For a one-layer 1D grating slab, we recognize that $\psi_{j}^{+}=E_{0}^{j}+E_{r}^{j}, \psi_{j}^{-}=E_{0}^{j}-E_{r}^{j}$, $\chi_{j}^{+}=\chi_{j}^{-}=E_{t}^{j}$, where $E_{0}^{j}, E_{r}^{j}$, and $E_{t}^{j}$ are column vectors consisting of the coefficients for the incident, reflected, and transmitted waves. Since in the usual diffraction problem only zero-order wave is incident on the grating, we can set $E_{0}^{j}=\left(0,0, \cdots, 0,0, E_{0 x}, E_{0 y}, 0,0, \cdots, 0,0\right)$ where $E_{0 x}$ and $E_{0 y}$ are the amplitudes of the incident electric field components. From Eq. (2.69) we find that $E_{r}^{j}$ and $E_{t}^{j}$ satisfy 


$$
\begin{gathered}
\left(\begin{array}{cc}
I+P_{11}^{j} T_{0}^{j} & -P_{12}^{j} T_{0}^{j} \\
P_{21}^{j} T_{0}^{j} & I-P_{22}^{j} T_{0}^{j}
\end{array}\right)\left(\begin{array}{c}
E_{r}^{j} \\
E_{t}^{j}
\end{array}\right)=\left(\begin{array}{cc}
P_{11}^{j} T_{0}^{j}-I & P_{12}^{j} T_{0}^{j} \\
P_{21}^{j} T_{0}^{j} & P_{22}^{j} T_{0}^{j}-I
\end{array}\right)\left(\begin{array}{c}
E_{0}^{j} \\
0
\end{array}\right) \\
=\left(\begin{array}{c}
\left(P_{11}^{j} T_{0}^{j}-I\right) E_{0}^{j} \\
P_{21}^{j} T_{0}^{j} E_{0}^{j}
\end{array}\right),
\end{gathered}
$$

where $I$ is a unit matrix. The simultaneous linear equations Eq. (2.30) can be solved via the standard Gaussian elimination method. Numerical experiences indicate that the numerical convergence for the off-plane scattering problem is as fast as for the in-plane incidence situation. This further verifies the powerful strength of this analytical modal method.

\subsubsection{Metallic Layer-by-Layer Photonic Crystal Gratings in Mid-Infrared Regimes}

When a metallic grating is working in the mid-IR regime, the metal is finiteconducting but has a very small skin depth. The key point is to solve the eigenmodes inside each 1D lamellar grating under arbitrary incident conditions. To achieve this, we use the following trial function as an eigenmode in a metallic grating consisting of infinitely-long rectangular rods along the $y$-axis direction,

$$
\begin{aligned}
& E_{y}(\mathbf{r})=e^{i k_{z} z+i k_{y} y}\left[A_{1} \sin \left(\beta_{1} x\right)+B_{1} \cos \left(\beta_{1} x\right)\right], \\
& E_{z}(\mathbf{r})=e^{i k_{z} z+i k_{y} y}\left[C_{1} \sin \left(\beta_{1} x\right)+D_{1} \cos \left(\beta_{1} x\right)\right]
\end{aligned}
$$




$$
\begin{gathered}
E_{x}(\mathbf{r})=e^{i k_{z} z+i k_{y} y}\left[-\left(i k_{y} B_{1}+i k_{z} D_{1}\right) \beta_{1}^{-1} \sin \left(\beta_{1} x\right)+\left(i k_{y} A_{1}+i k_{z} B_{1}\right) \beta_{1}^{-1} \cos \left(\beta_{1} x\right)\right], \\
i k_{0} H_{y}(\mathbf{r})=e^{i k_{z} z+i k_{y} y}\left[k_{z}\left(k_{y} B_{1}+k_{z} D_{1}\right) \beta_{1}^{-1} \sin \left(\beta_{1} x\right)-k_{z}\left(k_{y} A_{1}+k_{z} C_{1}\right) \beta_{1}^{-1} \cos \left(\beta_{1} x\right)\right. \\
\left.-\beta_{1} C_{1} \cos \left(\beta_{1} x\right)+\beta_{1} D_{1} \sin \left(\beta_{1} x\right)\right], \\
i k_{0} H_{z}(\mathbf{r})=e^{i k_{z} z+i k_{y} y}\left[-k_{y}\left(k_{y} B_{1}+k_{z} D_{1}\right) \beta_{1}^{-1} \sin \left(\beta_{1} x\right)+k_{y}\left(k_{y} A_{1}+k_{z} C_{1}\right) \beta_{1}^{-1} \cos \left(\beta_{1} x\right)\right. \\
\left.+\beta_{1} A_{1} \cos \left(\beta_{1} x\right)-\beta_{1} B_{1} \sin \left(\beta_{1} x\right)\right], \\
i k_{0} H_{x}(\mathbf{r})=e^{i k_{z} z+i k_{y} y}\left[\left(i k_{y} C_{1}-i k_{z} A_{1}\right) \sin \left(\beta_{1} x\right)+\left(i k_{y} D_{1}-i k_{z} B_{1}\right) \cos \left(\beta_{1} x\right)\right],
\end{gathered}
$$

for the E-field and H-field in the air domain $0 \leq x<a$. Here $k_{z}^{2}+\beta_{1}^{2}+k_{y}^{2}=\varepsilon_{1} k_{0}^{2}, \operatorname{Im}\left(\beta_{1}\right)>0$, and $\varepsilon_{1}=1$ is the dielectric constant of the air domain. The EM fields in the metal domain $a \leq x<d$ are

$$
\begin{aligned}
& E_{y}(\mathbf{r})=e^{i k_{z} z+i k_{y} y}\left[A_{2} e^{i \beta_{2}(x-a)}+B_{2} e^{-i \beta_{2}(x-d)}\right], \\
& E_{z}(\mathbf{r})=e^{i k_{z} z+i k_{y} y}\left[C_{2} e^{i \beta_{2}(x-a)}+D_{2} e^{-i \beta_{2}(x-d)}\right]
\end{aligned}
$$




$$
\begin{gathered}
E_{x}(\mathbf{r})=e^{i k_{z} z+i k_{y} y}\left[-\left(k_{y} A_{2}+k_{z} C_{2}\right) \beta_{2}^{-1} e^{i \beta_{2}(x-a)}+\left(k_{y} B_{2}+k_{z} D_{2}\right) \beta_{2}^{-1} e^{-i \beta_{2}(x-d)}\right] \\
i k_{0} H_{y}(\mathbf{r})=e^{i k_{z} z+i k_{y} y}\left[-i k_{y} k_{z} \beta_{2}^{-1} A_{2} e^{i \beta_{2}(x-a)}+i k_{y} k_{z} \beta_{2}^{-1} B_{2} e^{-i \beta_{2}(x-d)}\right. \\
\left.-\left(i k_{z}^{2} \beta_{2}^{-1}+i \beta_{2}\right) C_{2} e^{i \beta_{2}(x-a)}+\left(i k_{z}^{2} \beta_{2}^{-1}+i \beta_{2}\right) D_{2} e^{-i \beta_{2}(x-d)}\right] \\
i k_{0} H_{z}(\mathbf{r})=e^{i k_{z} z+i k_{y} y}\left[i k_{y} k_{z} \beta_{2}^{-1} C_{2} e^{i \beta_{2}(x-a)}-i k_{y} k_{z} \beta_{2}^{-1} D_{2} e^{-i \beta_{2}(x-d)}\right. \\
\left.+\left(i k_{y}^{2} \beta_{2}^{-1}+i \beta_{2}\right) A_{2} e^{i \beta_{2}(x-a)}-\left(i k_{y}^{2} \beta_{2}^{-1}+i \beta_{2}\right) B_{2} e^{-i \beta_{2}(x-d)}\right] \\
i k_{0} H_{x}(\mathbf{r})=e^{i i k_{z} z+i k_{y} y}\left[\left(i k_{y} C_{2}-i k_{z} A_{2}\right) e^{i \beta_{2}(x-a)}+\left(i k_{y} D_{2}-i k_{z} B_{2}\right) e^{-i \beta_{2}(x-d)}\right]
\end{gathered}
$$

Here $k_{z}^{2}+\beta_{2}^{2}+k_{y}^{2}=\varepsilon_{2} k_{0}^{2}, \operatorname{Im}\left(\beta_{2}\right)>0$, and $\varepsilon_{2}$ is the dielectric constant of the metal domain, which has strong dispersion. In writing down the above trial solution, we have noticed that $k_{z}$ is a tangential wave vector component along the metal wall, and thus is invariant across this air-metal interface.

To determine the amplitudes in the trial solution, boundary conditions at metal walls located at $x=a$ and $x=d$ are used. $E_{y}, E_{z}, H_{y}$ and $H_{z}$ are continuous across the metal walls. So we have

$$
A_{1} \sin \left(\beta_{1} a\right)+B_{1} \cos \left(\beta_{1} a\right)=A_{2}
$$




$$
\begin{gathered}
C_{1} \sin \left(\beta_{1} a\right)+D_{1} \cos \left(\beta_{1} a\right)=C_{2}, \\
p_{0} B_{1}=B_{2}, \\
p_{0} D_{1}=D_{2},
\end{gathered}
$$

for the E-field continuity, where $p_{0}=e^{i k_{0 x} d}$ is the Bloch's phase factor. In deriving Eqs. (4.84) and (4.85), we have used Bloch's theorem to relate fields at $x=0$ and $x=d$. In addition, we have neglected terms with a factor $e^{i \beta_{2}(d-a)}$, which is a small number due to the far larger metal domain width compared to the skin depth of metal in the mid-infrared wavelength regime. The continuity of the $\mathrm{H}$-field leads to

$$
\begin{gathered}
-k_{y} k_{z} \beta_{1}^{-1} \cos \left(\beta_{1} a\right) A_{1}+k_{y} k_{z} \beta_{1}^{-1} \sin \left(\beta_{1} a\right) B_{1}-\left(k_{z}^{2} \beta_{1}^{-1}+\beta_{1}\right) \cos \left(\beta_{1} a\right) C_{1} \\
+\left(k_{z}^{2} \beta_{1}^{-1}+\beta_{1}\right) \sin \left(\beta_{1} a\right) D_{1}=-i k_{y} k_{z} \beta_{2}^{-1} A_{2}-\left(k_{z}^{2} \beta_{2}^{-1}+\beta_{2}\right) C_{2} \\
k_{y} k_{z} \beta_{1}^{-1} \cos \left(\beta_{1} a\right) C_{1}-k_{y} k_{z} \beta_{1}^{-1} \sin \left(\beta_{1} a\right) D_{1}+\left(k_{y}^{2} \beta_{1}^{-1}+\beta_{1}\right) \cos \left(\beta_{1} a\right) A_{1} \\
-\left(k_{y}^{2} \beta_{1}^{-1}+\beta_{1}\right) \sin \left(\beta_{1} a\right) B_{1}=i k_{y} k_{z} \beta_{2}^{-1} B_{2}+\left(k_{y}^{2} \beta_{2}^{-1}+\beta_{2}\right) D_{2} \\
p_{0}\left[-k_{y} k_{z} \beta_{1}^{-1} A_{1}-\left(k_{z}^{2} \beta_{1}^{-1}+\beta_{1}\right) C_{1}\right]=i k_{y} k_{z} \beta_{2}^{-1} B_{2}+\left(k_{z}^{2} \beta_{2}^{-1}+\beta_{2}\right) D_{2}
\end{gathered}
$$




$$
p_{0}\left[k_{y} k_{z} \beta_{1}^{-1} C_{1}-\left(k_{y}^{2} \beta_{1}^{-1}+\beta_{1}\right) A_{1}\right]=-i k_{y} k_{z} \beta_{2}^{-1} D_{2}-\left(k_{y}^{2} \beta_{2}^{-1}+\beta_{2}\right) B_{2}
$$

Defining $2 \times 2$ matrices

$$
T_{1}=\left[\begin{array}{cc}
-k_{y} k_{z} \beta_{1}^{-1} & -\left(k_{z}^{2} \beta_{1}^{-1}+\beta_{1}\right) \\
k_{y}^{2} \beta_{1}^{-1}+\beta_{1} & k_{y} k_{z} \beta_{1}^{-1}
\end{array}\right],
$$

and

$$
T_{2}=\left[\begin{array}{cc}
-i k_{y} k_{z} \beta_{2}^{-1} & -i\left(k_{z}^{2} \beta_{2}^{-1}+\beta_{2}\right) \\
i\left(k_{y}^{2} \beta_{2}^{-1}+\beta_{2}\right) & i k_{y} k_{z} \beta_{2}^{-1}
\end{array}\right],
$$

we can derive from Eqs. (2.82)-(2.89) the following equations

$$
\begin{gathered}
\left(\begin{array}{l}
A_{2} \\
C_{2}
\end{array}\right)=T_{2}^{-1} T_{1}\left(\begin{array}{l}
A_{1} \\
C_{1}
\end{array}\right) \cos \left(\beta_{1} a\right)-T_{2}^{-1} T_{1}\left(\begin{array}{l}
B_{1} \\
D_{1}
\end{array}\right) \sin \left(\beta_{1} a\right), \\
\left(\begin{array}{l}
A_{2} \\
C_{2}
\end{array}\right)=\left(\begin{array}{l}
A_{1} \\
C_{1}
\end{array}\right) \sin \left(\beta_{1} a\right)+\left(\begin{array}{l}
B_{1} \\
D_{1}
\end{array}\right) \cos \left(\beta_{1} a\right),
\end{gathered}
$$

and

$$
\left(\begin{array}{l}
B_{1} \\
D_{1}
\end{array}\right)=-T_{2}^{-1} T_{1}\left(\begin{array}{l}
A_{1} \\
C_{1}
\end{array}\right)
$$

Designating $T=T_{2}^{-1} T_{1}$, and deleting $B_{1}$ and $D_{1}$ from Eqs. (2.92)-(2.94), we finally have 


$$
\left(I-T^{2}\right)\left(\begin{array}{l}
A_{1} \\
C_{1}
\end{array}\right) \sin \left(\beta_{1} a\right)=2 T\left(\begin{array}{l}
A_{1} \\
C_{1}
\end{array}\right) \cos \left(\beta_{1} a\right)
$$

or

$$
\operatorname{tg}\left(\beta_{1} a\right)\left(\begin{array}{l}
A_{1} \\
C_{1}
\end{array}\right)=2\left(I-T^{2}\right)^{-1} T\left(\begin{array}{l}
A_{1} \\
C_{1}
\end{array}\right)
$$

Eq. (2.96) is recognized to be a standard eigen-equation for the matrix $Q=2\left(I-T^{2}\right)^{-1} T$, with $\operatorname{tg}\left(\beta_{1} a\right)$ being the eigenvalue. It will be shown that this eigen-equation can be analytically solved. Notice $Q$ and $T$ has the same eigen-vector, it suffices to work on $T$, whose explicit form is

$$
T=T_{2}^{-1} T_{1}=\frac{-i}{\varepsilon_{2} k_{0}^{2}}\left[\begin{array}{cc}
k_{z}^{2} \beta_{2}^{-1} \beta_{1}+k_{y}^{2} \beta_{2} \beta_{1}^{-1}+\beta_{1} \beta_{2} & k_{y} k_{z}\left(\beta_{1}^{-1} \beta_{2}-\beta_{2}^{-1} \beta_{1}\right) \\
k_{y} k_{z}\left(\beta_{1}^{-1} \beta_{2}-\beta_{2}^{-1} \beta_{1}\right) & k_{y}^{2} \beta_{2}^{-1} \beta_{1}+k_{z}^{2} \beta_{2} \beta_{1}^{-1}+\beta_{1} \beta_{2}
\end{array}\right] .
$$

After some algebraic manipulations, we find that the eigen-equation

$$
T\left(\begin{array}{l}
A_{1} \\
C_{1}
\end{array}\right)=x\left(\begin{array}{l}
A_{1} \\
C_{1}
\end{array}\right)
$$

has eigenvalues of $x=-i \varepsilon_{1} \beta_{2} / \varepsilon_{2} \beta_{1}$ and $x=-i \beta_{1} / \beta_{2}$, corresponding to eigen-vectors $\left(A_{1}, C_{1}\right)=\left(1,-k_{y} / k_{z}\right)$ and $\left(A_{1}, C_{1}\right)=\left(k_{y} / k_{z}, 1\right)$, respectively. From this, the eigenvalues of Eq. (4.25) are directly calculated as 


$$
\operatorname{tg}\left(\beta_{1} a\right)=\frac{-2 i \beta_{1} \beta_{2}}{\beta_{1}^{2}+\beta_{2}^{2}}
$$

and

$$
\operatorname{tg}\left(\beta_{1} a\right)=\frac{-2 i \varepsilon_{1} \varepsilon_{2} \beta_{1} \beta_{2}}{\varepsilon_{2}^{2} \beta_{1}^{2}+\varepsilon_{1}^{2} \beta_{2}^{2}}
$$

The corresponding eigen-vectors are still $\left(A_{1}^{1}, C_{1}^{1}\right)=\left(1,-k_{y} / k_{z}\right)$ and $\left(A_{1}^{2}, C_{1}^{2}\right)=\left(k_{y} / k_{z}, 1\right)$, respectively. We have designated these two modes as mode 1 and mode 2. Eqs. (2.99) and (2.100) are both complex transcendent equations with an infinite number of roots in the complex plane, which need to be numerically solved by searching the whole complex plane. To avoid this numerical difficulty, we start from solutions for a perfectconducting metal wall. We keep in mind that with a large number of $\varepsilon_{2}$ in the mid-infrared regime, the eigenmodes within the grating should not depart far away from those within the corresponding perfect-conducting structure, which are known in the above sections. Therefore, we can use simple iteration techniques to find the accurate solutions of $\beta_{1}$ in Eqs. (2.99) and (2.100). For higher modes $m \geq 1$, we set the initial value of $\beta_{1}$ to be $\beta_{1}^{(0)}=s_{m}$, then the following iteration algorithm is followed:

$$
\operatorname{tg}\left[\beta_{1}^{(n+1)} a\right]=\frac{-2 i \beta_{1}^{(n)} \beta_{2}^{(n)}}{\left[\beta_{1}^{(n)}\right]^{2}+\left[\beta_{2}^{(n)}\right]^{2}}
$$




$$
\operatorname{tg}\left[\beta_{1}^{(n+1)} a\right]=\frac{-2 i \varepsilon_{1} \varepsilon_{2} \beta_{1}^{(n)} \beta_{2}^{(n)}}{\varepsilon_{2}^{2}\left[\beta_{1}^{(n)}\right]^{2}+\varepsilon_{1}^{2}\left[\beta_{2}^{(n)}\right]^{2}}
$$

where $n=0,1,2, \cdots,\left[\beta_{2}^{(n)}\right]^{2}=\left(\varepsilon_{2}-\varepsilon_{1}\right) k_{0}^{2}+\left[\beta_{1}^{(n)}\right]^{2}$. In practice, several iteration loops are enough to bring us to a convergent solution of $\beta_{1}$ with considerable accuracy.

The iteration technique can not be applied to the lowest mode by starting from $\beta_{1}^{(0)}=s_{0}=0$. It is easy to find that $\beta_{1}=0$ is a solution of both Eqs. (2.99) and (2.100). However, it can be shown that this solution is unphysical unless $\varepsilon_{2}$ is infinite. Since Eq. (2.99) has only solution of $\beta_{1}=0$, it is excluded. Noticing that $\beta_{1}$ is a small number, we find from Eq. (2.100) as an approximation the following formula:

$$
\beta_{1} a=\frac{-2 i \varepsilon_{1} \varepsilon_{2} \beta_{1} \beta_{2}}{\varepsilon_{2}^{2} \beta_{1}^{2}+\varepsilon_{1}^{2} \beta_{2}^{2}}
$$

whose non-zero solution is $\beta_{1}=\left\{-\left[a \varepsilon_{1}^{2}\left(\beta_{2}^{(0)}\right)^{2}+2 i \beta_{2}^{(0)} \varepsilon_{1} \varepsilon_{2}\right] / a \varepsilon_{2}^{2}\right\}^{1 / 2}$, where $\beta_{2}^{(0)}=\left(\varepsilon_{2}-\varepsilon_{1}\right)^{1 / 2} k_{0}$. To improve the accuracy of solution, we can do search in a small region on the complex plane around $\beta_{1}=\left\{-\left[a \varepsilon_{1}^{2}\left(\beta_{2}^{(0)}\right)^{2}+2 i \beta_{2}^{(0)} \varepsilon_{1} \varepsilon_{2}\right] / a \varepsilon_{2}^{2}\right\}^{1 / 2}$.

What happens if $\varepsilon_{2}$ is infinite? Obviously, for the lowest mode, $\beta_{1} \rightarrow 0$. For higher modes, we have double-degenerate solutions as $\operatorname{tg}\left(\beta_{1} a\right)=0$, which yields $\beta_{m}=m \pi / a=s_{m}, m \geq 1$. The eigenvectors are the same as in the finite-conducting situations $\left(A_{1}^{1}, C_{1}^{1}\right)$ and $\left(A_{1}^{2}, C_{1}^{2}\right)$. However, the amplitudes in the metal domain $A_{2}, B_{2}, C_{2}, D_{2}$ are all zero, due to the infinitely large $T_{2}$ matrix. Since these two modes are double-degenerate, we 
can reorganize the eigenvectors such that $\left(A_{1}, C_{1}\right)=(1,0)$ and $\left(A_{1}, C_{1}\right)=(0,1)$. This we see has returned to the solution for a perfect-conducting grating. For the lowest mode where $\beta_{1}=0$, the eigenvector is still $\left(A_{1}^{2}, C_{1}^{2}\right)$. However, from Eq. (4.3) $E_{x}$ has infinite large amplitude. So, if we set the amplitude of $E_{x}$ to unity, the amplitude of $E_{y}\left(A_{1}\right)$ and $E_{z}\left(C_{1}\right)$ are both zero in effect. Therefore, in this case, we also return to the solution of eigenmode in a perfectconducting grating.

Now we can write down the EM fields inside the grating region using eigenmode expansions. The tangential field components are

$$
\begin{aligned}
& E_{x}(\mathbf{r})=\sum_{m}\left[A_{m} e^{i k_{z, m 1^{*}}^{+}} X_{m 1}^{+}(x)+B_{m} e^{i k_{z, m 1}^{-} z} X_{m 1}^{-}(x)\right. \\
& \left.+C_{m} e^{i k_{z, m 2}^{+} z} X_{m 2}^{+}(x)+D_{m} e^{i k_{z, m 2^{z}}^{-}} X_{m 2}^{-}(x)\right] e^{i k_{y} y}, \\
& E_{y}(\mathbf{r})=\sum_{m}\left[A_{m} e^{i k_{z, m 1}^{+} z} Y_{m 1}^{+}(x)+B_{m} e^{i k_{z, m 1}^{-} z^{2}} Y_{m 1}^{-}(x)\right. \\
& \left.+C_{m} e^{i k_{z, m}^{+} z} Y_{m 2}^{+}(x)+D_{m} e^{i k_{z, m 2}^{-} z} Y_{m 2}^{-}(x)\right] e^{i k_{y} y}, \\
& H_{x}(\mathbf{r})=\sum_{m}\left[A_{m} e^{i k_{z, m 1}^{+} z} U_{m 1}^{+}(x)+B_{m} e^{i k_{z, m n}^{-} z} U_{m 1}^{-}(x)\right. \\
& \left.+C_{m} e^{i k_{s, m 2}^{+} z} U_{m 2}^{+}(x)+D_{m} e^{i k_{z, m 2}^{-} z} U_{m 2}^{-}(x)\right] e^{i k_{y} y},
\end{aligned}
$$




$$
\begin{gathered}
H_{y}(\mathbf{r})=\sum_{m}\left[A_{m} e^{i k_{s, 11}^{+} z} V_{m 1}^{+}(x)+B_{m} e^{i k_{s m 1}^{-} z} V_{m 1}^{-}(x)\right. \\
\left.+C_{m} e^{i k_{s, m}^{+} z} V_{m 2}^{+}(x)+D_{m} e^{i k_{z, m}^{m} z^{2}} V_{m 2}^{-}(x)\right] e^{i k_{y} y}
\end{gathered}
$$

Here $X_{m 1}^{+}(x)$ is the modal function of the E-field $x$-component connected to mode 1 under the upwards $k_{z}$ wave vector $k_{z, m 1}^{+}$, see the definition in the bracket "[ ]" in Eq. (2.73) and Eq. (2.77). Others are similarly defined. $A_{m}, B_{m}, C_{m}, D_{m}$ are modal coefficients. For the lowest mode $m=0$, since only mode 2 is present, $A_{m}$ and $B_{m}$ vanish.

To solve the scattering of a plane EM wave by the 1D metallic lamellar grating, we use the boundary conditions at the two interfaces of $z=h$ and $z=0$. We also use the method of moment similar to that employed in the case of a perfect-conducting grating. We project the E-field onto the plane wave basis and obtain

$$
\begin{aligned}
& C_{0} e^{i k_{, 202}^{+} h} I_{i, 0}^{(3)}+D_{0} e^{i k k_{2,2}^{-} h} I_{i, 0}^{(4)}+\sum_{m \geq 1}\left[A_{m} e^{i k_{z, m}^{\dagger} h} I_{i, m}^{(1)}\right. \\
& \left.+B_{m} e^{i k_{z, m}^{-} h} I_{i, m}^{(2)}+C_{m} e^{i k_{z, m 2}^{+}{ }^{h}} I_{i, m}^{(3)}+D_{m} e^{i k_{z, m}^{-} z^{h}} I_{i, m}^{(4)}\right]=d E_{x i}(h), \\
& C_{0} e^{i k k_{, 02}^{+} h} J_{i, 0}^{(3)}+D_{0} e^{i k z_{2,2 h}^{*} h} J_{i, 0}^{(4)}+\sum_{m \geq 1}\left[A_{m} e^{i k k_{m, m}^{\dagger} h} J_{i, m}^{(1)}\right.
\end{aligned}
$$




$$
\begin{gathered}
\left.+B_{m} e^{i k_{z, m}^{-} h^{h}} J_{i, m}^{(2)}+C_{m} e^{i k_{i, m}^{\prime} h} J_{i, m}^{(3)}+D_{m} e^{i k_{z, m} h^{h}} J_{i, m}^{(4)}\right]=d E_{y i}(h) \\
C_{0} I_{i, 0}^{(3)}+D_{0} I_{i, 0}^{(4)}+\sum_{m \geq 1}\left[A_{m} I_{i, m}^{(1)}+B_{m} I_{i, m}^{(2)}+C_{m} I_{i, m}^{(3)}+D_{m} I_{i, m}^{(4)}\right]=d E_{x i}(0) \\
C_{0} J_{i, 0}^{(3)}+D_{0} J_{i, 0}^{(4)}+\sum_{m \geq 1}\left[A_{m} J_{i, m}^{(1)}+B_{m} J_{i, m}^{(2)}+C_{m} J_{i, m}^{(3)}+D_{m} J_{i, m}^{(4)}\right]=d E_{y i}(0)
\end{gathered}
$$

Here the moment between a plane wave function and a modal function is defined as

$$
\begin{aligned}
& I_{i, m}^{(1)}=\int_{0}^{d} e^{-i k_{x x} x} X_{m 1}^{+}(x) d x, \quad I_{i, m}^{(2)}=\int_{0}^{d} e^{-i k_{x \rightarrow} x} X_{m 1}^{-}(x) d x, \\
& I_{i, m}^{(3)}=\int_{0}^{d} e^{-i k_{x} x} X_{m 2}^{+}(x) d x, \quad I_{i, m}^{(4)}=\int_{0}^{d} e^{-i k_{x} x} X_{m 2}^{-}(x) d x .
\end{aligned}
$$

$J_{i, m}^{(k)}, M_{i, m}^{(k)}$, and $N_{i, m}^{(k)}(k=1,2,3,4)$ are obtained by replacing $X(x)$ by $Y(x), U(x)$, and $V(x)$ in the integration, respectively.

The boundary condition for the $\mathrm{H}$-field is matched by projecting the $H_{x}$ field onto the modal functions of mode 1 , while projecting the $H_{y}$ field onto the modal functions of mode 2. This results in

$$
C_{0} e^{i k_{z, 02}^{+} h} S_{m, 0}^{(3)}+D_{0} e^{i k_{z, 02}^{-} h} S_{m, 0}^{(4)}+\sum_{m^{\prime} \geq 1}\left[A_{m^{\prime}} e^{i k_{s, m^{\prime}}^{+} h} S_{m, m^{\prime}}^{(1)}\right.
$$




$$
\left.+B_{m^{\prime}} e^{i k_{z, m^{\prime} 1^{-} h}} S_{m, m^{\prime}}^{(2)}+C_{m m^{\prime}} e^{i k_{z, m 2^{\prime} h}^{+} h} S_{m, m^{\prime}}^{(3)}+D_{m^{\prime}} e^{i k_{z, m 2^{\prime}}^{-} h} S_{m, m^{\prime}}^{(4)}\right]=\sum_{i} H_{x i}(h) M_{-i, m}^{(1)}
$$

$m=1,2, \cdots$

$$
\begin{gathered}
C_{0} e^{i k_{z, 02}^{+} h} T_{m, 0}^{(3)}+D_{0} e^{i k_{z, 02^{2} h}^{-} h} T_{m, 0}^{(4)}+\sum_{m^{\prime} \geq 1}\left[A_{m m^{\prime}} e^{i k_{z, m^{\prime}}^{+} h} T_{m, m^{\prime}}^{(1)}\right. \\
\left.+B_{m^{\prime}} e^{i k_{z, m^{\prime}}^{-} h} T_{m, m^{\prime}}^{(2)}+C_{m^{\prime}} e^{i k_{z, m}^{+} h} T_{m, m^{\prime}}^{(3)}+D_{m^{\prime}} e^{i k_{z, m 2^{\prime} h}^{h}} T_{m, m^{\prime}}^{(4)}\right]=\sum_{i} H_{y i}(h) N_{-i, m}^{(3)},
\end{gathered}
$$

$m=0,1,2, \cdots$,

$$
C_{0} S_{m, 0}^{(3)}+D_{0} S_{m, 0}^{(4)}+\sum_{m^{\prime} \geq 1}\left[A_{m^{\prime}} S_{m, m^{\prime}}^{(1)}+B_{m^{\prime}} S_{m, m^{\prime}}^{(2)}+C_{m^{\prime}} S_{m, m^{\prime}}^{(3)}+D_{m^{\prime}} S_{m, m^{\prime}}^{(4)}\right]=\sum_{i} H_{x i}(0) M_{-i, m}^{(1)}
$$

$m=1,2, \cdots$,

$$
C_{0} T_{m, 0}^{(3)}+D_{0} T_{m, 0}^{(4)}+\sum_{m^{\prime} \geq 1}\left[A_{m} T_{m, m^{\prime}}^{(1)}+B_{m} T_{m, m^{\prime}}^{(2)}+C_{m} T_{m, m^{\prime}}^{(3)}+D_{m^{\prime}} T_{m, m^{\prime}}^{(4)}\right]=\sum_{i} H_{y i}(0) N_{-i, m}^{(3)},
$$

$m=0,1,2, \cdots$. The moment between two modal functions is defined as

$$
S_{m, m^{\prime}}^{(1)}=\int_{0}^{d} U_{m 1}^{+}(x) U_{m^{\prime} 1}^{+}(x) d x, \quad S_{m, m^{\prime}}^{(2)}=\int_{0}^{d} U_{m 1}^{+}(x) U_{m^{\prime} 1}^{-}(x) d x
$$




$$
\begin{gathered}
S_{m, m^{\prime}}^{(3)}=\int_{0}^{d} U_{m 1}^{+}(x) U_{m^{\prime} 2}^{+}(x) d x, \quad S_{m, m^{\prime}}^{(4)}=\int_{0}^{d} U_{m 1}^{+}(x) U_{m^{\prime} 2}^{-}(x) d x, \\
T_{m, m^{\prime}}^{(1)}=\int_{0}^{d} V_{m 2}^{+}(x) V_{m^{\prime} 1}^{+}(x) d x, \quad T_{m, m^{\prime}}^{(2)}=\int_{0}^{d} V_{m 2}^{+}(x) V_{m^{\prime} 1}^{-}(x) d x, \\
T_{m, m^{\prime}}^{(3)}=\int_{0}^{d} V_{m 2}^{+}(x) V_{m^{\prime} 2}^{+}(x) d x, \quad T_{m, m^{\prime}}^{(4)}=\int_{0}^{d} V_{m 2}^{+}(x) V_{m^{\prime} 2}^{-}(x) d x
\end{gathered}
$$

From Eqs. (2.108)-(2.115) we can delete the unknown variables for the modal amplitude, and obtain the following matrix equation that connect the E-field and $\mathrm{H}$-field in both sides of the grating:

$$
\left(\begin{array}{l}
E_{x i}(h) \\
E_{y i}(h) \\
E_{x i}(0) \\
E_{y i}(0)
\end{array}\right)=\left(\begin{array}{llll}
P_{11} & P_{12} & P_{13} & P_{14} \\
P_{21} & P_{22} & P_{23} & P_{24} \\
P_{31} & P_{32} & P_{33} & P_{34} \\
P_{41} & P_{42} & P_{43} & P_{44}
\end{array}\right)\left(\begin{array}{l}
H_{x i}(h) \\
H_{y i}(h) \\
H_{x i}(0) \\
H_{y i}(0)
\end{array}\right)
$$

This directly lead to the $R$-matrix formula for the scattering of a single $k_{y}=k_{y j}$ component of plane wave by one single layer of the 2D layer-by-layer metallic grating:

$$
\left(\begin{array}{c}
\Omega_{0, j}^{+} \\
\Omega_{1, j}^{+}
\end{array}\right)=\left(\begin{array}{ll}
r_{11}^{(j)} & r_{12}^{(j)} \\
r_{21}^{(j)} & r_{22}^{(j)}
\end{array}\right)\left(\begin{array}{c}
\Omega_{0, j}^{-} \\
\Omega_{1, j}^{-}
\end{array}\right)
$$

For the whole $2 \mathrm{D}$ scattering problem, we directly write down

$$
\left(\begin{array}{c}
\Omega_{0}^{+} \\
\Omega_{1}^{+}
\end{array}\right)=\left(\begin{array}{ll}
r_{11} & r_{12} \\
r_{21} & r_{22}
\end{array}\right)\left(\begin{array}{l}
\Omega_{0}^{-} \\
\Omega_{1}^{-}
\end{array}\right),
$$


This can yield the transmission, reflection, and absorption spectra under an arbitrary plane EM wave incidence. The transmission $T$ and reflection $R$ are calculated by means of Eqs. (2.33) and (2.34). The absorption is calculated according to $A=1-T-R$. 


\section{CHAPTER 3. Photonic Band Gaps of Conformally Coated Structures}

In this chapter we will show that conformal coatings on a dielectric photonic structure can modify its optical properties. The metallic coating on a dielectric structure not only leads to a much larger photonic band-gap, but also shifts the band-edge to shorter wavelength. The high refractive index dielectric coating on a polymeric molds which has low dielectric constant, preventing formation of a photonic bandgap shows the possibility of new way to realize photonic crystals.

\subsection{Introduction}

There is a great deal of current interest in all-metallic three-dimensional (3D) photonic lattices for energy applications $[5,19]$. At the near infrared wavelengths $(\lambda=1-2 \mu \mathrm{m})$, it is suggested that the use of a metallic lattice could lead to an efficient electricity generation [19]. At the visible wavelengths, a photonic-lattice filament has the potential to achieve more than $50 \%$ electric-to-optical efficiency [20]. To realize these devices, one of the main challenges is in the fabrication of photonic-lattice at a large scale and small feature sizes $(<100-400 \mathrm{~nm})$ [21-28]. Another challenge is in choosing a suitable metal with a desirable

real, $\varepsilon_{\text {real }}$, and imaginary, $\varepsilon_{i}$, dielectric constants. Here, we propose a new way for altering 
optical properties of a 3D photonic-lattice using material-coating. The coating technique we propose is precise to nano-meter scale and the choices of metals are many, such as $\mathrm{Ni}, \mathrm{Pd}, \mathrm{Pt}$, $\mathrm{Co}, \mathrm{Ru}, \mathrm{Cu}$ and $\mathrm{Au}$. It is experimentally and theoretically demonstrated that a thin layer of metal-coating strongly affects the dispersion of a photonic lattice. The sample used in the experiment is a 3D silicon photonic-lattice structure. The photonic-lattice is constructed in a layer-by-layer fashion using 1D grating array and has diamond-lattice symmetry [28-30]. The lattice-constant is $a=650 \mathrm{~nm}$, the rod-width is targeted at $\mathrm{w}=180 \mathrm{~nm}$ and the filling fraction is $\sim 28 \%$.

Dielectric conformal coatings are very widely used in the microelectronics industry. Such a coating provides mechanical and environmental protection to extend the life of electronic components and circuitry, including corrosion resistance, electrical insulation and protection against short circuits. We also demonstrate that dielectric conformal coating techniques can considerably modify the optical properties of photonic structures[31].

Three-dimensional photonic crystals with complete band gaps for omni-directional propagation of electromagnetic waves have immense potential for devices in fiber-optics based telecommunications applications, single-mode waveguides, channel add-drop filters, catalysis and control of spontaneous emission. The three-dimensional woodpile structures with a large three dimensional photonic band gap have been fabricated with semiconductor fabrication methods. Such photonic crystals shows excellent optical performance over relatively small areas. It is desirable to investigate alternative economical fabrication methods that enable fabricate large area photonic crystals operating at optical and nearinfrared wavelengths. 
An potential fabrication method is micro-transfer mold method. The mold can be filled with high index dielectric materials or metals. Another ways to realize photonic crystals are coating of higher refractive index dielectric materials or metals In this chapter we will show the experimentally measured and theoretically calculated optical properties of coated structures.

\subsection{Coated Structures}

The metallic coating was accomplished firstly by chemical vapor deposition (CVD) of a thin Cobalt film as the seed layer from a metallorganic $\mathrm{Co}_{2}(\mathrm{CO})_{8}$ precursor [32]. It is then follow by the electro-less deposition (ELD) of a Copper layer [33]. In a typical ELD process, the $\mathrm{Cu}$ film is deposited via a redox process in a plating chemical bath. The $\mathrm{Cu}$

plating bath typically contains $\quad \mathrm{CuSO}_{4} \cdot 5 \mathrm{H}_{2} \mathrm{O} \quad(64 \mathrm{~g} / \mathrm{L}), \quad \mathrm{C}_{10} \mathrm{H}_{16} \mathrm{~N}_{2} \mathrm{O}_{8}$ (ethylenediaminetetraacetic acid, EDTA, $70.0 \mathrm{~g} / \mathrm{L})$, glyoxylic acid $(18.0 \mathrm{~g} / \mathrm{L})$, polyethylene glycol (PEG, $0.5 \mathrm{~g} / \mathrm{L}$ ), and tetramethylammonium hydroxide (TMAH) as a $\mathrm{pH}$ controller (adjusted to about 12.5). The bath temperature was maintained at a temperature of $70^{\circ} \mathrm{C}$. A schematic of the coating scheme is shown in Fig. 3.1 (a). A SEM image of a calibrated 1Dgrating structure is also shown in Fig.1 (b). The Cu thin film is conformal and has a thickness of $\sim 70 \mathrm{~nm}$.

The dielectric coating was accomplished by atomic layer deposition (ALD) of a thin titania film. An SEM image of the coated structure is shown in Fig. 3.2. We first synthesize a four layer polyurethane mold template with a bar separation of $2.5 \mu m$ that was easily 
achieved over a large area by the microtransfer mold method [34]. The layer-by-layer stacking of the template was achieved by alignment methods [35]. For these preliminary studies, we experimentally employed a moderate polyurethane bar width $w \sim 1.4 \mu$ and conformally coated the structure with titania to a coating thickness of $0.45 \mu$, using the atomic layer deposition method. Since the processing temperatures are $\sim 100{ }^{\circ} \mathrm{C}$, the titania remains in the anatase phase rather the higher index rutile phase. The resulting coated structure demonstrates a uniform coating of each rod including on the back-side of each rod, with rounded corners.

\subsection{Metallic Coating - Theoretical and Experimental Results}

The metal coated photonic crystal structure consists of silicon bars(width $=180 \mathrm{~nm}$, height $=220 \mathrm{~nm}$ ) conformally coated with cobalt and copper and its geometry is different from a normal layer by layer structure as shown in Fig. 3.3 (a). To calculate this we used transfer matrix method calculation in real space for the actual cobalt-copper coated lattice.

Each layer of our structure can be decomposed into sub-layers in which the structure simplifies to a one-dimensional grating. Within each such grating layer, Maxwell's equations can be rigorously solved in a plane wave basis set. The simulations utilize the experimental values of $n_{1}+i n_{2}$ for silicon, cobalt and copper. The sub-layers are shown in Fig. 3.3 (b).

In Fig. 3.4, theoretical calculation results of reflectance spectra are shown for a fivelayer silicon (the blue curve) and copper (the red curve) photonic-lattice, respectively. The 
overall rod width of the copper photonic-lattice is $140 \mathrm{~nm}$ wider than that of the silicon-rod, due to a copper over-coating of $\sim 70 \mathrm{~nm}$.

The $3 \mathrm{D}$ silicon sample shows a high reflectance at the $\lambda \sim 1.6-1.9 \mu m$ spectral range and a short wavelength band-edge at $\lambda \sim 1.5 \mu \mathrm{m}$. Clearly, this band-edge is far from the desirable visible regime of $\lambda \sim 400-700 \mathrm{~nm}$. On the other hand, the 3D copper photoniclattice sample exhibits a very different reflectance characteristic. The first photonic bandedge occurs at a much shorter wavelength, $\lambda \sim 700-800 \mathrm{~nm}$, even though the lattice dimensions are essentially the same. Also, the high reflectance extends into the mid-infrared wavelength. The weak reflectance dip around $\lambda \sim 1.3-1.7 \mu m$ is due to a slight absorption of the copper material. This calculation shows that, the $3 \mathrm{D}$ copper photonic-lattice is a better choice for realizing visible photonic band-gap structures. In Fig. 3.5 experimental reflectance spectra taken from a silicon photonic-lattice (the blue curve) and the Cu-coated lattice (the red curve) are shown, respectively. As predicted, the silicon lattice shows a high reflectance at $\lambda \sim 1.6-1.9 \mu \mathrm{m}$ and has a finite band-gap. Its upper and lower band-edges are at $\lambda_{\text {band-edge }} \sim 1.5 \mu \mathrm{m}$ and $\lambda_{\text {band-edge }} \sim 2.25 \mu \mathrm{m}$, respectively. For the Cu-coated sample, it shows a different reflectance spectrum. Its high reflectance regime extends from $\lambda \sim 0.7-0.8$ $\mu m$ into the infrared, consistent with that reported earlier for an all-metallic photonic-lattice [1]. More importantly, the band-edge is now shifted from $\lambda \sim 1.5 \mu \mathrm{m}$ for the silicon-lattice to $\lambda \sim 750 \mathrm{~nm}$ for the $\mathrm{Cu}$-coated lattice. The appearance of a photonic band-edge at a much shorter wavelength, or a higher frequency, is an important benefit of using the Cu-coated sample. It is also noted that the higher frequency oscillations at higher frequencies is not as 
prominent as that predict theoretically for both the silicon and copper photonic-crystal samples. It is suspected that this is due to light scattering loss by diffractions.

This data illustrates that a photonic band-edge at $\lambda \sim 750 \mathrm{~nm}$ is achievable and an efficient near-visible emission is feasible. We comment that more theoretical modeling and formulation is needed in the future to account for the effect of $\mathrm{Co} / \mathrm{Cu}$ composite coating. For thermal stability and mechanical strength considerations, it is also important to explore using other higher temperature materials, such as Rhodium and Ruthenium, to build photoniclattice.

\subsection{Dielectric Coating - Theoretical and Experimental Results}

The optical data of the dielectric coated structure were measured with an FTIR spectrometer and compared to the uncoated polyurethane mold as shown in Fig. 3.6. There is transmission between $2-4.5 \mu \mathrm{m}$ for both the mold and coated structure accompanied by significant reflection $(\mathrm{R})$ for the coated structure. For wavelengths above $5 \mu \mathrm{m}$ the transmission $(\mathrm{T})$ for both structures is very low since the substrate (Corning \#1 glass) absorbs strongly in this long-wavelength range. The glass absorption causes the transmission edge at $5 \mu m$. At short wavelengths below $4 \mu m$, substantial diffraction occurs causing the specular transmission to be low. After accounting for diffraction we find the absorption is low for wavelengths below $4 \mu \mathrm{m}$. The reflection peaks at $9 \mu \mathrm{m}$ and $11 \mu \mathrm{m}$ are present for the glass substrate and are the well known absorption peaks of silica from the glass where the $\operatorname{Im}(\varepsilon)$ has maxima. 
However the reflective peak between 6-7 $\mu m$ for the conformally coated structure is not present for the 4-layer mold. The position of this feature is consistent with the expected stop band in the (001) direction for such an over-coated structure, taking onto account the decrease of the refractive index of anatase titania to $\sim 1.9$ in this wavelength range.

This interpretation is supported by using transfer matrix method calculations performed on the multilayer structure as done for metal coated structure. The simulations utilize the experimental values of $(n, k)$ for titania and polyurethane. Experimental values of $(\mathrm{n}, \mathrm{k})$ for the glass substrate were inferred through measurement. Calculated reflection and transmission in Fig. 3.7 for the coated structure on a $100 \mu \mathrm{m}$ glass substrate shows transmission peaks near $3 \mu m$ and $4 \mu m$ in good agreement experiment, followed by very low transmission above $6 \mu m$, due to the absorption in glass. The calculated reflectance shows a weak peak between 6-7 $\mu \mathrm{m}$ in the same position as in the measurement. Calculated reflected peaks near $3 \mu m, 5 \mu m$, and $11 \mu m$ are in good agreement with data although there is some difference in the 9-10 $\mu m$ range between calculation and experiment. We interpret the reflective peak between 6-7 $\mu m$ that is accompanied by negligible transmission, as occurring from a weak photonic stop band in the stacking direction. This feature can be enhanced for higher refractive index contrasts or thinner coatings. Conformal coatings may improve the dielectric contrast in other low refractive index photonic crystals generated in photoresist by holographic methods [36] or direct laser writing [37]. 


\subsection{Conclusions}

In conclusion, a new approach is adapted to create a $3 \mathrm{D}$ metallic photonic-lattice with a band-edge near the visible wavelengths. The successful implementation of copper coating allows for a near visible 3D photonic band-edge of $\lambda \sim 750 \mathrm{~nm}$ with a complete band-gap and at a large scale. Also, dielectric conformal coatings can improve the optical contrast in low refractive index photonic structures fabricated by micro-transfer mold method. 


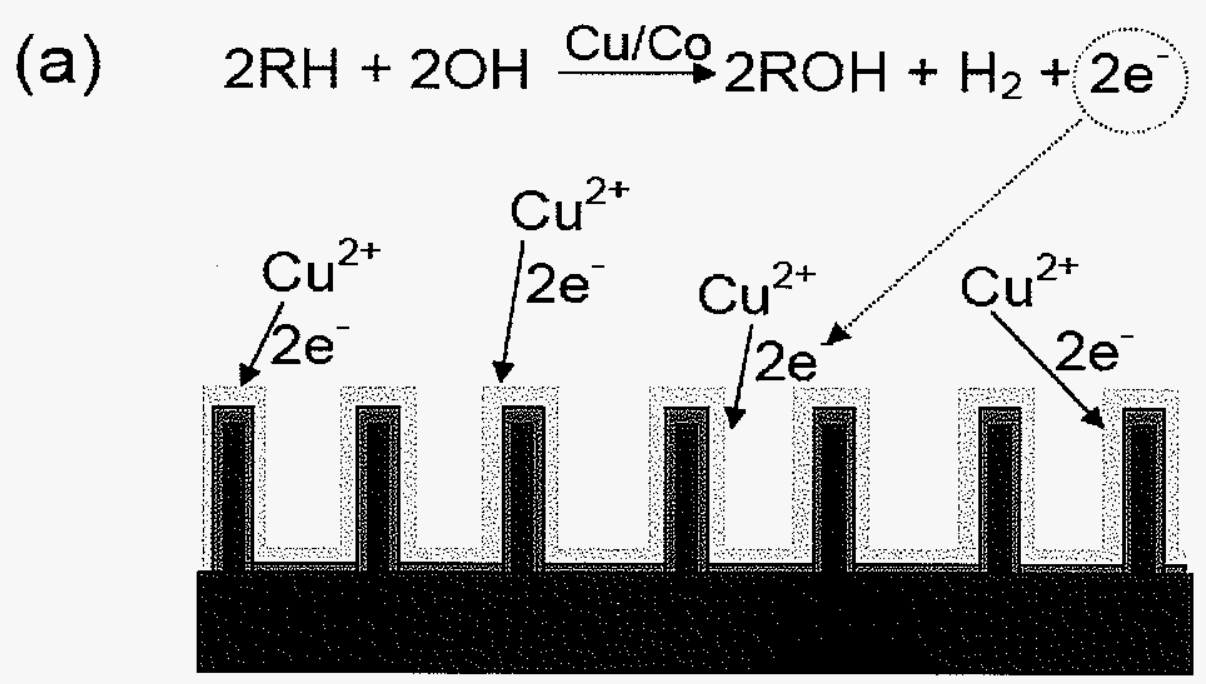

\section{$\mathrm{RH}=\mathrm{HCOCO}_{2} \mathrm{H}$ (Glyoxylic acid)}

(b)

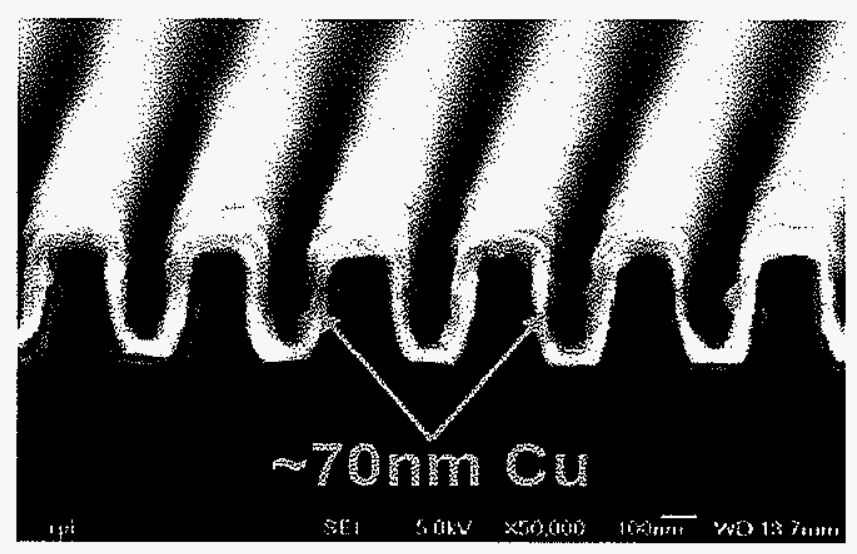

Fig. 3.1 (a) A schematic illustration of the proposed coating approach using CVD (chemical vapor deposition) and ELD (electro-less deposition) techniques. The green, orange and yellow colored areas represent the silicon substrate, the Co-seed-layer and the Cu-layer, respectively. (b) A SEM image of a calibrated sample-grating structure. The $\mathrm{Cu}$ thin film is quite conformal and has a thickness of $\sim 70 \mathrm{~nm}$. 


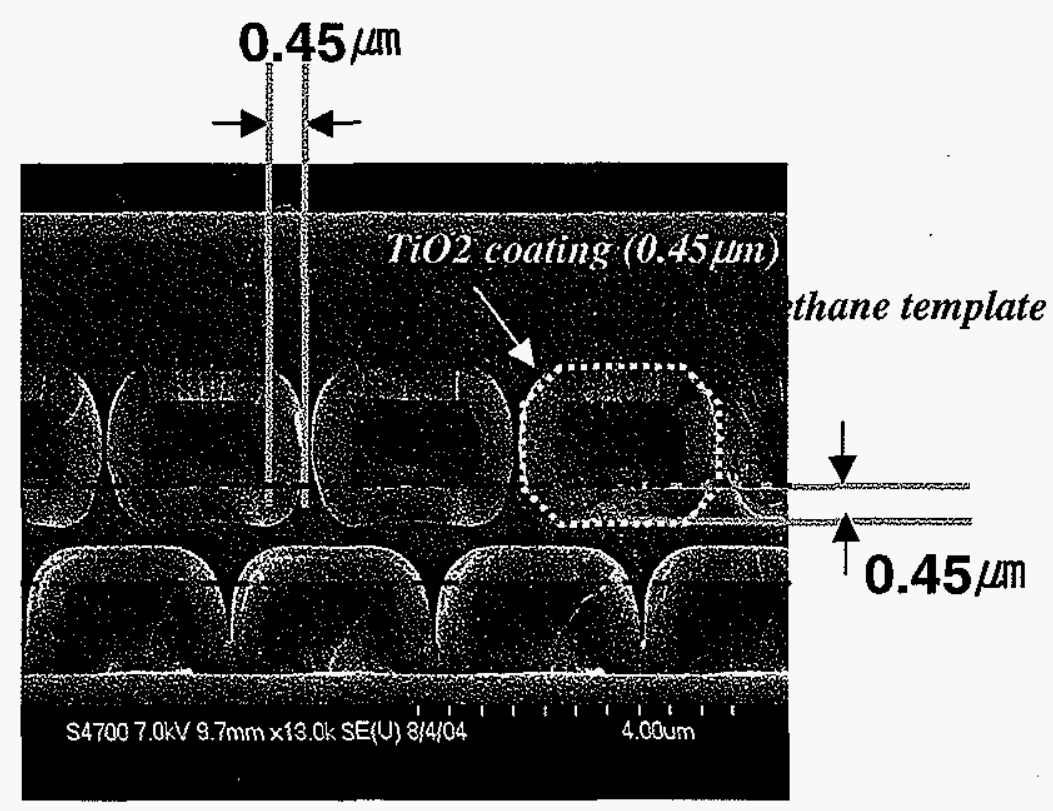

Fig. 3.2 Experimentally synthesized conformal titania coating of a four layer polyurethane bar template using the atomic layer deposition method. A titania coating of $0.45 \mu \mathrm{m}$ was achieved for a template with bar separation of $2.5 \mu \mathrm{m}$ and bar width $1.4 \mu \mathrm{m}$. 


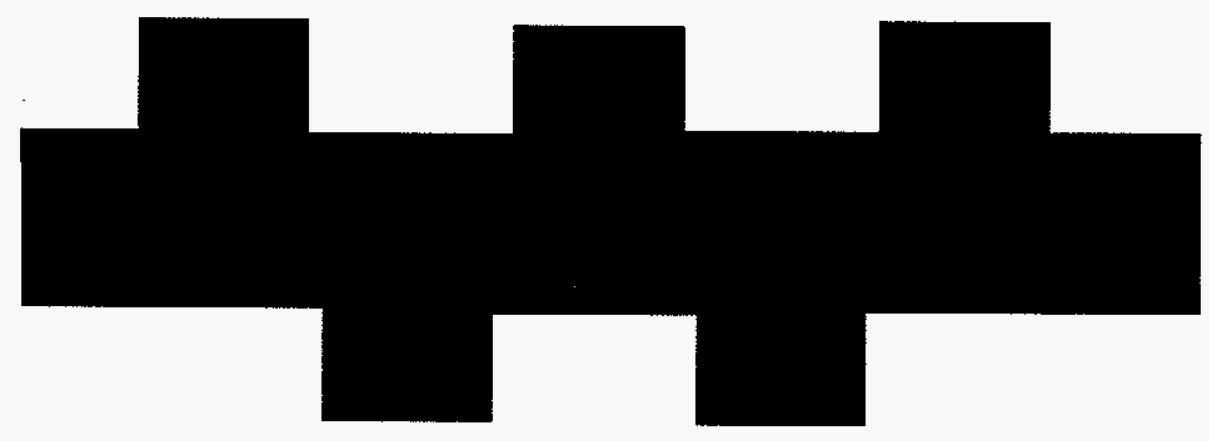

(a)

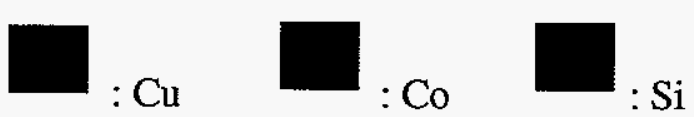

- || || ||

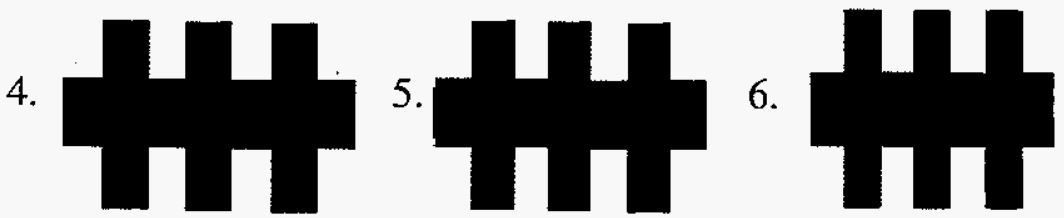

(b)

Fig 3.3 Configuration of metal coated structure. 


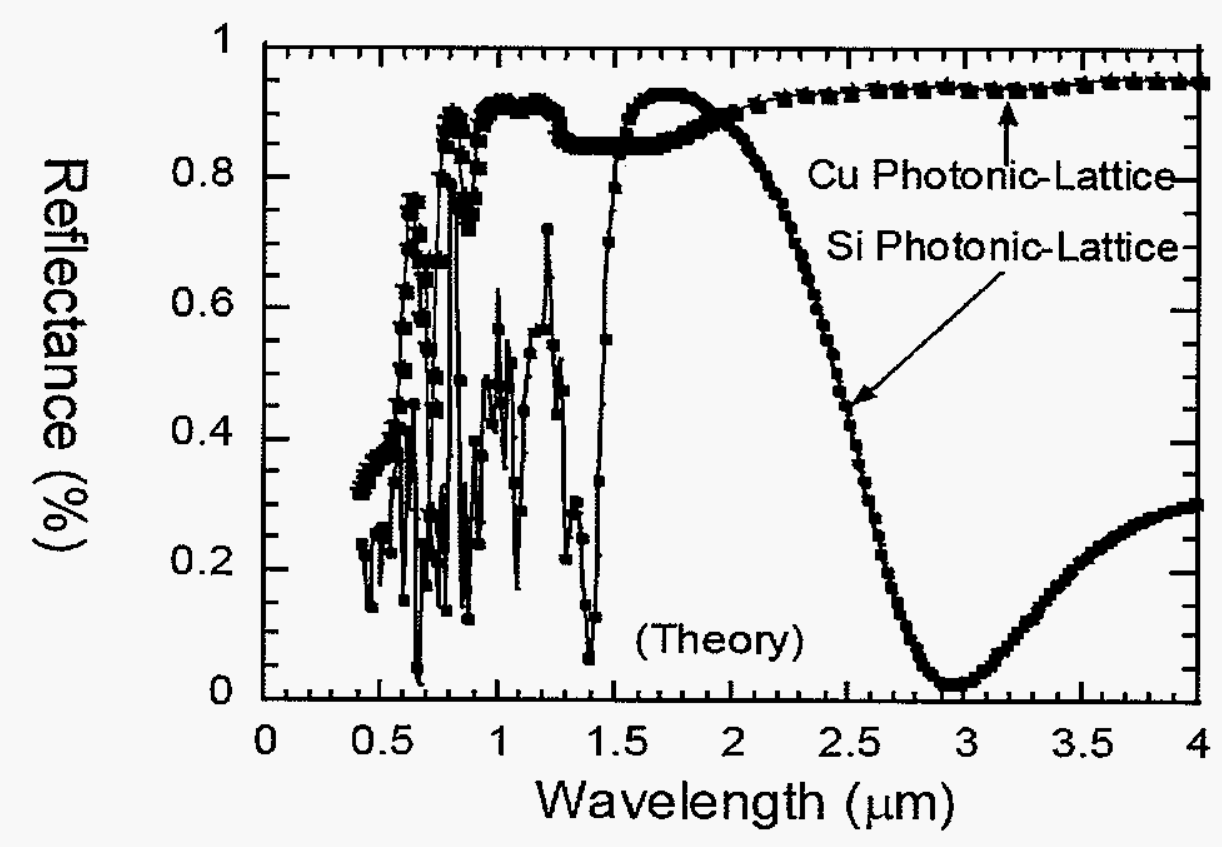

Fig. 3.4 Reflectance computed for a five-layer 3D silicon (the blue curve) and copper (the red curve) photonic-lattices, respectively. The 3D silicon lattice shows a high reflectance at the $\lambda$ $\approx 1.6-1.9 \mu \mathrm{m}$ spectral range and a band-edge at $\lambda \approx 1.5 \mu \mathrm{m}$. The 3D copper photonic-lattice exhibits a very different reflectance characteristic. Its first band-edge occurs at a much shorter wavelength, $\lambda \approx 700-800 \mathrm{~nm}$. 


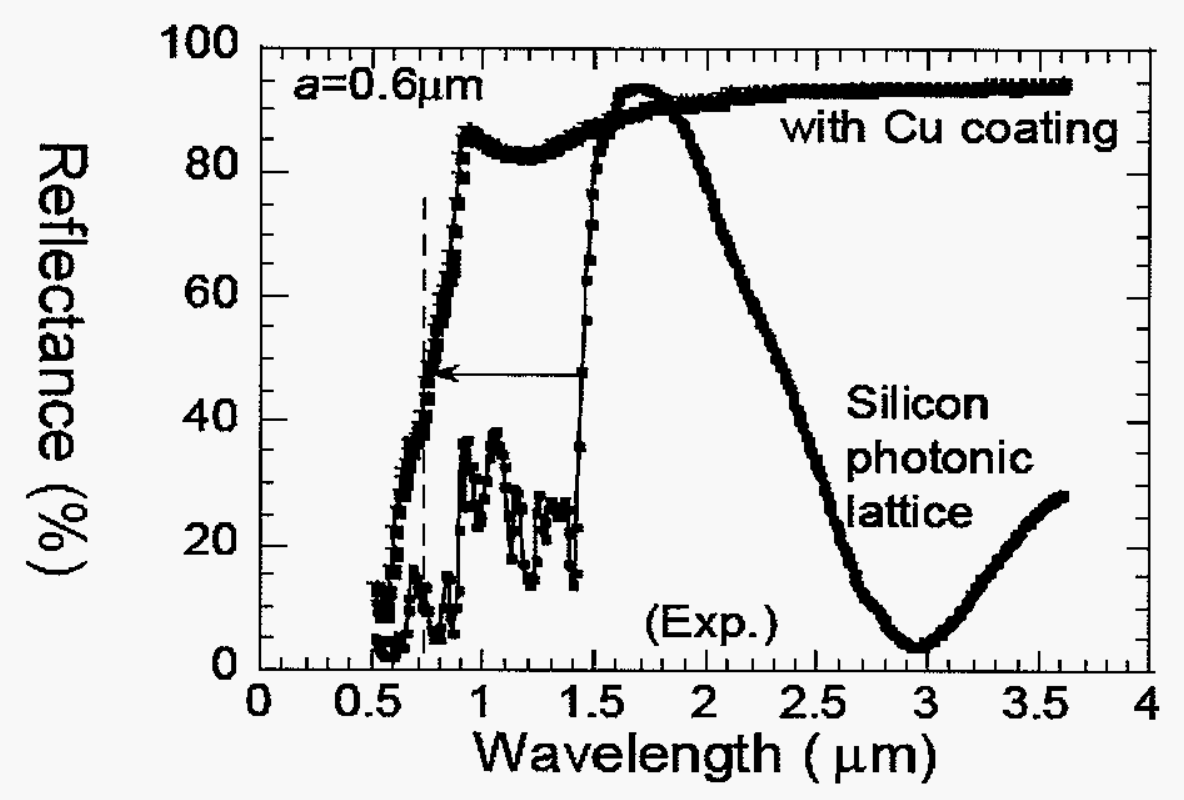

Fig. 3.5 Reflectance spectra taken from the silicon (black curve) and the Cu-coated (red curve) photonic-lattices. The silicon lattice shows a high reflectance at $\lambda \approx 1.7 \mu \mathrm{m}$ and a finite band-gap. On the other hand, The $\mathrm{Cu}$-coated sample shows a high reflectance regime extends from $\lambda \approx 0.9 \mu m$ into the infrared $(\lambda>4 \mu m)$. More importantly, the band-edge is shifted to a much shorter wavelength, $\lambda \approx 750 \mathrm{~nm}$, for the $\mathrm{Cu}$-coated sample. 

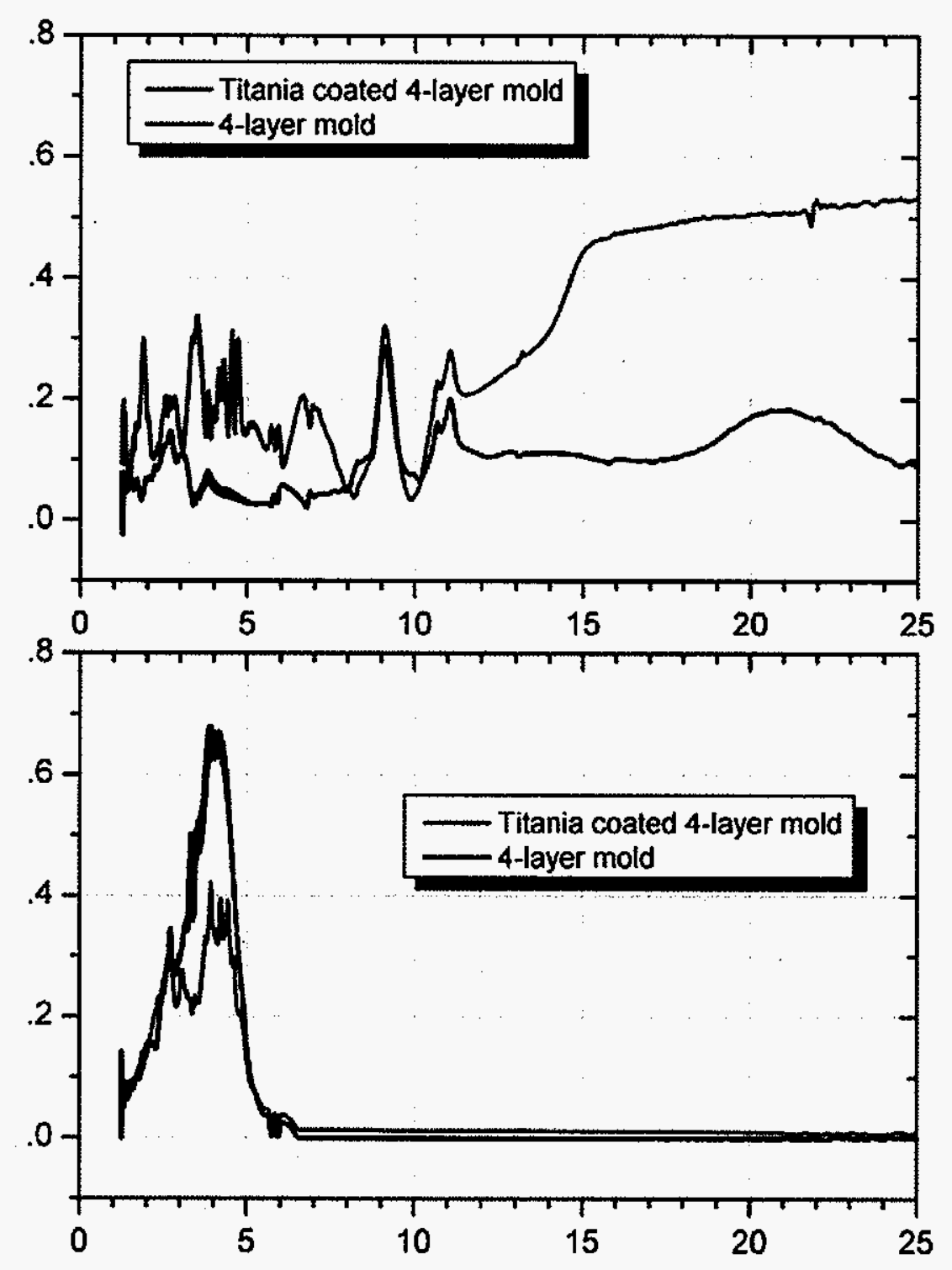

Fig. 3.6 Reflection and transmission measurements for the four-layer conformally coated structure on a $100 \mu \mathrm{m}$ thick glass substrate, compared to measurements for the uncoated polyurethane mold on the same glass substrate. 


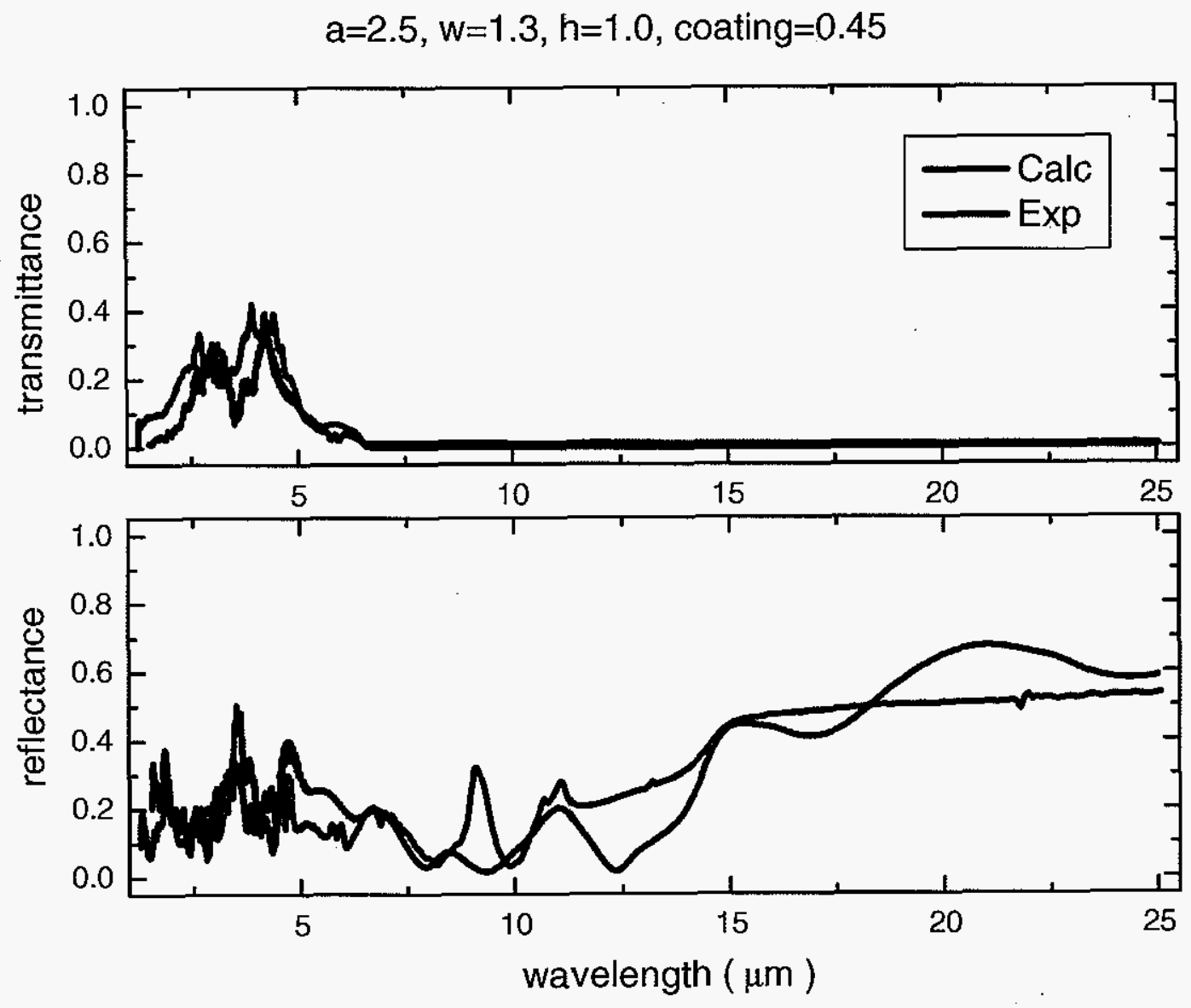

Fig. 3.7 Calculated reflectance and transmission. The calculations used a bar width $w=1.3$ $\mu m$, coating thickness $\mathrm{t}=0.45 \mu \mathrm{m}$, and pitch $\mathrm{a}=2.5 \mu \mathrm{m}$. 


\section{CHAPTER 4. Thermal Emission from Metallic Photonic Crystals}

In this chapter we will show how metallic photonic crystals modify and enhance absorption and how to relate absorption to emission. We will also discuss scaling-like behavior of metallic photonic crystals to choose a proper material and its limitation. Finally, we will show structural dependency of emission.

\subsection{Introduction}

It has been suggested that a metallic photonic crystal (MPC) structure may modify a thermal emission spectrum[4,5]. The experiment was carried out by Lin et al. by using a three dimensional woodpile structure made of tungsten[6]. The resulting thermal emission was shown to be suppressed in the photonic band gap and enhanced near the band edge. Promising experimental data have demonstrated that these metallic microstructures can be useful for incandescent lamp application and for thermal photovoltaic power generation.

Theoretical analysis has shown that MPC's can lead to significant enhancement of thermal radiation at a narrow frequency window around the band gap [38-41]. It has been well known that metals in their bulk material form exhibit small absorption in the midinfrared wavelength regime. For instance, a tungsten thin film has an absorption coefficient below $2 \%$ at around $4 \mu \mathrm{m}$. However, when metallic materials are brought into the form of 
microstructures such as 3D woodpile structure, there can be order-of-magnitude enhancement of absorption due to these microstructures [38-40].

The basic structural configurations of usual 3D woodpile MPC's are schematically depicted in Fig. 4.1. The MPC is stacked from rectangular metallic rods which are arrayed in a face-centered-tetragonal lattice. The stacking direction is set to be the (001) direction, which is parallel to the $z$ axis. This metallic structure can be built by infiltration of metallic materials into an original template, which is also a woodpile photonic crystal but made from dielectric materials such as silicon or polymer $[5,35]$.

In this chapter we will show how MPC modifies and enhances absorption and how to relate absorption to emission. We will also discuss scaling-like behavior of MPC and its limitation. Finally, we will show structural dependency of emission.

\subsection{Theories}

The emission from a hot body is defined at a $\lambda$ as

$$
a b s(\lambda, T) u(\lambda, T)
$$

where $a b s(\lambda, T)$ is the absorption and $u(\lambda, T)$ is Planck's blackbody emission at a wavelength $\lambda$ and temperature $T$, respectively. With this at hand we will discuss emission in the rest of this chapter. 
We first calculate the absorption spectrum of a woodpile tungsten MPC sample which has $a=2.8 \mu \mathrm{m}, w=0.85 \mu \mathrm{m}, h=0.9 \mu \mathrm{m}$ and 5 layers, where $a$ is pitch, $w$ is bar width and $h$ is height of the MPC. This MPC has been discussed experimentally[6]. The basic theoretical tool to solve this is the plane-wave-based transfer-matrix method in combination with the analytic modal solution approach. In brief, one first calculates the solution of the EM eigenmodes within each metallic layer by means of an analytical manipulation. Such an analytical step proves to be the key to the high efficiency of the whole theoretical scheme as the eigenmodes have already accurately accounted for the small skin depth of metal, which is two orders of magnitude smaller than the incident wavelength. The next step is to project these eigenmodes onto the plane-wave function space associated with the eigenmodes of EM fields in an air background. From this the transfer matrix for the metallic layer on the planewave basis can be built. The same procedure holds for all the five metallic layers in each unit cell, but a simpler and more efficient way is to utilize the translational and rotational symmetries between these layers. More details of the theoretical approaches can be referred to Refs. [40].

The calculation results are plotted in Fig. 4.2 (a). Here we have considered normal incidence of unpolarized plane waves. To achieve this, we consider incidence of two independent plane waves that are orthogonally polarized (one with the electric field parallel to the rod in the top layer and the other perpendicular) and have equal intensity. The resulting emission spectra derived with Eq. (4.1) are shown in Fig. 4.2 (b). The experimental results shows three distinct emission peak at $\lambda=3.5,4$, and $4.5 \mu \mathrm{m}$ and the calculation results also clearly show peaks at $\lambda=3.5,4 \mu \mathrm{m}$ except a peak at $\lambda=4.5 \mu \mathrm{m}$. In 
our calculations, no effect of disorders and surface roughness of metal rods are considered. This may account for the difference between theory and experiment.

\subsection{Results and Discussion}

We will first discuss how to choose MPC's base material in the interesting wavelength region by looking at its scaling-like behavior.

There has been great interest in realizing MPC's to modify a thermal emission spectrum either at the near infrared or visible wavelengths because of its potential application such as thermal photovoltaic cells for electric-power generation. Very recently, there was a challenge to achieve a three-dimensional metallic photonic crystal working at these wavelength regions by using tungsten as a base material[19]. Although metals like tungsten are high-loss materials, MPC's show scaling-like behavior until their band-edge pinned at a certain wavelength. It might be due to the intrinsic surface absorption of metal. To examine this we compared experimental results[42] and theoretical calculation results.

In Fig 4.3 (a) reflectance spectra taken from a series of 3D tungsten photonic-crystals of different lattice-constant are shown. In the inset, a representative SEM image of the MPC is also shown. The data is intended to illustrate the intrinsic material limitation of a tungstenphotonic-lattice at shorter wavelengths $(\lambda \leq 2 \mu m$ ). All five spectra exhibit a high reflectance plateau at longer wavelengths in the infrared. The reflectance also shows a transition from high to low at shorter wavelengths. As ao is decreased from 5, 2.8 to $1.5 \mu \mathrm{m}$, the photonic band-edge (at $50 \%$ reflectance value) decreased from $\lambda \sim 7,4.5$ to $2 \mu \mathrm{m}$ 
systematically. This behavior is consistent with the scaling rule of dielectric photonic crystals which don't have intrinsic absorption. However, as $a$ continues to decrease from $1.5,1.05 \mu \mathrm{m}$ to $600 \mathrm{~nm}$, the observed band-edge stays essentially at the same wavelength, $\lambda \sim 1.5-2 \mu \mathrm{m}$. Thus, at these wavelengths, the linearly scaling rule is no longer valid for tungsten photoniclattice samples which means that a tungsten photonic-lattice is not capable of yielding a fundamental band-edge either at the near-infrared or visible wavelengths. The calculation results are shown in Fig. 4.3 (b). It also shows the band-edge is pinned at $\lambda \sim 1.5-2 \mu \mathrm{m}$.

To understand this behavior, we calculated the absorption spectrum of tungsten thin film. The calculated absorption spectrum of tungsten is shown in Fig. 4.4 (a). As expected, the absorption of tungsten starts to increase rapidly at $\lambda \sim 2 \mu \mathrm{m}$ and it is about $10 \%$ at around that wavelength. It means that the intrinsic absorption of tungsten prevents the scaling down of the band-edge of tungsten photonic lattices. We examined several metals, such as gold, silver and copper by performing the same calculations. The calculated results show that the absorption of copper and gold start to increase rapidly at $\lambda \sim 600 \mathrm{~nm}$ and $540 \mathrm{~nm}$, respectively and that of silver is at $\lambda \sim 430 \mathrm{~nm}$. Therefore, they might be used for visible region. As an example we present gold 4-layer sample in Fig. 4.4 (b). In Fig. 4.5 we present the calculated optical data of several materials with pitch $=1.2 \mu \mathrm{m}$, bar width $=0.4 \mu \mathrm{m}$ and bar height $=0.5 \mu m$. They shows how base materials change MPC's optical properties.

Now, let us go forward to the structural dependency of woodpile MPC. To see the structural dependency of woodpile MPC, we calculated the optical properties of gold woodpile photonic crystals by changing the number of layers, bar height and filling fraction. 
First, we calculated the number of layer dependency of gold MPC with pitch $=300$ $\mathrm{nm}$, bar width $=100 \mathrm{~nm}$, height $=160 \mathrm{~nm}$. The number of layers are 2, 3, 4, 8 as presented in Fig. 4.6 (a) - (d). The absorption enhancement starts to appear at around $700 \mathrm{~nm}$ when the number of layers is 3 and when it is 8 as shown in Fig. 4.6 (d), the absorption peak is split into three peaks. This implies the coupling between different layers plays a role, although the stop band gap can be roughly explained by the waveguide cutoff wavelength. These characteristics are clear signals of strong PBG effect as explained in the paper by Li et al [38].

To see how the thickness of a bar changes absorption, we used gold 4-layer woodpile structure with varying the height from $85 \mathrm{~nm}$ to $145 \mathrm{~nm}$ as shown in Fig. 4.7. When the height is $85 \mathrm{~nm}$, absorption is not enhanced but when it become $105 \mathrm{~nm}$, the absorption enhancement starts to appear. It is because the absorption peak is red-shifting as the height goes thicker. As shown in Fig. 4.7, the absorption peak is shifting to longer wavelength, from $600 \mathrm{~nm}$, to $650 \mathrm{~nm}$, and $670 \mathrm{~nm}$, as height becomes thicker from $105 \mathrm{~nm}$ to $125 \mathrm{~nm}$ and 145 $n m$, respectively.

As another example of absorption enhancement, let us discuss metallic mesh structures which is shown in Fig 4.1 (c). To analyze this structure let us first discuss surface plasmon effect and waveguide cut-off wavelength.

As well-known[ 43 ], in a metallic grating, surface plasmon appears at $\lambda_{s p}=(a / m)\left[\operatorname{Re}\left\{[\varepsilon /(1+\varepsilon)]^{1 / 2}\right\} \pm \sin \theta\right]$, where $a$ is the pitch of a grating, $m$ is a nonzero positive integer, $\varepsilon$ is the dielectric constant of metal, and $\theta$ is the incident angle. For normal incidence and large $\varepsilon, \lambda_{s p}=a / m=2.5,1.25 \mu m$, etc for $a=2.5 \mu m$. 
It is also well-known that a square metallic waveguide has its cut-off wavelength at $\lambda_{c}=\frac{2 b}{\sqrt{m^{2}+n^{2}}}$, where $b$ is the length of air void and $m$ and $n$ are mode number.

With these at hand we calculated 3 metallic mesh structures which have $a=2.5 \mu \mathrm{m}, \mathrm{h}$ $=2.0 \mu \mathrm{m}$ and $b=1.0,1.25,1.5 \mu \mathrm{m}$. The reason why we chose $b$ is that when $b=1.0 \mu m$, $\lambda_{s p}>\lambda_{c}, b=1.25 \mu m, \lambda_{s p}=\lambda_{c}$, and $b=1.5 \mu m, \lambda_{s p}<\lambda_{c}$. In Fig. 4.8 (a) - (c) the calculated transmission and reflection are presented and (d) shows the absorption peaks of them.

When surface plasmon wavelength is bigger than waveguide cut-off wavelength, it shows a very sharp absorption peak and small transmission at around $2.5 \mu m$ as shown in Fig. 4.8 (a) and (d). When waveguide cut-off wavelength is bigger than surface plasmon wavelength, the absorption is relatively broad and there is a wide transmission window. Therefore, as an emitter purpose, one better to choose surface plasmon wavelength bigger than waveguide cut-off wavelength. If one wants to use this mesh as a selective filter, one needs to make the structure have waveguide cut-off wavelength bigger than surface plasmon wavelength.

\subsection{Summary and Conclusion}

In summary, we presented the relation between emitting light spectrum and absorption and showed the material and structural dependency of the absorption spectrum. By choosing a proper base material and structural parameters, we can design a selective emitter at a certain region. 


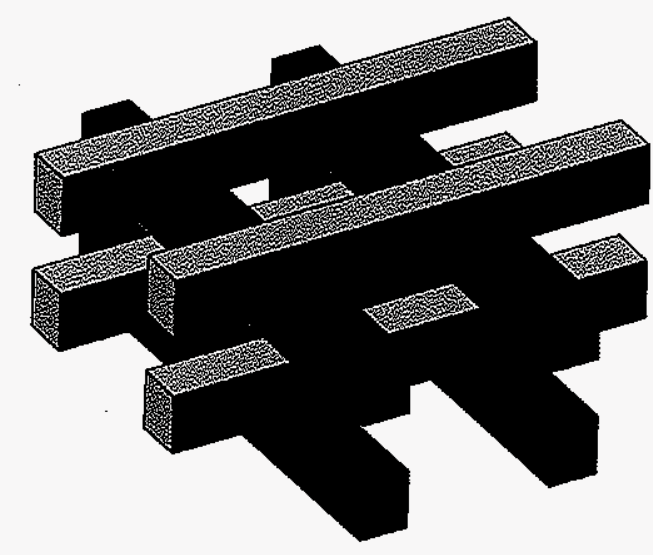

(a) tetragonal

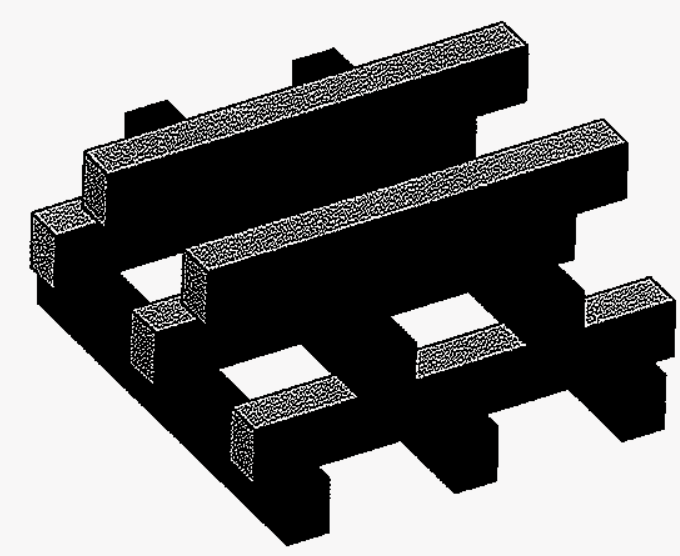

(b) face-centered tetragonal

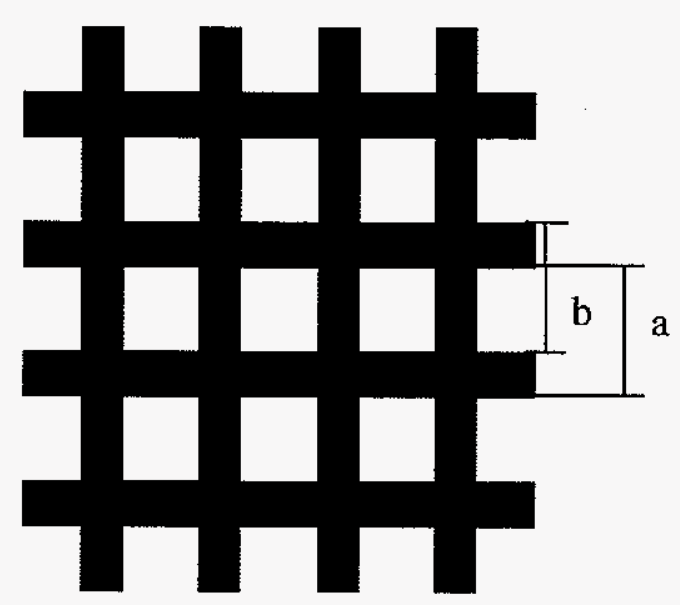

(c)

Fig. 4.1 Structural configurations of woodpile structures and metallic mesh. 


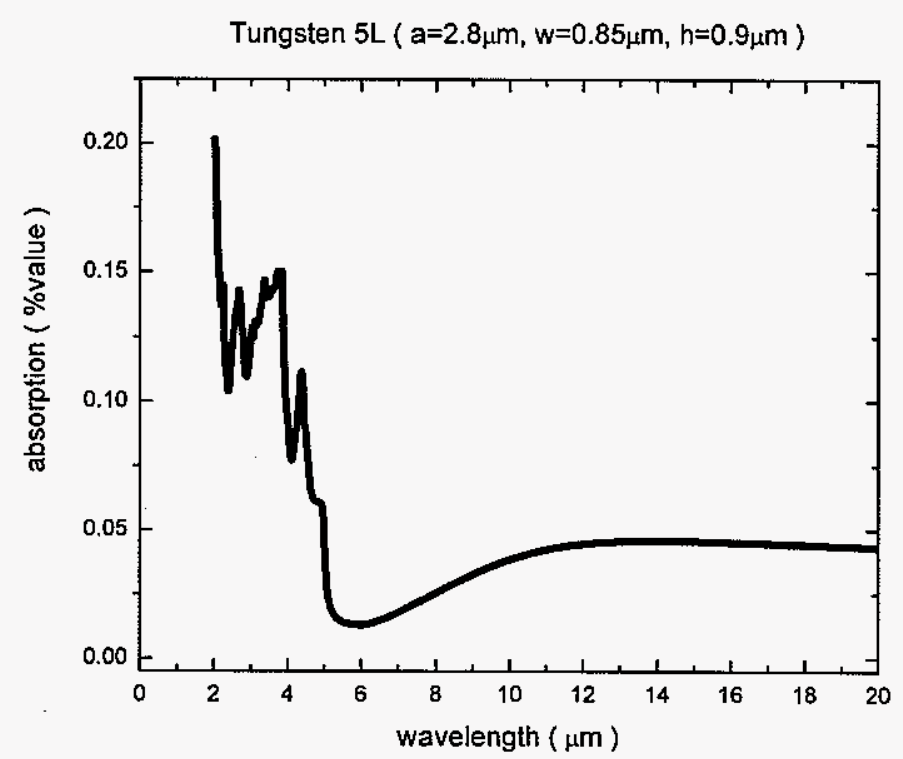

(a)

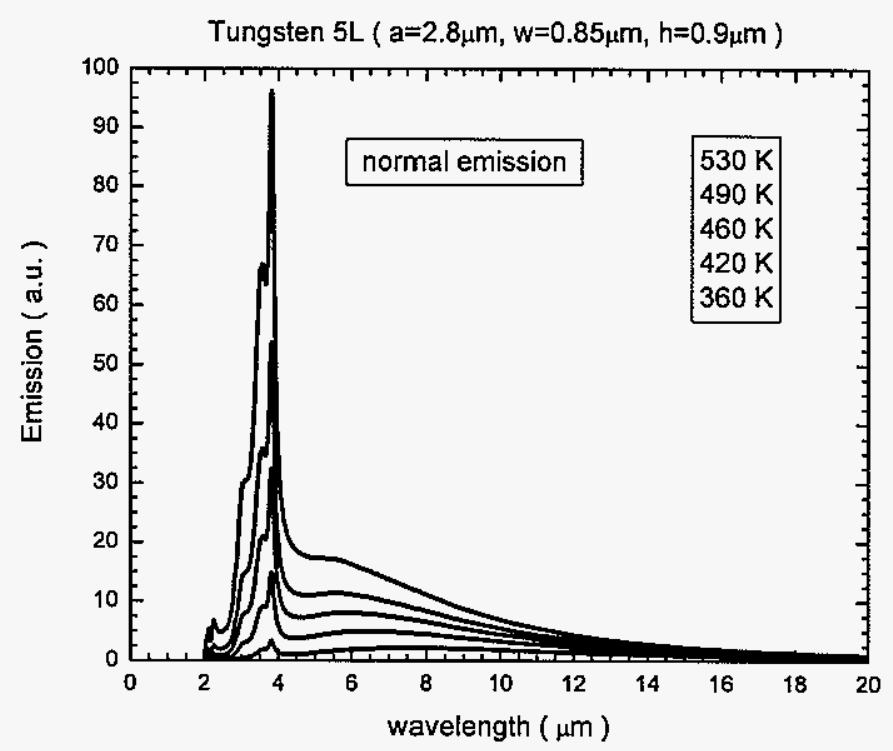

(b)

Fig. 4.2 Simulation results of tungsten 5-layer woodpile structure. (a) is absorption and (b) is emission at various temperature changing. 


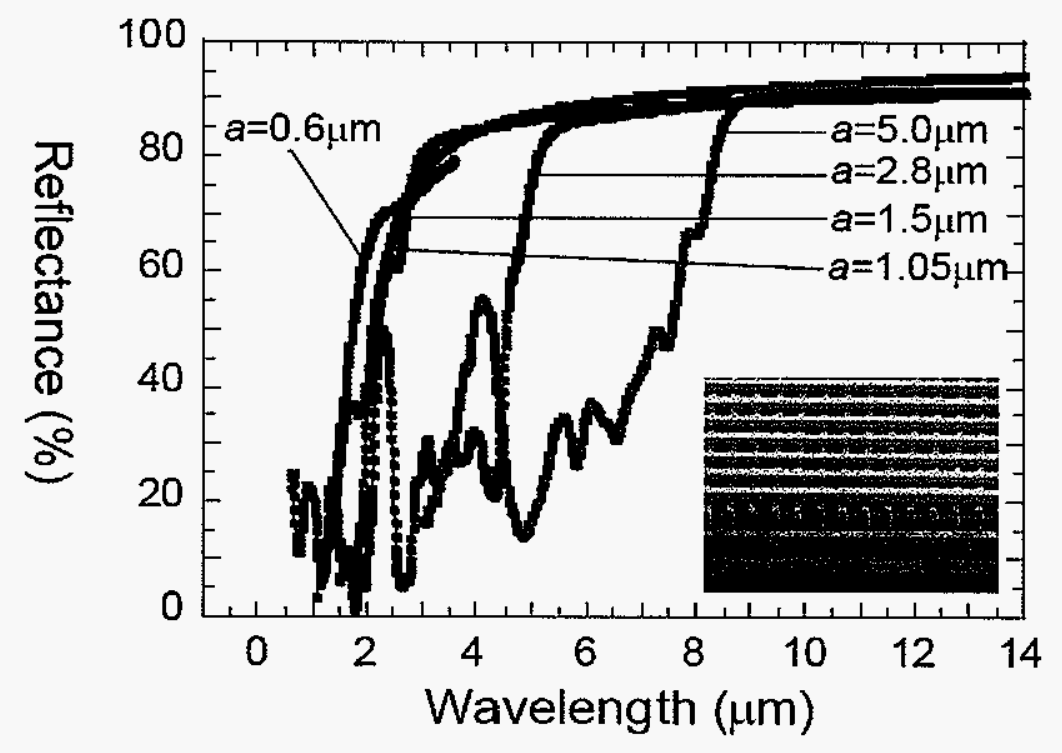

(a)

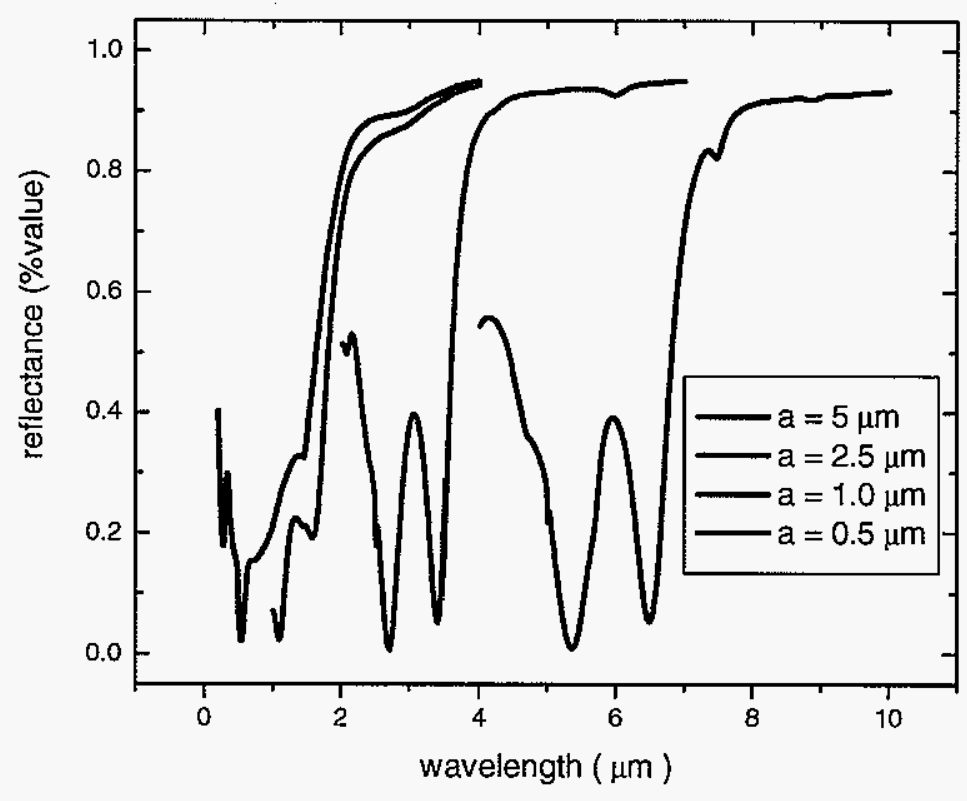

(b)

Fig. 4.3 Band-edge pinning at $\lambda \sim 1.5-2 \mu \mathrm{m}$. (a) is experimental data and (b) is calculated data. 


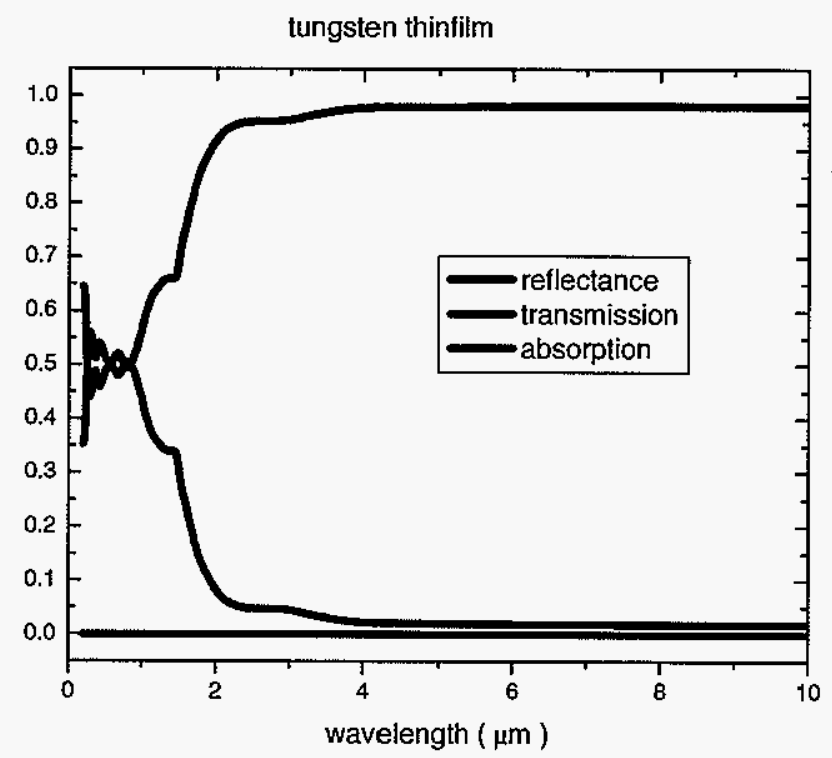

(a)

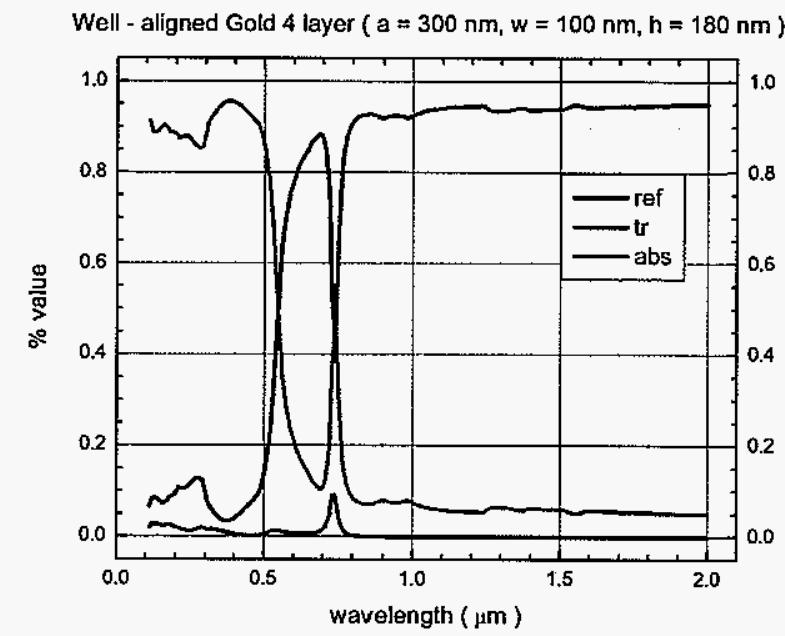

(b)

Fig. 4.4 Calculated optical properties of (a) tungsten thin film ( $500 \mathrm{~nm}$ ) and (b) gold 4-layer photonic crystal. 

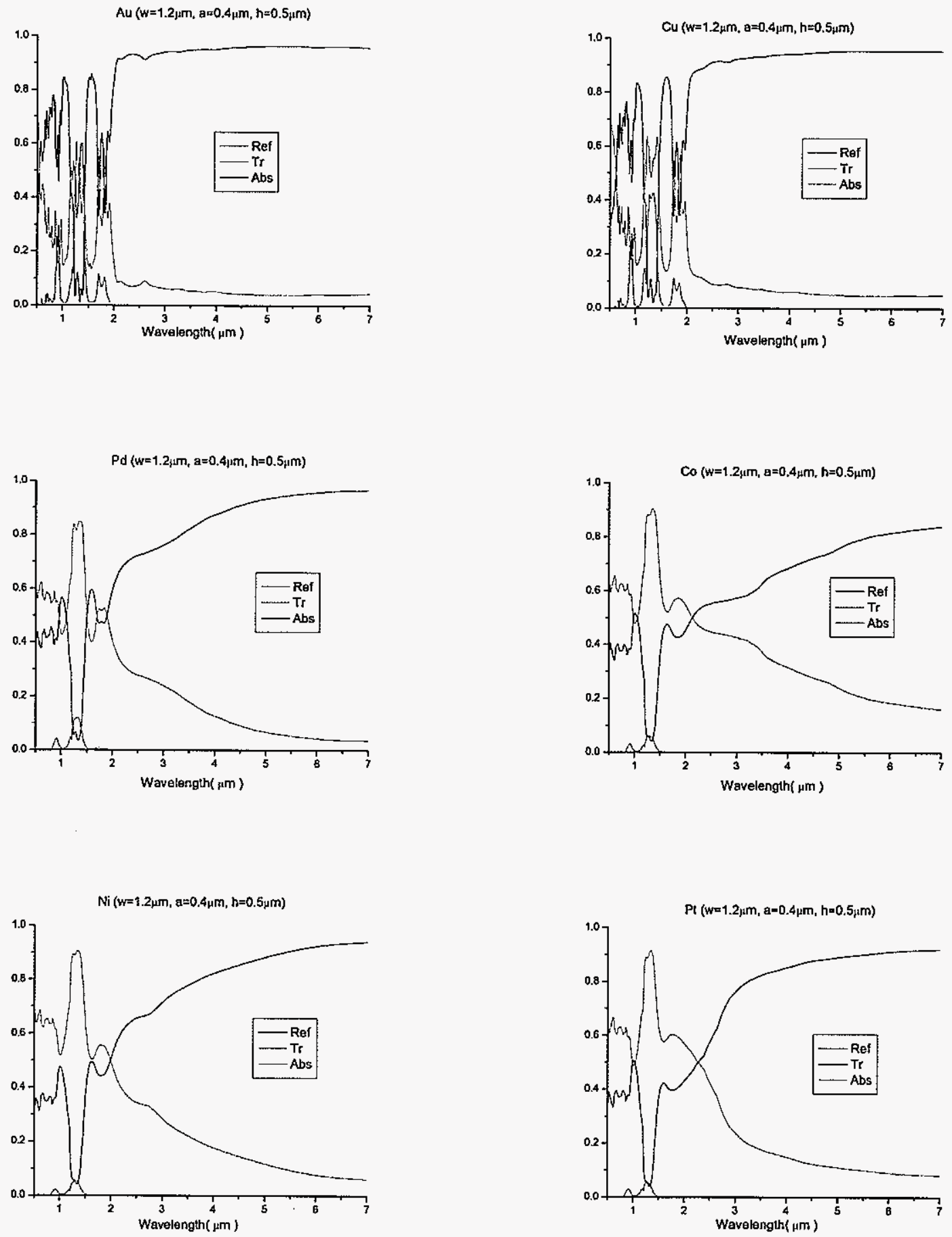

Fig. 4.5 Base materials change MPC's optical properties. 


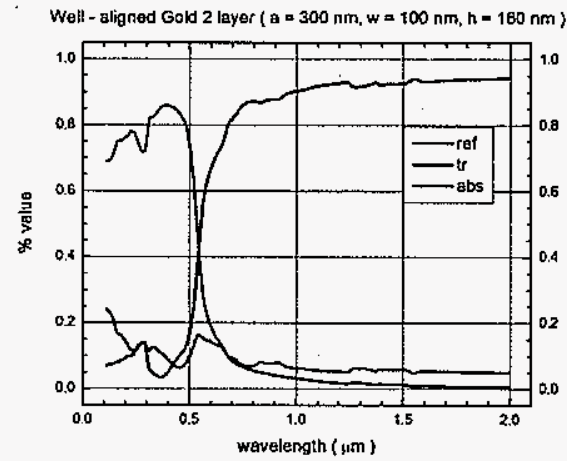

(a)

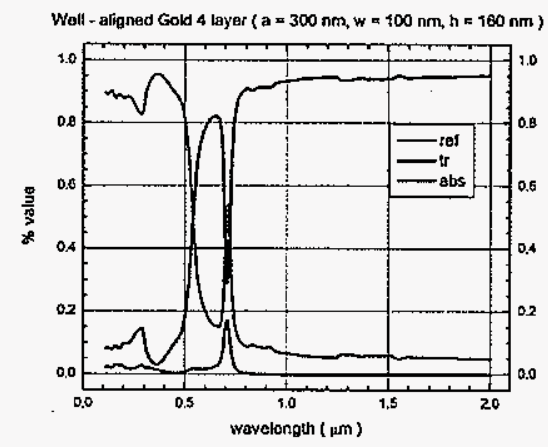

(c)

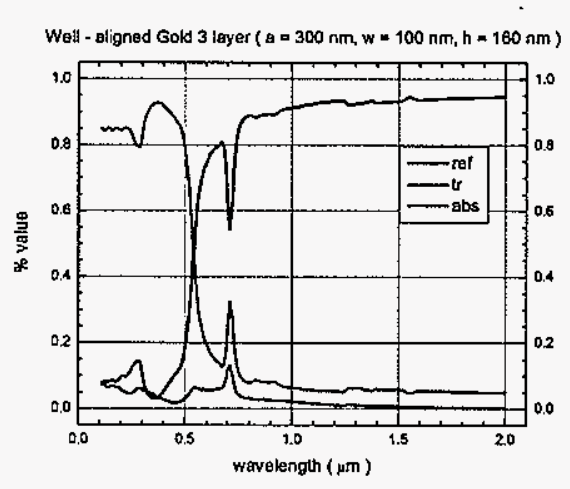

(b)

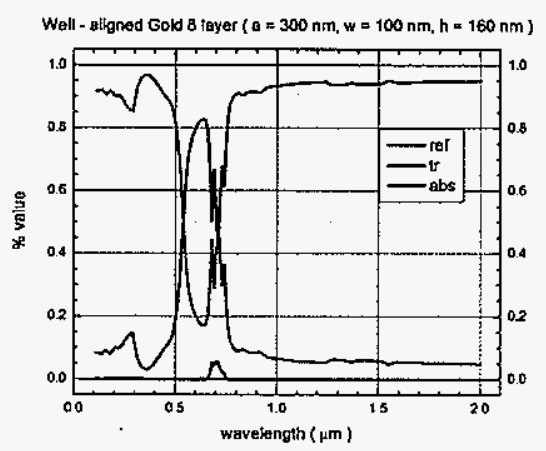

(d)

Fig. 4.6 The number of layers dependency of gold metallic woodpile photonic crystal. 


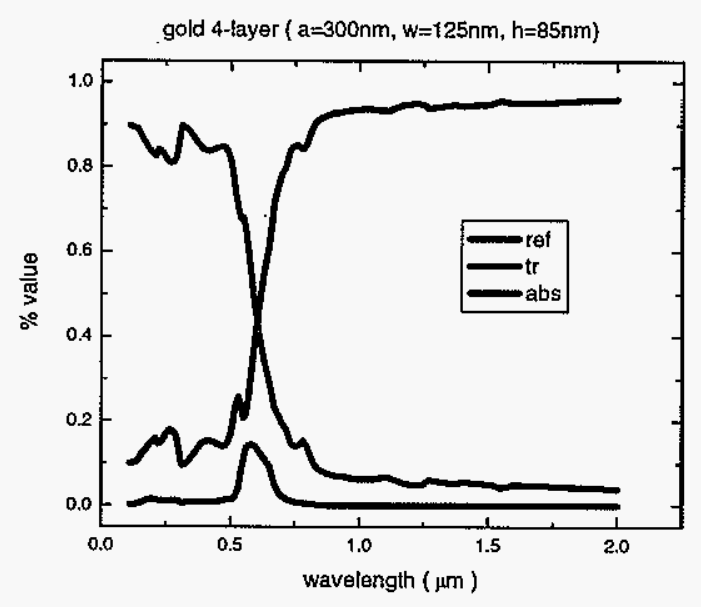

(a)

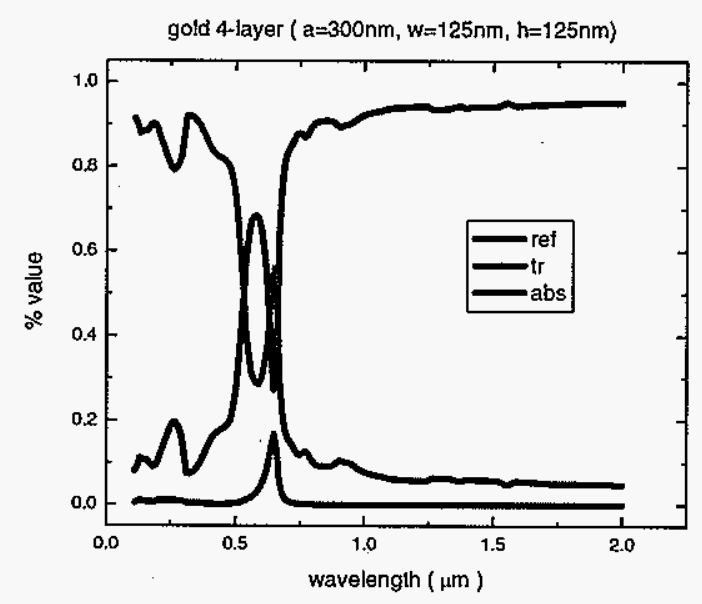

(c)

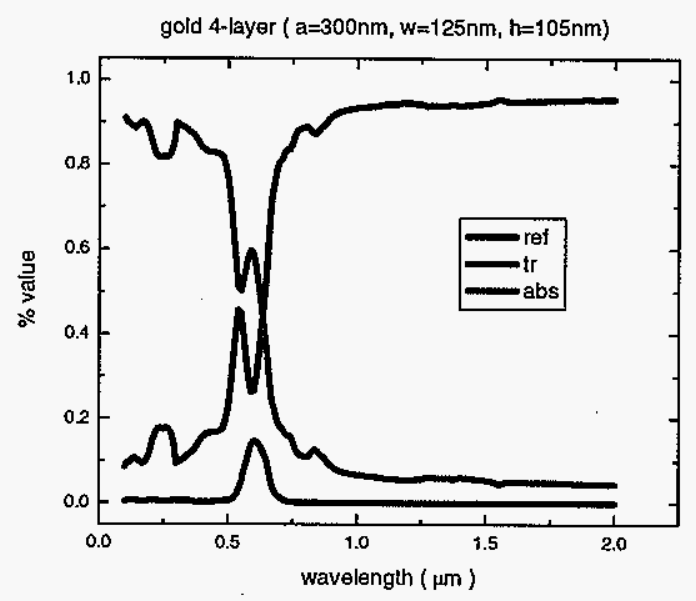

(b)

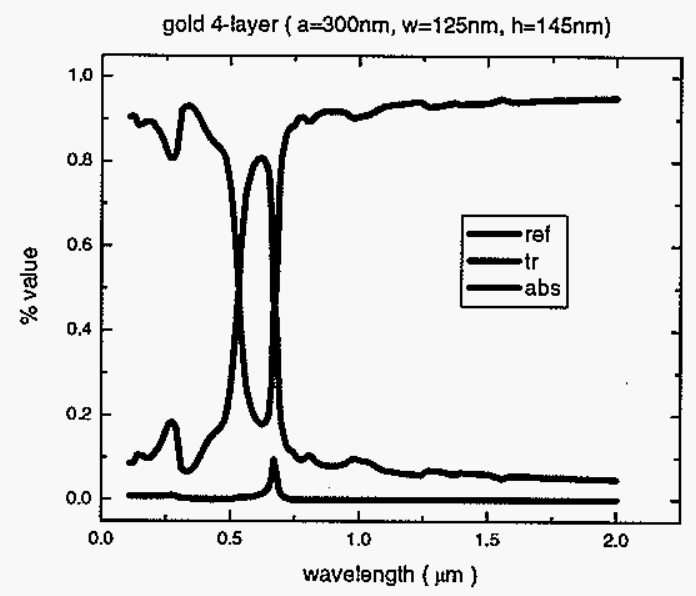

(d)

Fig. 4.7 The bar-height dependency. 


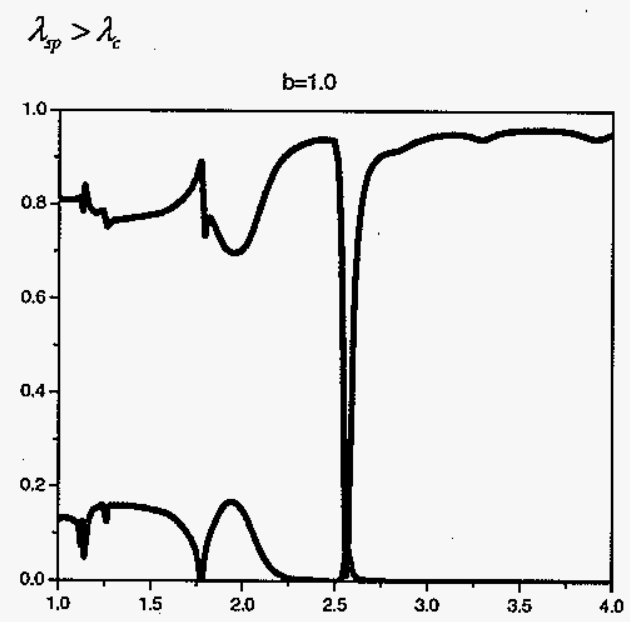

(a)

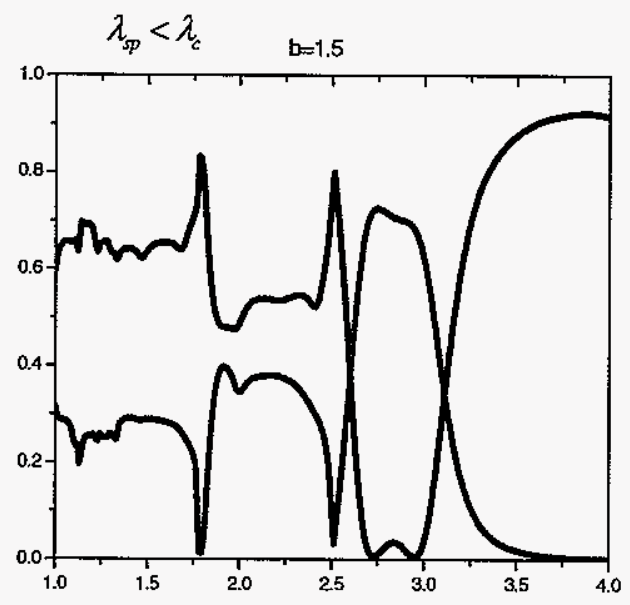

(c)

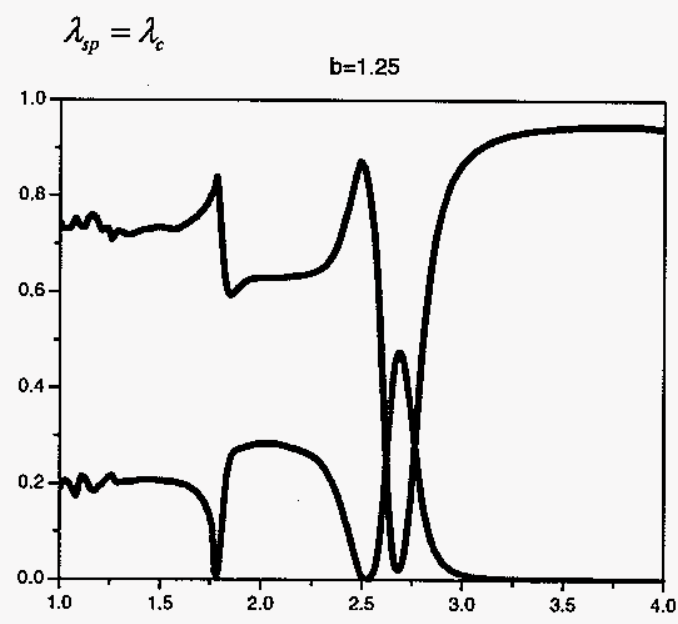

(b)

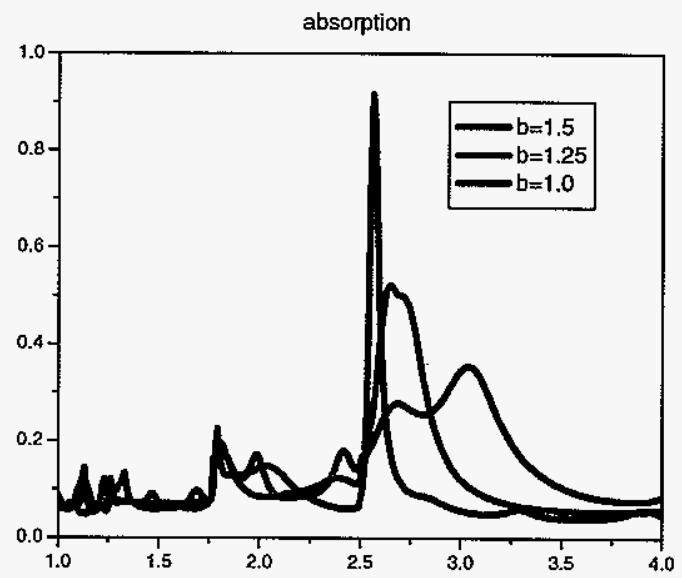

(d)

Fig. 4.8 (a) - (c) Metallic meshes by changing the size of air void where black lines are reflection and red lines are transmission. (d) absorption spectra of metallic meshes. 


\section{CHAPTER 5. Analysis of Bulb System Enclosed by Photonic Structures}

An incandescent light bulb generates light using a glowing filament heated by an electrical current and is widely used in domestic applications, and is the basis of most portable lighting, such as table lamps, some car headlamps and electric flashlights. Incandescent light bulbs, however, are gradually being replaced in many applications, because of their relatively poor energy efficiency. In this chapter we will discuss how we would increase the energy efficiency of an incandescent bulb by using a photonic structure theoretically and show the simulation results.

\subsection{Introduction}

An incandescent light bulb generates light using a glowing filament heated by an electrical current. This light-producing process is known as incandescence and usually contains a vacuum or is filled with a low-pressure noble gas in order to prevent oxidation of the filament at high temperatures. It has been widely used in domestic applications, and has been the basis of most portable lighting, such as table lamps, car headlamps and flashlights. However, because of their relatively poor energy efficiency and short lifetime, incandescent light bulbs are gradually being replaced in many applications by fluorescent lights, highintensity discharge lamps, LEDs, and other devices. In spite of those disadvantages, incandescent lamps are still most familiar light source. The light produced by incandescent 
lamps is a warm tone, emphasizing the yellow/red end of the spectrum. This is one of the reasons incadescent light is still popular. There are some other advantages to incandescent lighting sources, which are easy to dim by using inexpensive controls, easy to direct the light precisely where it is needed. The inefficiency of incandescent lighting sources results from their infrared radiating which is heat rather than visible lights, since their lighting mechanism follow blackbody radition. Therefore, if we can reuse the infrared radiating to increase the temperature of the filament, we can increase the efficiency of the lighting sources. To do this we need to a photonic structure which can return the non-visible lights to the filament.

Following the general ideas outlined above, we arrange this paper as follows. In Sec. 5.2, we will discuss the detail of theories of a blackbody and the role of photonic structures. This will lay down a basis for our later discussions on designing photonic structures. In Sec. 5.3, we will move forward to show designed photonic structures and simulation results of them. Finally in Sec. 5.4, we will present some concluding remarks.

\subsection{Theories of a blackbody and the role of photonic structures}

As we have noticed in the introduction section, the incandescent lighting sources follow blackbody radiation which is encapsulated in the Planck radiation formula, StefanBoltzmann law and Wien's displacement law.

The total radiation power from a blackbody at temperature $T_{b}$ can be divided into two part, visible and non-visible radiation, 


$$
P_{\text {total }}=\sigma T_{b}^{4} A_{b}=P_{\text {vis }}+P_{\text {non-visible }}=A_{b} \int_{\text {visibite }} u\left(\lambda, T_{b}\right) d \lambda+A_{b} \int_{\text {hon-visible }} u\left(\lambda, T_{b}\right) d \lambda
$$

, where $u\left(\lambda, T_{b}\right)$ is radiation energy density from the blackbody filament and $A_{b}$ is the surface area of the blackbody filament.

First, let us calculate the efficiency of a blackbody converting input power to visible light. We calculate blackbody radiation at $2800 \mathrm{~K}$ to see the efficiency which is the ratio of visible radiation power in $360 \mathrm{~nm} \sim 780 \mathrm{~nm}$ to total radiation power, $\int_{360 n m}^{780 n m} \frac{2 \pi h c^{2}}{\lambda^{5}} \frac{1}{e^{h c / k T_{b} \lambda}-1} d \lambda / \sigma T_{b}^{4}$ and we get $\sim 9.8 \%$ as depicted in Fig. 5.1. When it comes to luminous efficiency, the efficiency is $\sim 5.5 \%$ (see Appendix II).

From Eq. (5.1) if we can maintain visible emission ( first term ) high and reduce nonvisible emission (second term) effectively, the efficiency to convert input power to visible light will increase.

To do this, we employed the system which has a blackbody filament enclosed by a photonic structure. Assuming that this system is also enclosed by an ideal enclosure which transmits all the radiation from the system and in which we can keep vacuum as depicted in Fig. 5.2, we can ignore heat conduction, convection and the effect by the enclosure.

When the blackbody radiates its power to the photonic structure, the power relation will be

$$
\begin{aligned}
P_{\text {blackbody }}= & A \int_{\text {all }} u\left(\lambda, T_{b}\right) d \lambda \\
= & P_{\text {input from an extemal source }}+\text { reflected power back to } B B \text { by the photonic structure } \\
& + \text { some portion of radiated power by the photonic structure } \\
\text { Therefore, } &
\end{aligned}
$$


$\begin{aligned} P_{\text {input from an extemat source }}= & A_{b} \int_{\text {all }} u\left(\lambda, T_{b}\right) d \lambda \text {-reflected power back to } B B \text { by the photonic structure } \\ & \text {-some portion of radiated power by the photonic structure }\end{aligned}$

With this at hand, let us check two ideal cases. In the first case, the blackbody filament is enclosed by a photonic structure which transmits all the visible power and reflects all the non-visible power to the blackbody filament, so the supplied power to the blackbody has only two terms. One is the input power from an external source and the other is the reflected radiation from the photonic structure. The third term is zero.

For the case we are looking at, the transmittance and reflectance of the photonic structure, $\operatorname{ref} f_{p s}()=0$ and $t r_{p s}(\lambda)=1$ in visible region. $t r_{p s}(\lambda)=0$ and $r e f \lambda_{p s}()=1$ in nonvisible region, respectively. The reflected power by the photonic structure will be fully absorbed by the blackbody filament, because it is specular reflection (see APPENDIX I) and we don't consider any diffraction by periodic lattices or scattering by surface roughness for this ideal case.

Then, from Eq. (5.2), the power we need to input is

$$
P_{\text {input }}=A_{b} \int_{\text {all }} u\left(\lambda, T_{b}\right) d \lambda-A_{b} \int_{\text {all }} r e f_{p s}(\lambda) u\left(\lambda, T_{b}\right) d \lambda
$$

For this case, Eq. (5.3) becomes

$$
P_{\text {input }}=A_{b} \int_{\text {all }} u\left(\lambda, T_{b}\right) d \lambda-A_{b} \int_{\text {non-visible }} u\left(\lambda, T_{b}\right) d \lambda=A_{b} \int_{\text {visible }} u\left(\lambda, T_{b}\right) d \lambda \text { and this is reasonable, }
$$

because we need to supply external input power only by the amount of transmitted power to keep the temperature of the filament a constant. 
In the second case the blackbody filament is enclosed by a photonic structure which transmits all the visible power and absorbs all the non-visible power. In this case the second term of Eq.(5.2) is zero and the third term is not zero. The photonic structure will radiate and only some portion of it will be absorbed by the blackbody filament. The portion of the energy back to the blackbody is $\left(1-\frac{\sqrt{r_{p s}^{2}-r_{b}^{2}}}{r_{s p}}\right)$ (see APPENDIX I).

where $r_{b}$ is the radius of the blackbody and $r_{p s}$ is the radius of the photonic structure.

The power we need to input is, then,

$$
P_{\text {input }}=A_{b} \int_{0}^{\infty} u\left(\lambda, T_{b}\right) d \lambda-\left(1-\frac{\sqrt{r_{p s}^{2}-r_{b}^{2}}}{r_{s p}}\right)\left(A_{p s}\right) \int_{0}^{\infty} a b s_{p s}(\lambda) u\left(\lambda, T_{p s}\right) d \lambda
$$

To solve Eq. (5.4) we need to relate $\int_{0}^{\infty} a b s_{p s}(\lambda) u\left(\lambda, T_{p s}\right) d \lambda$ to $\int_{0}^{\infty} u\left(\lambda, T_{b}\right) d \lambda$. To do that, let us think about the power absorbed and emitted by the photonic structure. The absorbed power by the photonic structure from the blackbody filament and the photonic structure itself is

$$
A_{b} \times \int_{0}^{\infty} a b s_{p s}(\lambda) u\left(\lambda, T_{b}\right) d \lambda+A_{p s} \times \frac{\sqrt{r_{p s}^{2}-r_{b}^{2}}}{r_{p s}} \int_{0}^{\infty} a b s_{p s}(\lambda) u\left(\lambda, T_{p s}\right) d \lambda
$$

The emitted power by the photonic structure is

$$
2 \times A_{p s} \times \int_{0}^{\infty} a b s_{p s}(\lambda) u\left(\lambda, T_{p s}\right) d \lambda
$$


where the factor 2 is multiplied because the photonic structure has two sides.

In equilibrium, Eq. (5.5) and Eq. (5.6) should be the same, therefore, we have

$$
\begin{aligned}
& A_{b} \times \int_{0}^{\infty} a b s_{p s}(\lambda) u\left(\lambda, T_{b}\right) d \lambda+A_{p s} \times \frac{\sqrt{r_{p s}^{2}-r_{b}^{2}}}{r_{p s}} \int_{0}^{\infty} a b s_{p s}(\lambda) u\left(\lambda, T_{p s}\right) d \lambda \\
& =2 \times A_{p s} \times \int_{0}^{\infty} a b s_{p s}(\lambda) u\left(\lambda, T_{p s}\right) d \lambda
\end{aligned}
$$

and

$$
A_{b} \times \int_{0}^{\infty} a b s_{p s}(\lambda) u\left(\lambda, T_{b}\right) d \lambda=A_{p s} \times\left(2-\frac{\sqrt{r_{p s}^{2}-r_{b}^{2}}}{r_{p s}}\right) \int_{0}^{\infty} a b s_{p s}(\lambda) u\left(\lambda, T_{p s}\right) d \lambda
$$

Therefore,

$$
A_{p s} \times \int_{0}^{\infty} a b s_{p s}(\lambda) u\left(\lambda, T_{p s}\right) d \lambda=A_{b} \times\left(2-\frac{\sqrt{r_{p s}^{2}-r_{b}^{2}}}{r_{p s}}\right)^{-1} \int_{0}^{\infty} a b s_{p s}(\lambda) u\left(\lambda, T_{b}\right) d \lambda
$$

Plug Eq. (5.7) into Eq. (5.4) and we get

$$
P_{\text {input }}=A_{b} \times \int_{0}^{\infty} u\left(\lambda, T_{b}\right) d \lambda-\left(\frac{r_{p s}-\sqrt{r_{p s}^{2}-r_{b}^{2}}}{2 r_{p s}-\sqrt{r_{p s}^{2}-r_{b}^{2}}}\right) A_{b} \times \int_{0}^{\infty} a b s_{p s}(\lambda) u\left(\lambda, T_{b}\right) d \lambda
$$

For the case we are looking at, $a b s_{p s}(\lambda)=0$ in visible region and $a b s_{p s}(\lambda)=1$ in non-visible region. Therefore, Eq. (5.8) becomes

$$
P_{\text {input }}=A_{b} \times\left[\int_{\nu i s i b l e} u\left(\lambda, T_{b}\right) d \lambda+\left(1-\frac{r_{p s}-\sqrt{r_{p s}^{2}-r_{b}^{2}}}{2 r_{p s}-\sqrt{r_{p s}^{2}-r_{b}^{2}}}\right) \int_{\text {hon-visible }} u\left(\lambda, T_{b}\right) d \lambda\right]
$$




$$
=A_{b} \times\left[\int_{\text {ivisible }} u\left(\lambda, T_{b}\right) d \lambda+\left(\frac{r_{p s}}{2 r_{p s}-\sqrt{r_{p s}^{2}-r_{b}^{2}}}\right) \int_{\text {non-visible }} u\left(\lambda, T_{b}\right) d \lambda\right]
$$

To check the temperature of the photonic structure we need to solve Eq. (5.7) about $T_{p s .}$. If $a b s_{p s}(\lambda)$ is not a constant, we have to know exact dimensions of the system, the temperature of the blackbody and $a b s_{p s}(\lambda)$ and then we can calculate the temperature of the photonic structure numerically. If $a b s_{p s}(\lambda)$ is a constant, i.e. the photonic structure is a blackbody or greybody, Eq. (5.7) becomes

$$
\begin{aligned}
A_{b} \int_{0}^{\infty} u\left(\lambda, T_{b}\right) d \lambda & =A_{p s}\left(2-\frac{\sqrt{r_{p s}^{2}-r_{b}^{2}}}{r_{p s}}\right)_{0}^{\infty} u\left(\lambda, T_{p s}\right) d \lambda \\
A_{b} \sigma T_{b}^{4} & =A_{p s}\left(2-\frac{\sqrt{r_{p s}^{2}-r_{b}^{2}}}{r_{p s}}\right) \times \sigma T_{p s}^{4} \\
T_{p s} & =\sqrt{\frac{A_{b}}{A_{p s}\left(2-\frac{\sqrt{r_{p s}^{2}-r_{b}^{2}}}{r_{p s}}\right)}} T_{b}
\end{aligned}
$$

and if they are spheres, then

$$
T_{p s}=\sqrt[4]{\frac{r_{b}^{2}}{r_{p s}^{2} \times\left(2-\frac{\sqrt{r_{p s}^{2}-r_{b}^{2}}}{r_{p s}}\right)}} T_{b}
$$


For the realistic case, the temperature of the photonic structure should be lower than its melting temperature of the material building the photonic structure to avoid burning out.

Therefore,

$$
T_{m t}>T_{p s}=\sqrt[4]{\frac{r_{b}^{2}}{r_{p s}^{2} \times\left(2-\frac{\sqrt{r_{p s}^{2}-r_{b}^{2}}}{r_{p s}}\right)^{2}}} T_{b}
$$

where $T_{m t}$ is the melting temperature of the photonic structure.

Now, we apply these results to more general case in which the photonic structure has continuously changing reflectance and absorption by wavelengths. From Eq. (5.3) and Eq. (5.8), we can get a power relation for the system which consists of a blackbody filament and a photonic structure,

$$
P_{\text {input }}=A_{b} \int_{0}^{\infty}\left[1-r e f_{p s}(\lambda)-\left(\frac{r_{p s}-\sqrt{r_{p s}^{2}-r_{b}^{2}}}{2 r_{p s}-\sqrt{r_{p s}^{2}-r_{b}^{2}}}\right) a b s_{p s}(\lambda)\right] u\left(\lambda, T_{b}\right) d \lambda
$$

Now, let us define the converting efficiency which is the ratio of output visible light power to external input power

$$
\text { eff }=\frac{P_{\text {visible }}}{P_{\text {input }}}=\frac{A_{b} \int_{\text {visible }} t r_{p s}(\lambda) u\left(\lambda, T_{b}\right) d \lambda+A_{p s} \int_{v i s i b l e} a b s_{p s}(\lambda) u\left(\lambda, T_{p s}\right) d \lambda}{A_{b} \int_{0}^{\infty}\left[1-r e f_{p s}(\lambda)-\left(\frac{r_{p s}-\sqrt{r_{p s}^{2}-r_{b}^{2}}}{2 r_{p s}-\sqrt{r_{p s}^{2}-r_{b}^{2}}}\right) a b s_{p s}(\lambda)\right] u\left(\lambda, T_{b}\right) d \lambda}
$$

and the resulting power spectra is 


$$
\left[A_{b} t r_{p s}(\lambda) u\left(\lambda, T_{b}\right)+A_{p s} a b s_{p s}(\lambda) u\left(\lambda, T_{p s}\right)\right] d \lambda
$$

Until now, we examined the case in which there's no diffraction of reflected lights. However, for the practical purpose, we need to think about the case of diffractions, because the photonic structure we will use in the next section would make diffraction due to its geometry.

The upper limit of the wavelength that will be diffracted by a grating is

$$
\lambda=\frac{a}{m}(\sin \alpha+\sin \beta)
$$

,where $\alpha$ is an incident angle, $\beta$ is a diffracted angle and $a$ is the periodicity of a photonic structure. The photonic structures we will examine have pitches smaller than $500 \mathrm{~nm}$ and the upper limit of $1^{\text {st }}$ order diffraction is $\lambda=(500 \mathrm{~nm})(\sin \alpha+\sin \beta)$. The incident angle $\alpha$ is defined as $\sin \alpha=\frac{r_{b}}{r_{p s}}$ and it is $\sim 0.083$ in our specific design $\left(r_{b}: r_{p s}=1: 12\right)$, then $\lambda_{\max } \cong 542 \mathrm{~nm}$. This is in visible range we want to make it transmit through the photonic structure.

Now, let us calculate the approximate power of diffracted lights. The power from the blackbody in $0 \mathrm{~nm} \sim 542 \mathrm{~nm}$ is below $2 \%$ of its total energy and almost $90 \%$ of this will be transmitted with our specific photonic structures and $\sim 5 \%$ will be reflected, so below $0.1 \%$ of the total power will be reflected with diffractions. We compared the intensities of $0^{\text {th }}$ and $1^{\text {st }}$ order diffractions and get the intensity of $1^{\text {st }}$ order is $\sim 19 \%$ of $0^{\text {th }}$ order when the filling 
fraction is $\sim 35 \%$. If the filling fraction is smaller, the intensity is also getting smaller. The overall intensity of higher diffractions is $\sim 0.02 \%$ of the total power of the blackbody and it might be negligible. Therefore, we considered only $0^{\text {th }}$ order reflection, which is specular, for the rest of calculations.

\subsection{Results and Discussion}

In the previous section we have developed theoretical tool which enables us to solve efficiently the power relation between a blackbody filament and a photonic structure. With this at hand let us proceed to design photonic structures which are suitable to increase the efficiency of visible lights radiation.

First, we present the efficiency of two ideal cases. Fig.5.3 (a) shows the transmittance and absorption of an ideal photonic structure which has $100 \%$ transmittance in visible-light region and $100 \%$ absorption in non-visible light region. In this case $100 \%$ of lights in visible region transmits the photonic structure and radiated lights from the photonic structure in nonvisible region are emitted to the outside of it as shown in Fig.5.3 (c). The red line shows the emitted lights from the blackbody filament at $2800 \mathrm{~K}$ and the blue line shows the lights after

filtering with the photonic structure In Fig.5.3 (c). The efficiency of this case can be calculated with Eq. (5.3). If the temperature of the filament is $2800 \mathrm{~K}$, the efficiency of the system is about $9.83 \%$ as shown below and it is almost the same as that of a single blackbody system. 
In Fig.5.3 (b) and (d), we show the result of an ideal photonic structure which has $100 \%$ transmittance in visible-light region and $100 \%$ reflectance in non-visible light region. In this case $100 \%$ of lights in visible region transmits the photonic structure and $0 \%$ of lights in non-visible region is emitted to the outside of it. The efficiency of this case is $100 \%$.

These results clearly show that we need to design a photonic structure to have high transmittance in visible region and high reflectance in non-visible region.

We chose silver as a base material which has low intrinsic absorption in visible and infrared region. It is necessary that the base material should have low intrinsic absorption in the region, because we need high transmittance in visible region and high reflectance in nonvisible region. The high absorption of silver in ultraviolet region does not affect much (but ; non-zero) because blackbody radiation in the region is very small at $\sim 2800 \mathrm{~K}$.

The geometric configuration of the photonic structure we will investigate is schematically illustrated in Fig. 4. It is metallic mesh arranged in a square lattice. We also assumed that the photonic structure is far enough from the filament to make its temperature smaller than its melting temperature $\left(r_{b}: r_{p s}=1: 12\right)$. We used published $(n, k)$ values $[44]$ to calculate these optical values.

We fixed the size of air void $380 \mathrm{~nm}$ because it is a half of waveguide cut-off wavelength, $780 \mathrm{~nm}$, which is the maximum wavelength in visible region. We calculated the efficiency of converting input energy to visible light by filling fraction. The filling fraction is defined as w/d in Fig.5.4.

In Fig.5.5 we present the efficiency by filling fraction. When filling fraction is $25 \%$, the maximum efficiency, $42.96 \%$, is achieved. To have these results, we first calculated the 
reflectance, transmittance and absorption of the metallic mesh structures by using Transfer Matrix Method[7,8] and we fixed the thickness of the structure at $500 \mathrm{~nm}$ and the size of airvoid at $380 \mathrm{~nm}$. Then, the efficiencies are calculated by using Eq. (5.13).

In Fig.5.6 we also present the thickness-dependency of efficiency. We chose the filling fraction of $20 \%$ and $25 \%$ and varied the thickness from $300 \mathrm{~nm}$ to $900 \mathrm{~nm}$. The efficiencies show the maxima at around $500 \mathrm{~nm}$ thick at which the photonic structure is thick enough to attenuate the transmission of longer wavelengths. The maximum efficiencies are $\sim 41.53 \%$ for the filling fraction of $20 \%$ and $\sim 42.96 \%$ for the filling fraction of $25 \%$, respectively, at the thickness of $500 \mathrm{~nm}$. Comparing with the efficiency of $9.8 \%$ for a bare blackbody they have more than four times bigger efficiencies.

The resulting spectrum of the filling fraction of $25 \%$ and the thickness of $500 \mathrm{~nm}$ is shown in Fig.5.7. We can clearly see that the resulting spectrum shows almost a blackbody radiation in visible region and strong attenuation in infrared region.

\subsection{Summary and Conclusion}

In summary, we have developed a theoretical model to analyze a blackbody enclosed by a metallic mesh which can increase the efficiency of converting a blackbody radiation to visible light. With this model we found that a square lattice metallic mesh enclosing a filament might increase the efficiency of incandescent lighting sources. Filling fraction and thickness dependency were examined and presented. Combining these two parameters is essential to achieve the maximum output result. 


\section{Appendix I. Reflected or Absorbed Beam by a Photonic Structure}

\section{A.1.1 Ideal Reflection}

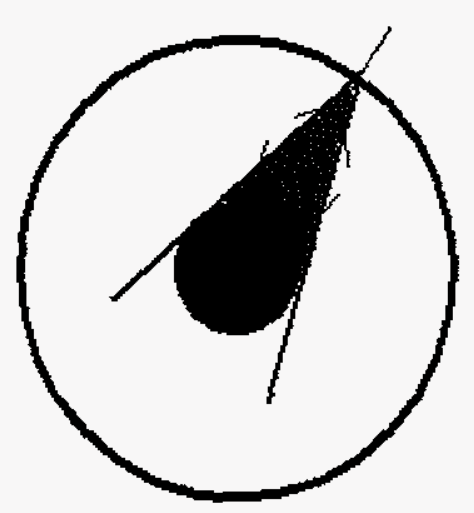

Fig. A1.1 All the reflected beam by a photonic structure go back to a blackbody.

Let us first discuss the case in which $\operatorname{tr}\left(\lambda_{1}\right)=0, a b s\left(\lambda_{1}\right)=0$ and $\operatorname{ref}\left(\lambda_{1}\right)=1$ at $\lambda=\lambda_{1}$. In this case there's no absorption by the photonic structure but reflection. A spot on a blackbody radiates to all the angles and the radiated beam from the blackbody will be specularly reflected by a photonic structure. We are assuming that there is no diffraction by periodic lattices of photonic structure nor scattering by surface roughness of the photonic structure until the photonic structure is specified.

As shown in Fig. A1.1, if we take a spot on the photonic structure, the radiated beam from the blackbody toward the spot in the red region can only reach the spot on the photonic 
structure and will be reflected specularly, so all of them will be absorbed by the blackbody again.

\section{A.1.2 Ideal Absorption}

Next, let us discuss the case in which $\operatorname{tr}\left(\lambda_{l}\right)=0, a b s\left(\lambda_{l}\right)=1$ and $\operatorname{ref}\left(\lambda_{l}\right)=0$ at $\lambda=$ $\lambda_{1}$. For this case there's no reflection but absorption. As shown in Fig. A1.2, when the radiation from a spot on the photonic structure falls into the cone of the angle $2 \alpha$, it will be absorbed by the blackbody, otherwise it will be absorbed by the photonic structure.

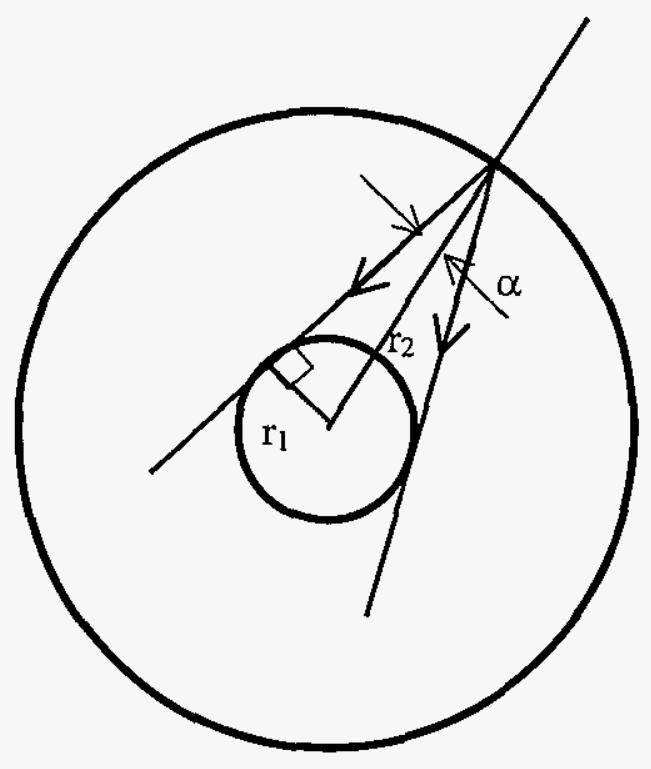

Fig. A1.2. Configuration of absorption. 
The solid angle of the cone is $2 \pi(1-\cos \alpha)$ and we have $2 \pi\left(1-\frac{\sqrt{r_{2}^{2}-r_{1}^{2}}}{r_{2}}\right)$. $2 \pi\left(1-\frac{\sqrt{r_{2}^{2}-r_{1}^{2}}}{r_{2}}\right) / 2 \pi=\left(1-\frac{\sqrt{r_{2}^{2}-r_{1}^{2}}}{r_{2}}\right)$. This portion of radiated power from the photonic structure will go back to the blackbody. The portion back to the photonic structure is $\frac{\sqrt{r_{2}^{2}-r_{1}^{2}}}{r_{2}}$ 


\section{Appendix II. Luminous Efficiency}

A light can waste power by emitting too much light outside of the visible spectrum. Only visible light is useful for illumination, and some wavelengths are perceived as brighter than others. Taking this into account, luminous efficacy is a ratio of the useful power emitted to the total power and is measured in lumens per watt $(\mathrm{lm} / \mathrm{W})$. The maximum efficacy possible is $683 \mathrm{~lm} / \mathrm{W}$. Luminous efficiency is a function of luminous efficacy divided by this maximum and thus is expressed as a number between 0 and 1 , or as a percentage. The procedure of calculation is described below.

$$
\begin{aligned}
& \text { lumens }=\sum_{\lambda} 683 u\left(\lambda, T_{b}\right) \times L \cdot E .(\lambda) \\
& \text { total luminous efficiency }=\left(\frac{\text { lumens }}{683 \text { lumens } / \text { watt }}\right) /(\text { input power })
\end{aligned}
$$

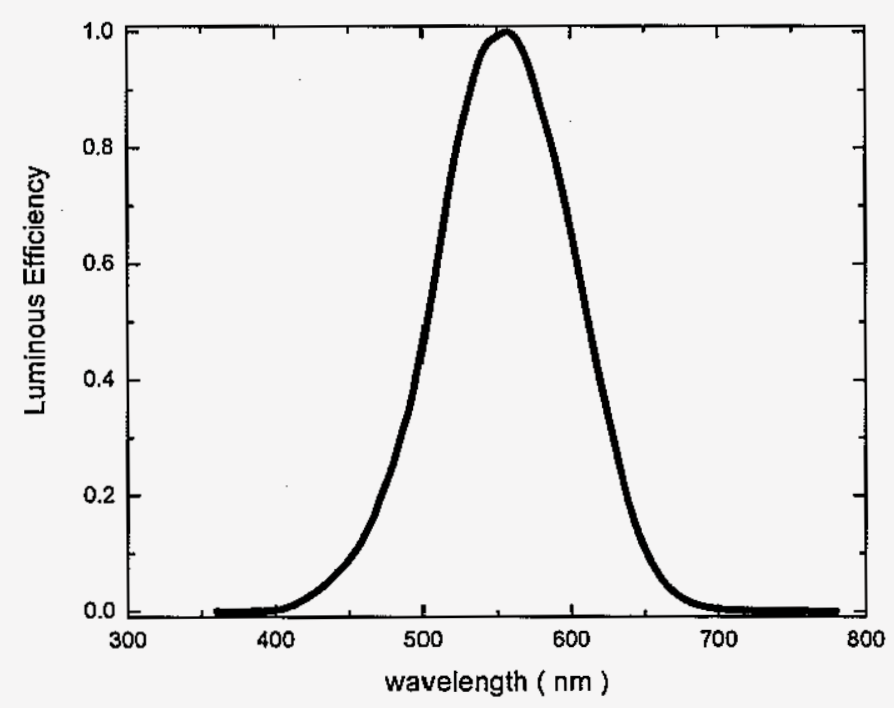

Fig. A2.1 Luminous efficient curve. 


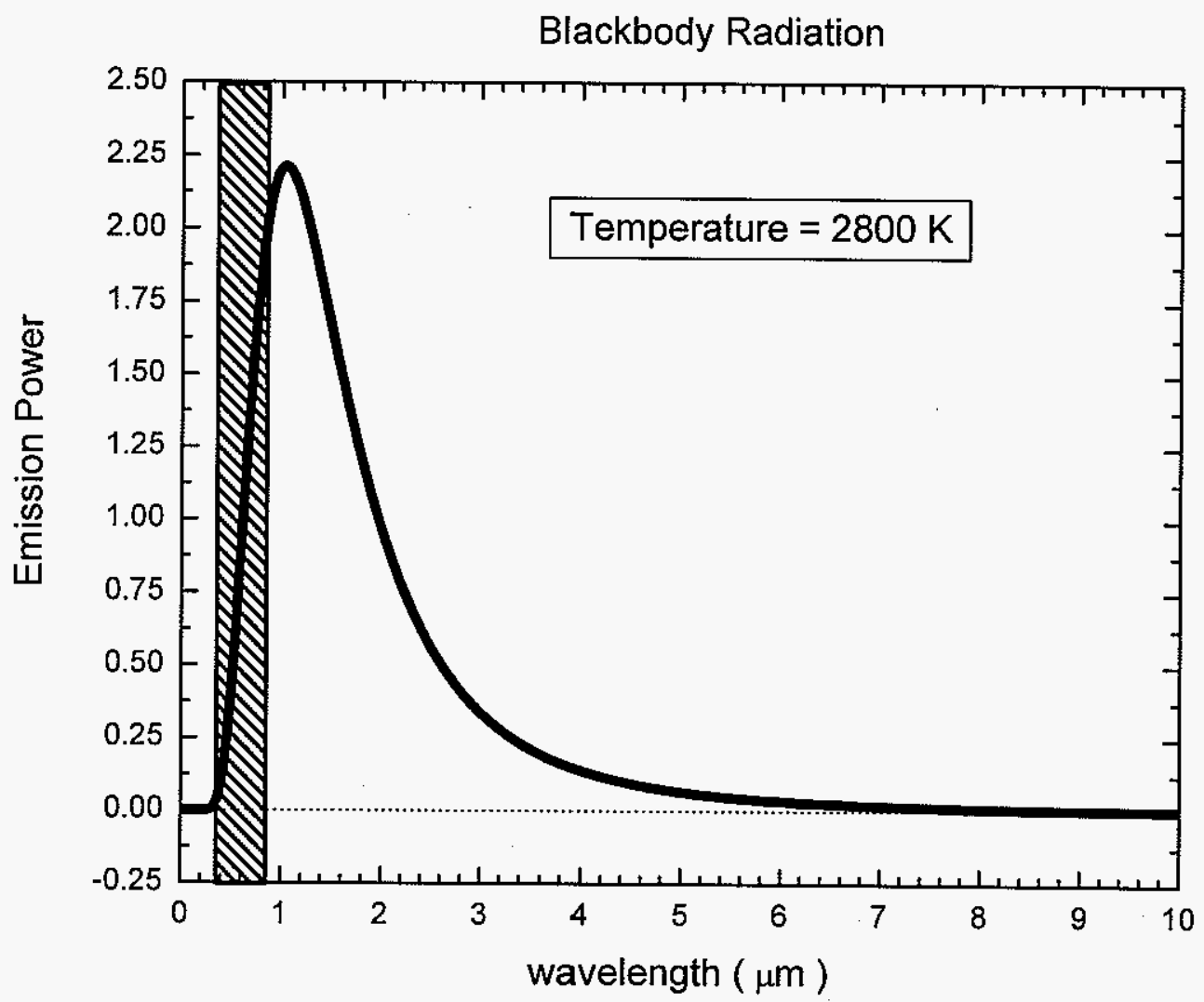

Fig. 5.1 A blackbody radiation at $2800 \mathrm{~K}$. 


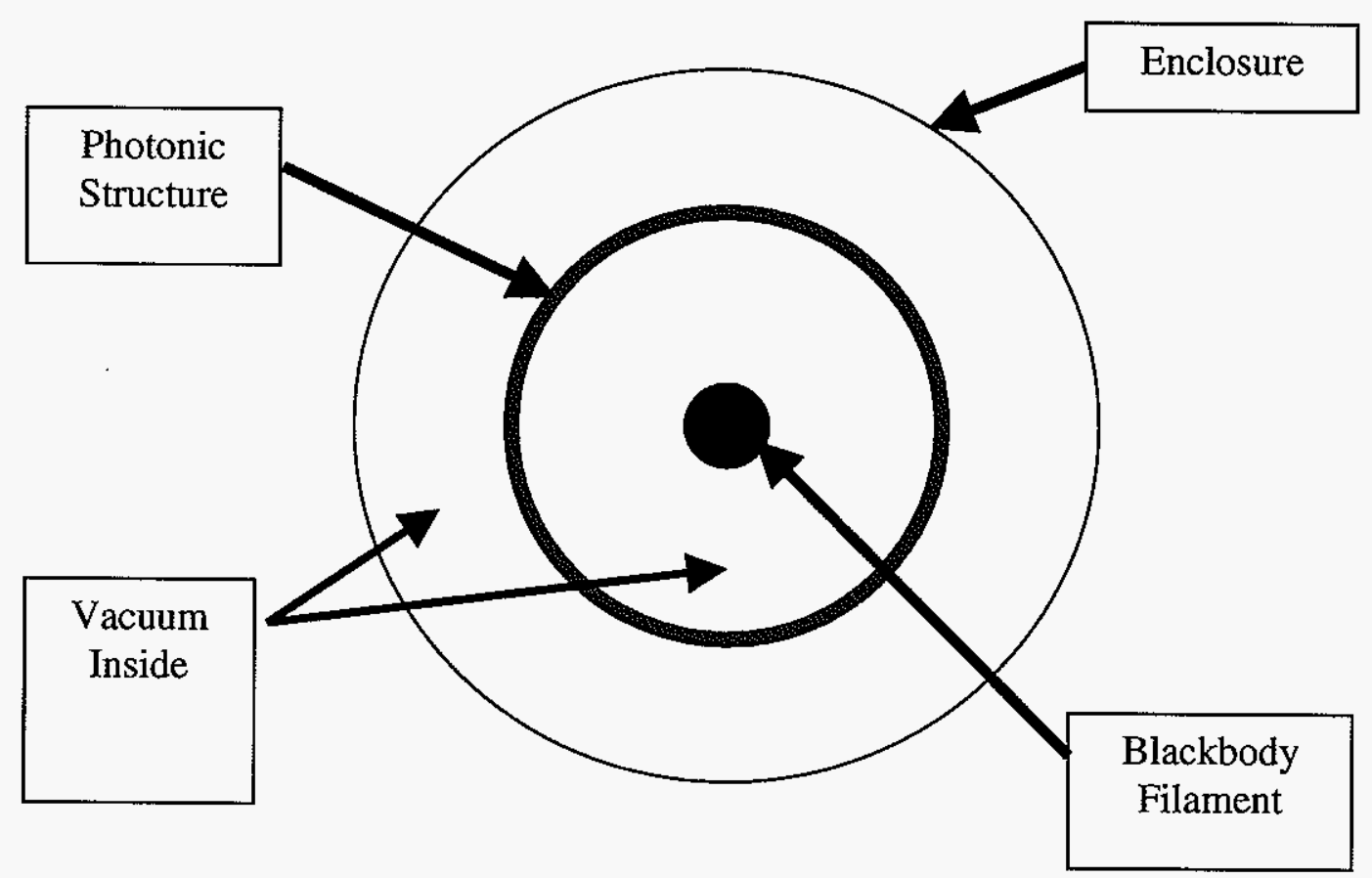

Fig. 5.2 Schematic representation of a blackbody filament enclosed by a photonic structure. 


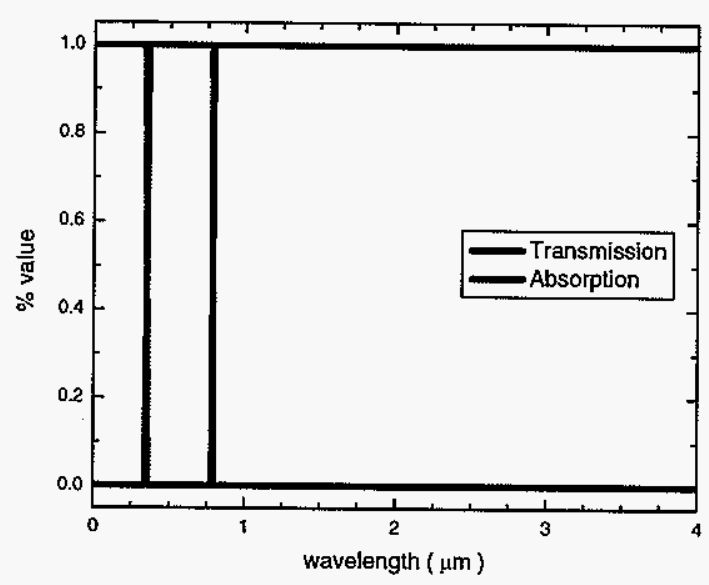

(a)

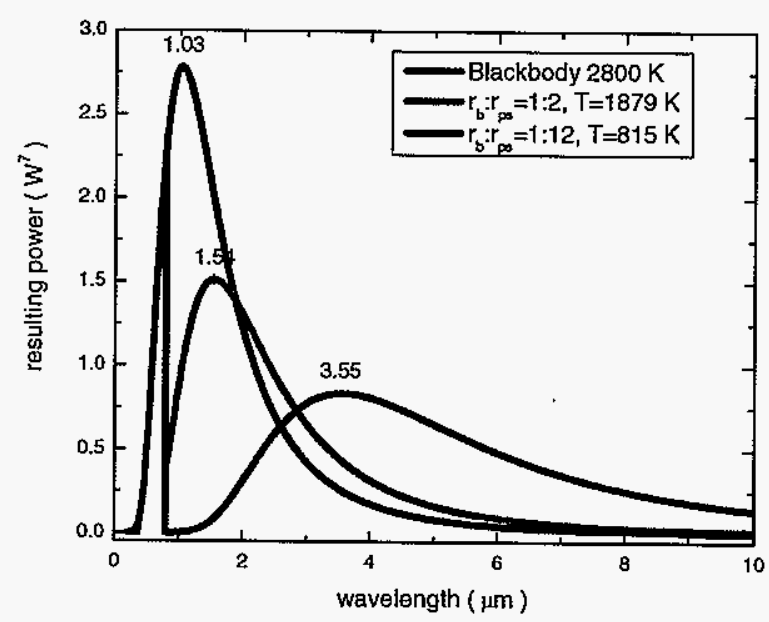

(c)

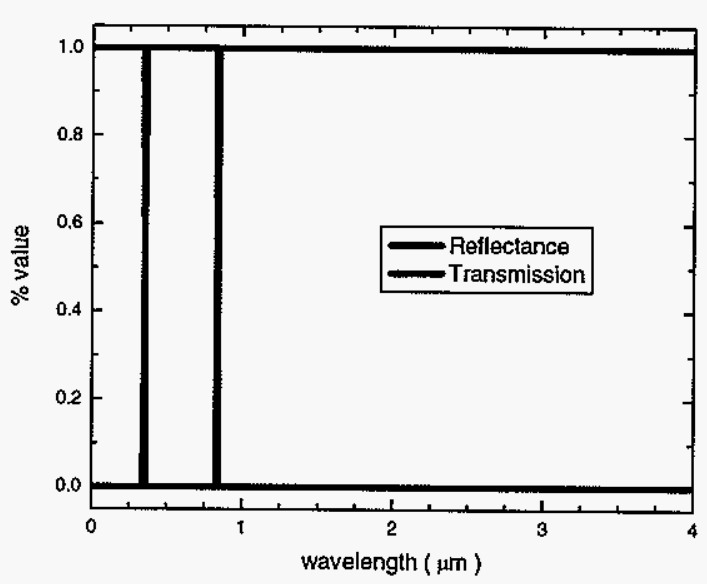

(b)

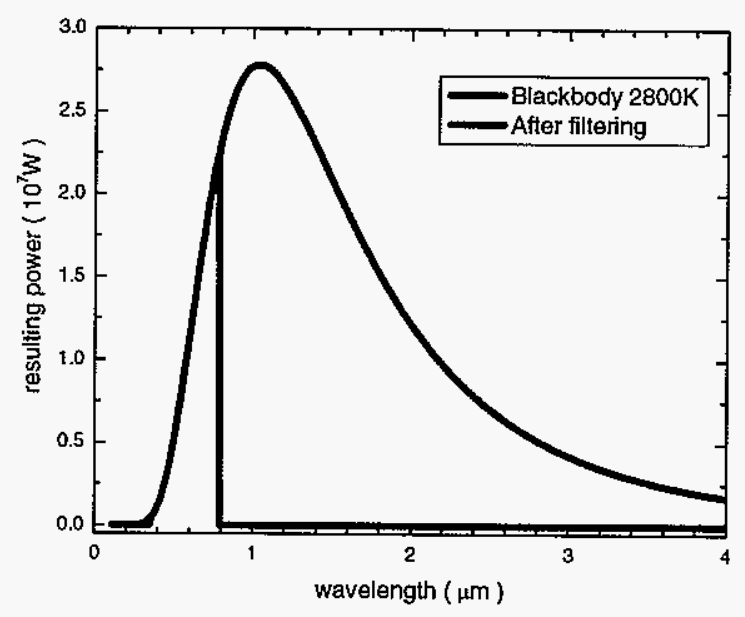

(d)

Fig. 5.3 Two ideal cases analyzed. (a) and (b) are the optical properties of $100 \%$ non-visible absorber and $100 \%$ non-visible reflector, respectively. (c) and (d) are the resulting spectra after filtering with the photonic structures represented (a) and (b), respectively. 


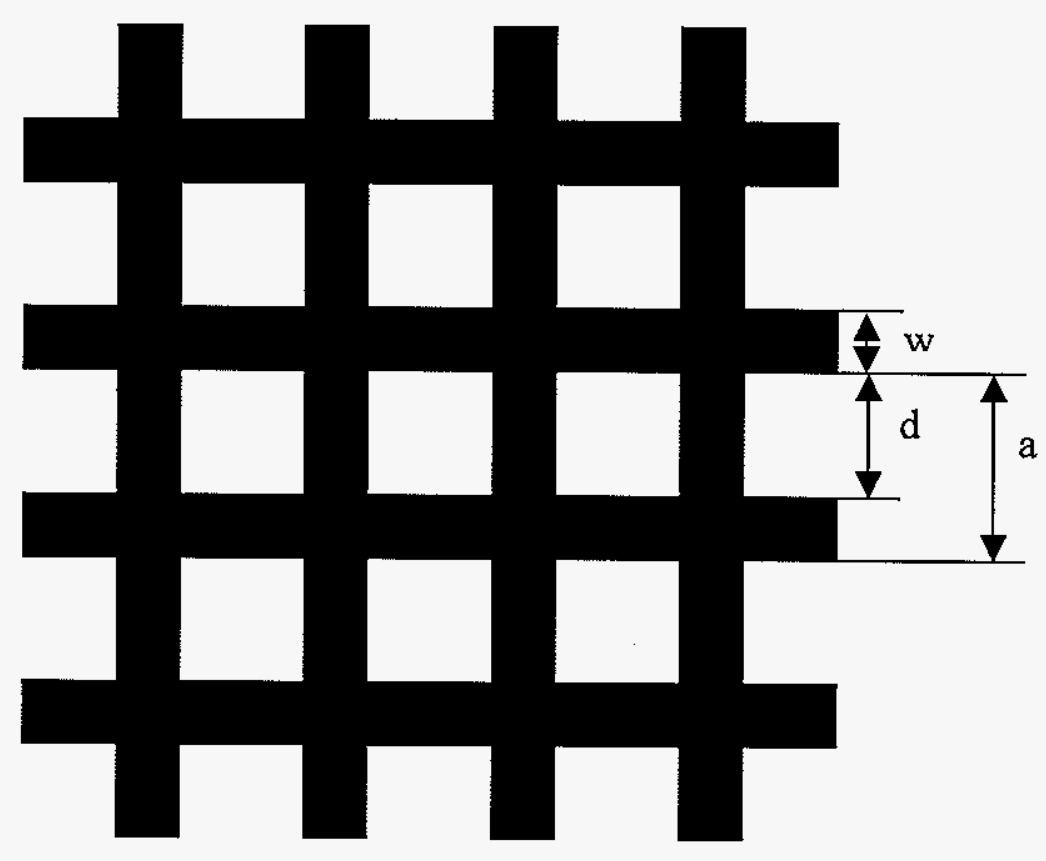

Fig. 5.4 Top view of 1-D square mesh. a is a pitch, $d$ is the size of air void and $w$ is a bar width. 


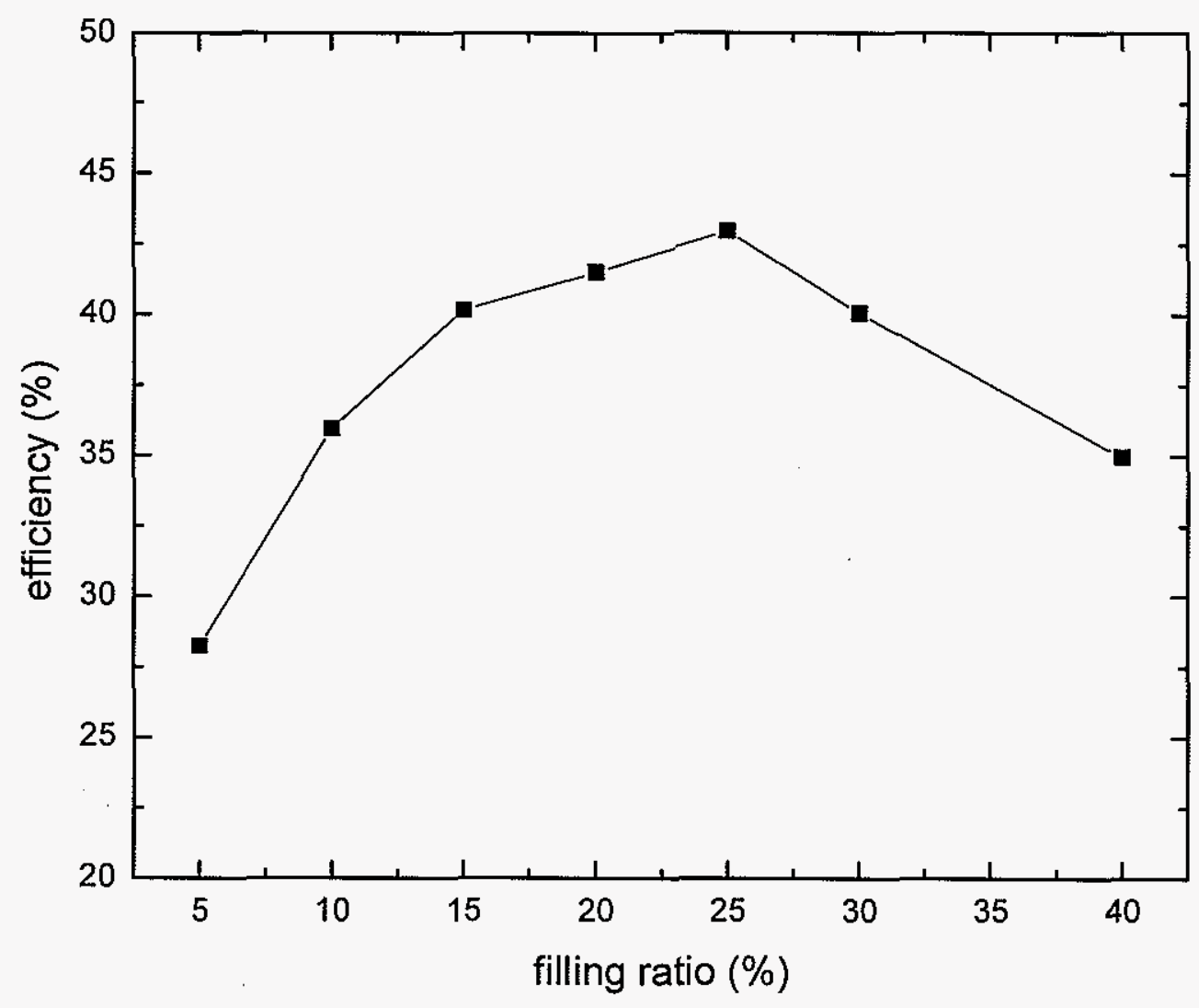

Fig. 5.5 Calculated efficiencies by changing filling fraction from $5 \%$ to $40 \%$. The thickness of the photonic structure is $500 \mathrm{~nm}$. At $25 \%$ we can see the maximum efficiency. 


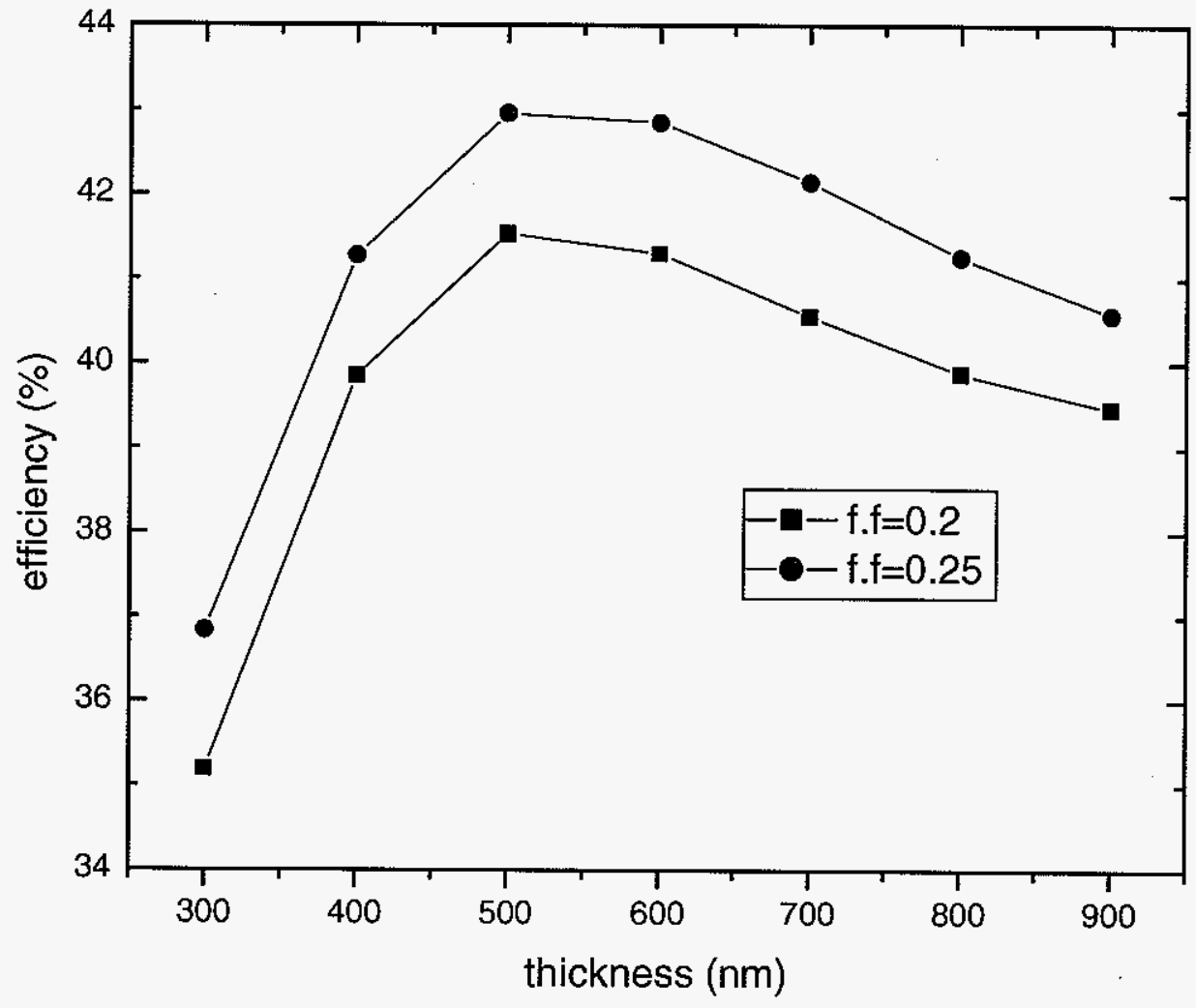

Fig. 5.6 Calculated efficiencies by changing thickness of the photonic structure. Red circles are efficiencies of filling fraction of $25 \%$ and black squares are those of $20 \%$. 


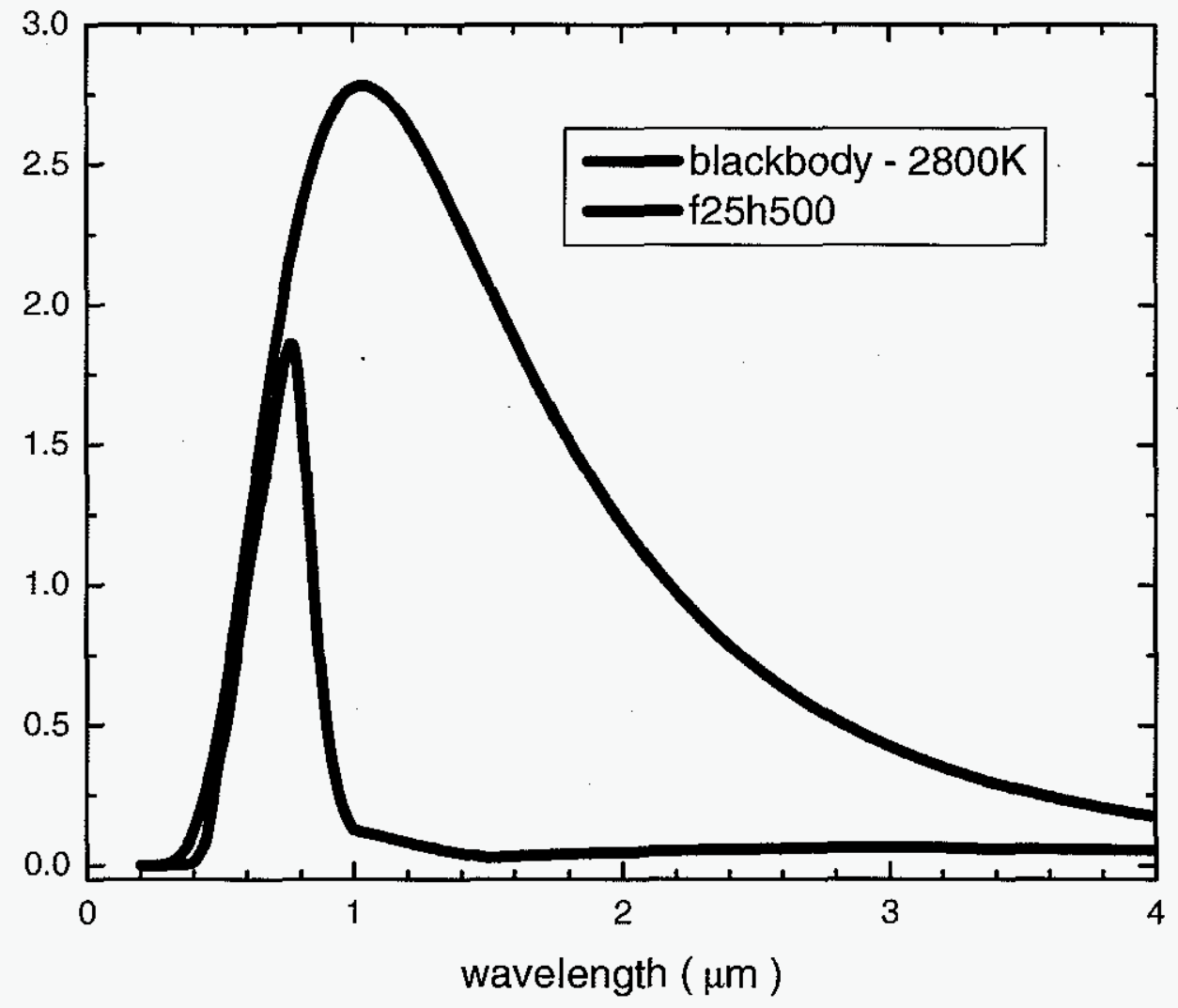

Fig. 5.7 Resulting spectrum(blue line) after filtering with the photonic structure of filling fraction $25 \%$ and thickness $500 \mathrm{~nm}$. The red line represents a blackbody spectrum at $2800 \mathrm{~K}$. 


\section{CHAPTER 6. General Conclusion}

In this thesis our main interest has been to investigate metallic photonic crystal and its applications. We explained how to solve a periodic photonic structure with transfer matrix method and when and how to use modal expansion method. Two different coating methods were introduced, modifying a photonic structure's intrinsic optical properties and rigorous calculation results are presented.

Two applications of metallic photonic structures are introduced. For thermal emitter, we showed how to design and find optimal structure. For conversion efficiency increasing filter, we calculated its efficiency and the way to design it.

We presented the relation between emitting light spectrum and absorption and showed the material and structural dependency of the absorption spectrum. By choosing a proper base material and structural parameters, we can design a selective emitter at a certain region we are interested in.

We have developed a theoretical model to analyze a blackbody filament enclosed by a metallic mesh which can increase the efficiency of converting a blackbody radiation to visible light. With this model we found that a square lattice metallic mesh enclosing a filament might increase the efficiency of incandescent lighting sources. Filling fraction and thickness dependency were examined and presented. Combining these two parameters is essential to achieve the maximum output result. 


\section{Bibliography}

[1] E. Yablonovitch, J. Opt. Soc. Am. B 10, 283 (1993)

[2] S. John and J. Wang, Phys. Rev. Lett. 642418 (1990)

[3] K. M. Ho, C. T. Chan, and C. M. Soukoulis, Phys. Rev. Lett. 653152 (1990)

[4] S. Y. Lin, J. G. Fleming, E. Chow, J. Bur, K. K. Choi, and A. Goldberg, Phys. Rev. B 62, R2243 (2000)

[5] J. G. Fleming, S. Y. Lin, I. El-Kady, R. Biswas, and K. M. Ho, Nature(London) 417, 52 (2002)

[6] Shawn-Yu Lin, J. G. Fleming, and I. El-Kady, Appl. Phys. Lett. 83, 593 (2003)

[7] Lan-Lan Lin, Zhi-Yuan Li, and Kai-Ming Ho, J. Appl. Phys. 94, 881 (2003)

[8] Zhi-Yuan Li and Kai-Ming Ho, Phys. Rev. B 67, 165104 (2003)

[9] D. Cassagne, C. Jouanin, and D. Bertho, Phys. Rev. B 53, 7134 (1996)

[10] Z. Y. Li, J. Wang, and B. Y. Gu, Phys. Rev. B 58, 3721 (1998).

[11] J. B. Pendry, J. Mod. Opt. 41, 209 (1994).

[12] P. M. Bell, J. B. Pendry, L. Marin Moreno, and A. J. Ward, Comp. Phys. Commun. 113, 306 (1995).

[13] C. T. Chan, Q. L. Yu, and K. M. Ho, Phys. Rev. B 51, 16635 (1995).

[14] N. Stefanou, V. Yannopapas, and A. Modinos, Comp. Phys. Commun. 113, 49 (1998).

[15] N. A. Nicorovici, R. C. McPhedran, and L. C. Botten, Phys. Rev. E 52, 1135 (1995). 
[16] L. Li, J. Opt. Soc. Am. A 13, 1024 (1996).

[17] J. R. Andrewartha, J. R. Fox, and I. J. Wilson, Opt. Acta 26, 69 (1979).

[18] L. C. Botten, M. S. Craig, R. C. McPhedran, J. L. Adams, and J. R. Andrewartha, Opt. Acta 28, $1087(1981)$

[19] S.Y. Lin, J.G. Fleming, and J Mereno, Appl. Phys. Lett. 83, 380, (2003).

[20] S.Y. Lin, J.G. Fleming, and I. El-Kady, Optics. Lett. 28, 1683 (2003).

[21] J.D. Joannopoulos, P.R. Villeneuve, and S. Fan, Nature 386, 143 (1997).

[22] M. Qi, E. Lidorikis, P.T. Rakich, S.G. Johnson. J.D. Joannopoulos, E.P. Ippen, and H.I.Smith, Nature 429, 538 (2004).

[23] W. Park and T. Borsa, Mat. Res. Soc. Symp. Proc. 820, R2.9.1 (2004).

[24] S. Ogawa, M. Imada, S. Yoshimoto, M. Okano, and S. Noda, Science, 305, 227 (2004).

[25] A.J. Turberfield, MRS Bulletin 26, 632 (2001).

[26] V.L. Colvin, MRS Bulletin 26, 637 (2001).

[27] W.L. Vos and A. Polman, MRS Bulletin 26, 642(2001).

[28] J.G. Fleming and S.Y. Lin, Optics Letters 24, 49 (1999).

[29] E. Ozbay, B. Temelkuran, M. Sigalas, G. Tuttle, C. M. Soukoulis, and K. M. Ho, Appl. Phys.Lett. 69, 3797 (1996).

[30] K.M. Ho, C.T. Chan, C.M. Soukoulis, R. Biswas, and M. Sigalas, Solid State Commun. 89, 413 (1994). 
[31] R. Biswas, J. Ahn, T. Lee, J. -H. Lee, Y. -S. Kim, C. -H. Kim, W. Leung, C. -H. Oh, K. Constant, and K. -M. Ho, J. Opt. Soc. Am. B 22, 2728 (2005)

[32] J.P. Singh, D.-L. Liu, D.-X. Ye, R.C. Picu, T.M. Lu, and G.C. Wang, Appl. Phys. Lett. 84, 3657 (2004)

[33] Y. -S. Kim, D. -L. Bae, H. Yang, H. -S. Shin, G.-C. Wang, J. J. Senkevich, and T.-M. Lu, J. Electrochem. Soc. 152, C89 (2005).

[34] W. Leung, H. Kang, K. Constant, D. Cann, C.-H. Kim, R. Biswas, M. M. Sigalas, and K.-M. Ho, J. Appl. Phys. 93, 5866 (2003).

[35] J. H. Lee, C. H. Kim, Y. S. Kim, K. M. Ho, K. Constant, W. Leung, and C. H. Oh, Appl. Phys. Lett. 86, 204101 (2005)

[36] Y. Miklyaev et al, Appl. Phys. Lett. 82, 1284 (2003).

[37] M. Deubel, G. Freymann, M. Wegener, S. Perereira, K. Busch, and C.M. Soukoulis, Nature Materials 3, 444 (2004).

[38] Z. Y. Li, I. El-Kady, K. M. Ho, S. Y. Lin, and J. G. Fleming, J. Appl. Phys. 93, 38 (2003)

[39] S. Y. Lin, J. G. Fleming, Z. Y. Li, I. El-Kady, R. Biswas, and K. M. Ho, J. Opt. Soc. Am. B 20, 1538 (2003)

[40] Z. Y. Li and K. M. Ho, Phys. Rev. B 67, 165104 (2003)

[41] G. von Freymanna, S. John, M. Schulz-Dobrick, E. Vekris, N. Tetreault, S. Wong, V. Kitaev, and G. A. Ozin, Appl. Phys. Lett. 84, 224 (2004) 
[42] S.Y. Lin, D.-X. Ye, T.-M. Lu, and J. Bur, Y.S. Kim and K.M. Ho (submitted)

[43] H. Raether, Surface Plasmons on Smooth and Rough Surfaces and on Gratings,

Springer, Berlin, (1988)

[44] E.D. Palik and G. Ghosh, The Electronic Handbook of Optical Constants and Thermooptic Coefficients, Elsevier Science \& Technology Books, (1999) 